\title{
Experimental Studies of Laser-induced Breakdown in Transparent Dielectrics
} C.W. Carr Ph.D. Dissertation

September 23, 2003

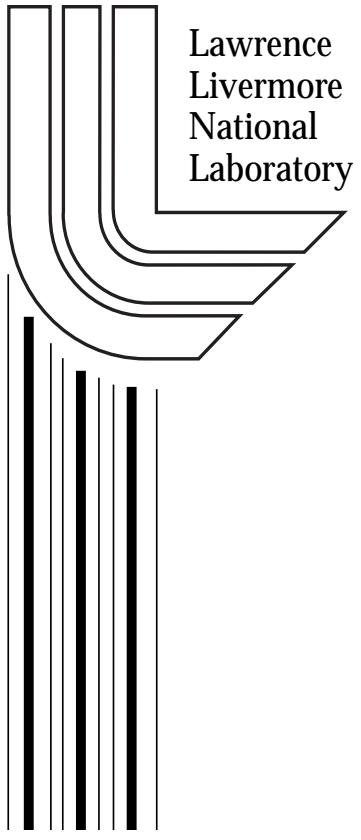




\section{Disclaimer}

This document was prepared as an account of work sponsored by an agency of the United States Government. Neither the United States Government nor the University of California nor any of their employees, makes any warranty, express or implied, or assumes any legal liability or responsibility for the accuracy, completeness, or usefulness of any information, apparatus, product, or process disclosed, or represents that its use would not infringe privately owned rights. Reference herein to any specific commercial product, process, or service by trade name, trademark, manufacturer, or otherwise, does not necessarily constitute or imply its endorsement, recommendation, or favoring by the United States Government or the University of California. The views and opinions of authors expressed herein do not necessarily state or reflect those of the United States Government or the University of California, and shall not be used for advertising or product endorsement purposes.

This work was performed under the auspices of the U. S. Department of Energy by the University of California, Lawrence Livermore National Laboratory under Contract No. W-7405-Eng-48.

This report has been reproduced directly from the best available copy.

Available electronically at http://www.doe.gov/bridge

Available for a processing fee to U.S. Department of Energy and its contractors in paper from

U.S. Department of Energy Office of Scientific and Technical Information

P.O. Box 62

Oak Ridge, TN 37831-0062

Telephone: (865) 576-8401

Facsimile: (865) 576-5728

E-mail: reports@adonis.osti.gov

Available for the sale to the public from

U.S. Department of Commerce

National Technical Information Service 5285 Port Royal Road Springfield, VA 22161

Telephone: (800) 553-6847

Facsimile: (703) 605-6900

E-mail: orders@ntis.fedworld.gov

Online ordering: http://www.ntis.gov/ordering.htm 


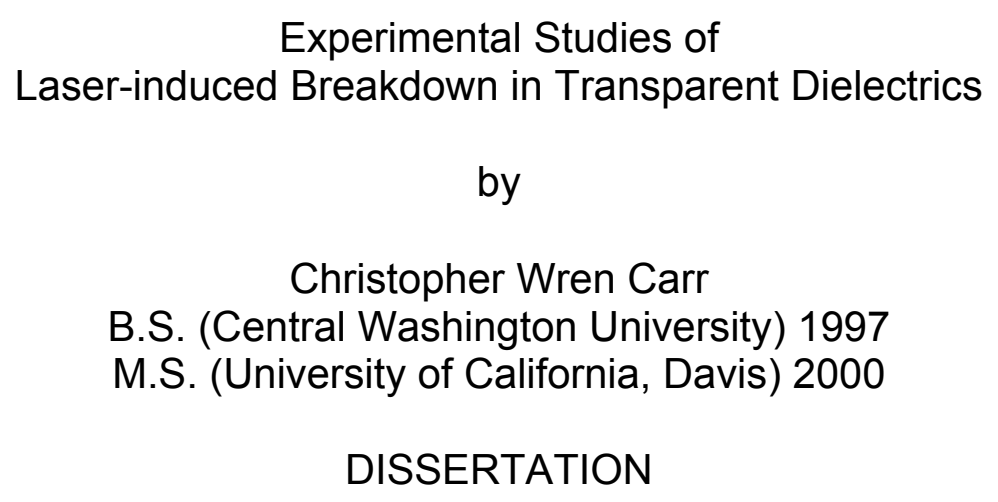

Submitted in partial satisfaction of the requirements for the degree of DOCTOR OF PHILOSOPHY

in

Physics

in the

OFFICE OF GRADUATE STUDIES

of the

UNIVERSITY OF CALIFORNIA, DAVIS

Approved:

\begin{tabular}{lc}
\hline Professor Harry Radousky, Chair & Date \\
\hline Professor Robert Shelton & Date \\
\hline Professor Shirley Chiang & Date
\end{tabular}

Committee in Charge

2003 


\author{
Abstract \\ Experimental Studies of \\ Laser-Induced Breakdown in Transparent Dielectrics \\ by \\ Christopher Wren Carr \\ Doctor of Philosophy in Physics \\ University of California, Davis \\ Professor Harry Radousky, Chair
}

The mechanisms by which transparent dielectrics damage when exposed to high power laser radiation has been of scientific and technological interest since the invention of the laser. In this work, a set of three experiments are presented which provide insight into the damage initiation mechanisms and the processes involved in laser-induced damage. Using an OPO (optical parametric oscillator) laser, we have measured the damage thresholds of deuterated potassium dihydrogen phosphate (DKDP) from the near ultraviolet into the visible. Distinct steps, whose width is of order $K_{b} T$, are observed in the damage threshold at photon energies associated with the number of photons $(3 \rightarrow 2$ or $4 \rightarrow 3$ ) needed to promote a ground state electron across the energy gap. The wavelength dependence of the damage threshold suggests that a primary mechanism for damage initiation in DKDP is a multi-photon process in which the order is reduced through excited defect state absorption. In-situ florescence microscopy, in conjunction with theoretical calculations by Liu et al., has been used to establish that hydrogen displacement defects are potentially responsible 
for the reduction in the multi-photon cross-section. During the damage process, the material absorbs energy from the laser pulse and produces an ionized region that gives rise to broadband emission. By performing a time-resolved investigation of this emission, we demonstrate both that it is blackbody in nature, and we provide the first direct measurement of the localized temperature during and following laser damage initiation for various optical materials. For excitation using nanosecond laser pulses, the plasma, when confined in the bulk, is in thermal equilibrium with the lattice. These results allow for a detailed characterization of temperature, pressure, and electron densities occurring during laser-induced damage. 


\section{Contents}

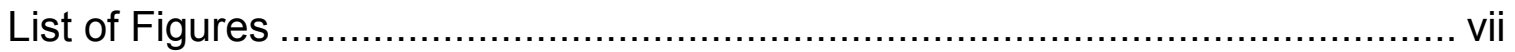

List of Tables .........................................................................................

Chapter 1: Introduction to Laser-Induced Damage in Transparent

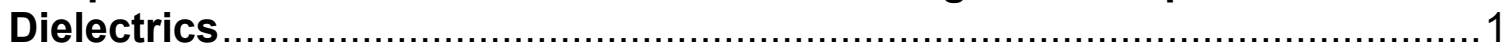

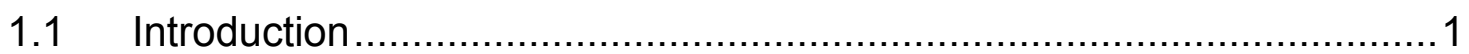

1.2 Manifestations of laser-induced damage .......................................6

1.3 The mechanisms by which laser-induced damage occur .....................9

1.4 References ...................................................................... 11

Chapter 2: Experimental Apparatus and Technique for Measuring the Wavelength Dependence of the Laser-Induced Damage Threshold ............13

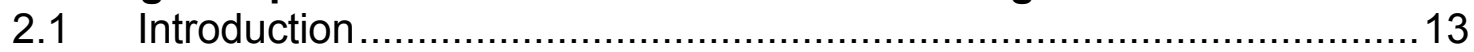

2.2 Experimental methods and techniques........................................17

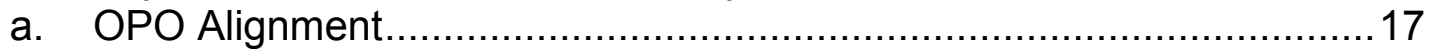

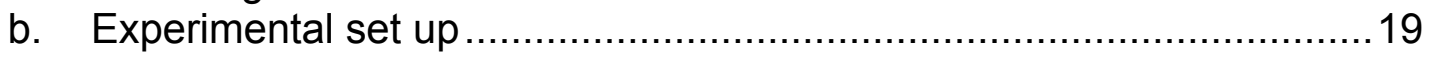

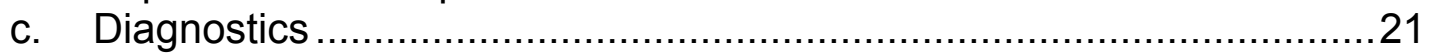

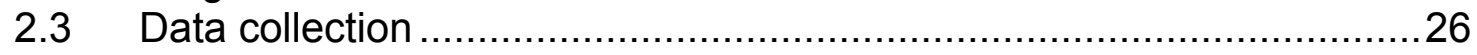

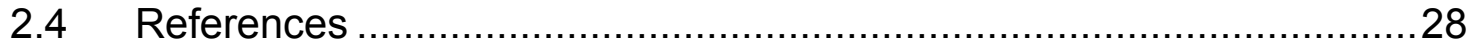

Chapter 3: Analysis of Wavelength Dependent Damage Threshold Data....29

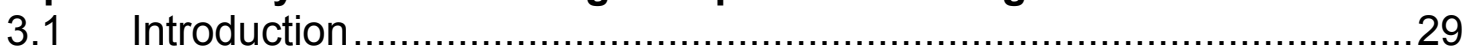

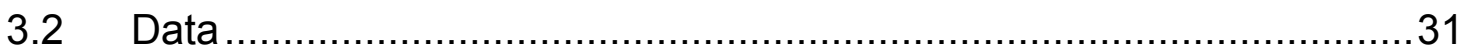

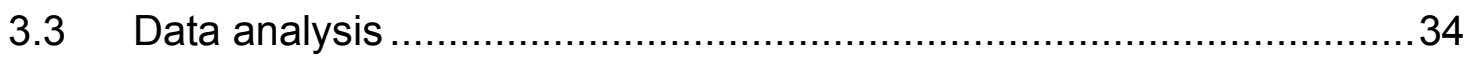

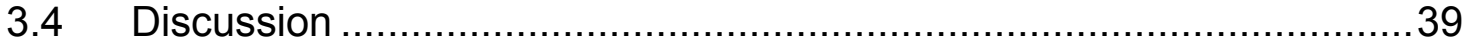

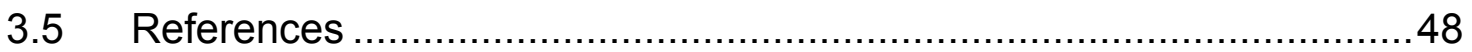

Chapter 4: Experimental Technique for In-Situ Dynamic Temperature Measurements during Laser-Induced Breakdown ..................................49

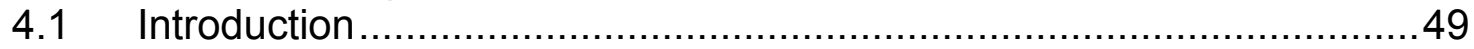

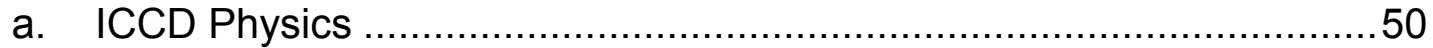

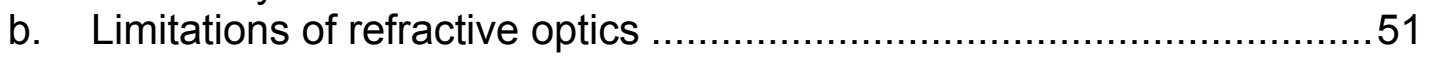

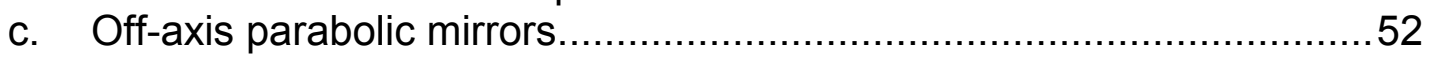

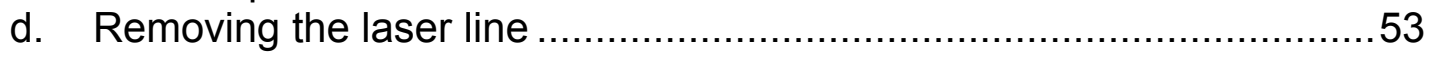

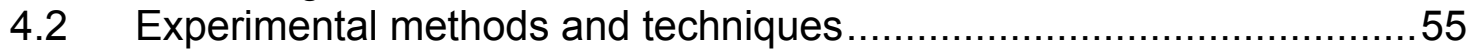

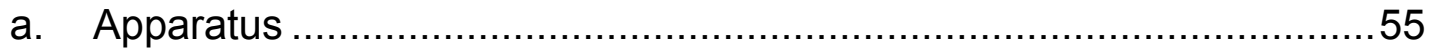

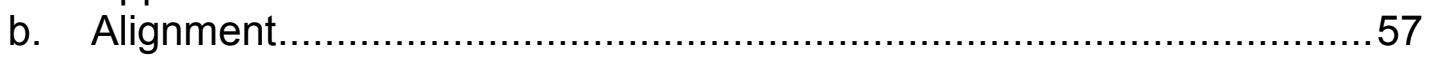

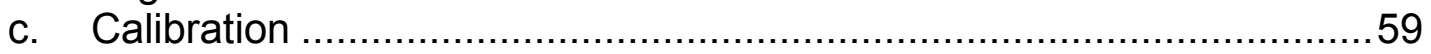

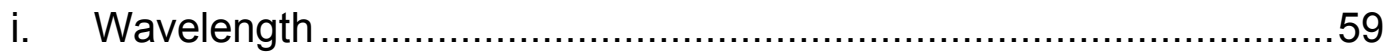

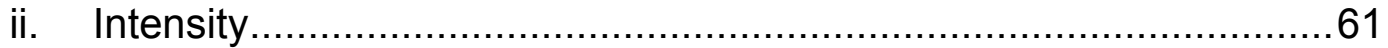

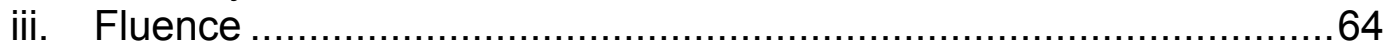

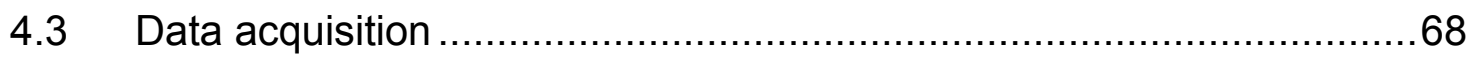

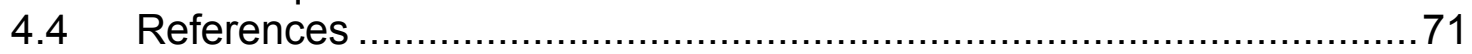

Chapter 5: Determining the Local Values of Temperature during Laser-

Induced Damage in Wide Gap Materials ................................................72

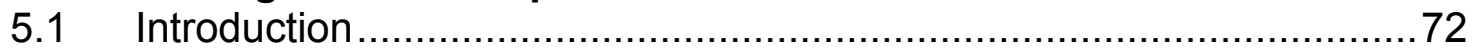

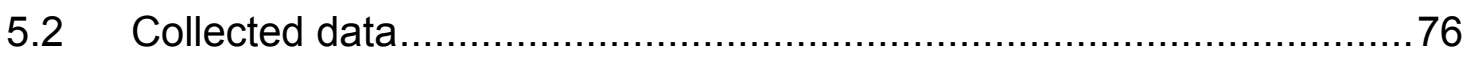

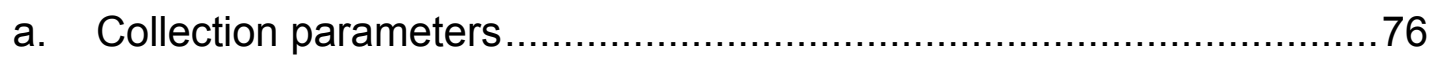




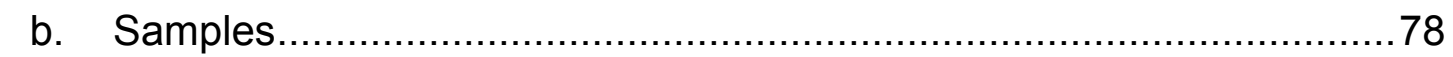

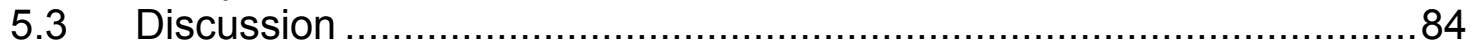

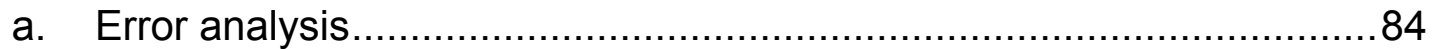

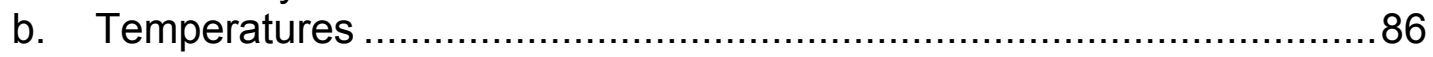

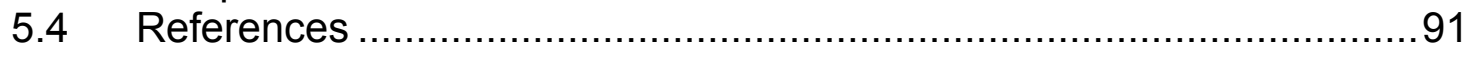

Chapter 6: Discussion and Conclusions Concerning Laser-Induced

Damage in Wide Band Gap Optical Materials .................................... 92

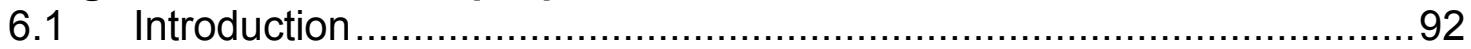

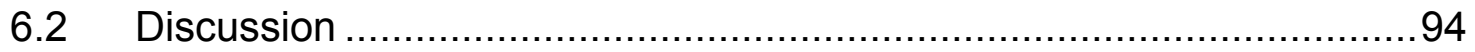

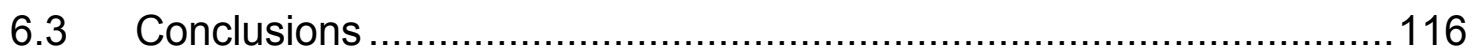

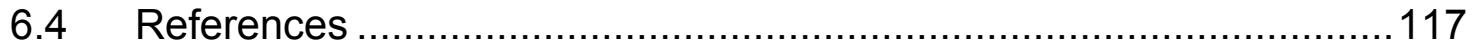




\section{List of figures}

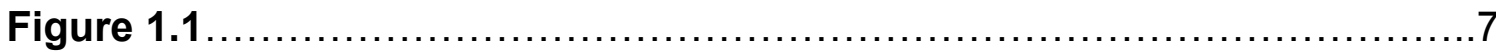

An inverted HeNe scatter image of laser damage produced in KDP by a high intensity 355-nm pulse propagating from right to left. The dark regions of the image are individual damage sites produced by the laser. The intensity distribution of the pulse is shown on the left. Note that damage is more prevalent at, but not restricted to, the peak of the pulse

Fgure 1.2 8

White light scattering image of a damage site in the bulk of a KDP crystal. The damage was produced with a two 3-ns pulses separated by 4-ns, simulating a single pulse of $10-n s$ duration.

Figure 1.3.

An STM image of laser-induced damage on the surface of $\mathrm{SiO}_{2}$. The size of the melted material is on the order of the spot size..

Figure 2.1. 16

A schematic representation of an OPO The dot next to the pump beam indicates it has ordinary polarization, while the arrows next to signal and idler indicate extraordinary polarization. The injection mirror of an OPO is transparent to the pump beam but highly reflective over a broad range of signal and/or idler wavelengths. The output coupler is only partly reflective over the signal and or idler wavelengths. Placing the converting medium between these two mirrors forms a resonant cavity to stimulate conversion. The converting medium is rotated about an axis orthogonal to the $c$ axis of the crystal and the k vector of the pump beam to angle tune the effective extraordinary index of refraction.

Figure 2.2.

A schematic representation of the ring cavity optical parametric oscillator used in these experiments. After passing through the entrance window, the pump beam is turned $90^{\circ}$ by the beam splitter. The pump beam passes through the first BBO where "walk off" occurs during conversion. The second BBO has its optical axis oriented opposite to the first so that the pump and converted beams "walk on" to one another. The unconverted pump beam will be rejected by the beamsplitter after exiting the second BBO. The converted light will pass through the second prism where the majority will be reflected out of the OPO. The remaining light will pass though the beamsplitter to stimulate conversion in the BBO during the remainder of the pump pulse. Because the optical path of the cavity formed by the two prisms is only 4 inches in length, portions of the light converted in the first part of the 3 ns pulse will make up to 9 passes in the cavity before the termination of the pump pulse. 
Figure 2.3.

1) The OPO is pumped at $266 \mathrm{~nm}$ or $355 \mathrm{~nm}$ to produce a continuous output from $310 \mathrm{~nm}$ to $650 \mathrm{~nm}$. 2) A Keplerian telescope is used to correct the wavelength dependent divergence. 3) The energy of each pulse is measured by a computer monitored thermopile energy meter. 4) Pulse energy is regulated by a pair of computer controlled polarizers which also establish a polarization purity of $1000 \mathrm{~S}: 1 \mathrm{P}$. The beam is focused by a compound lens system into the bulk of a 5) DKDP sample. 6) A cw HeNe laser is focused into the same region through the back surface of the sample.

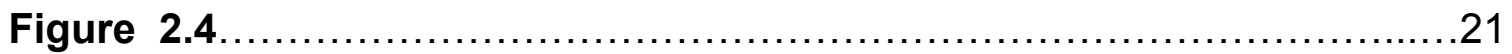

The pulse duration produced by the OPO at $340 \mathrm{~nm}, 432 \mathrm{~nm}$, and $546 \mathrm{~nm}$ as measured with a photodiode and oscilloscope.

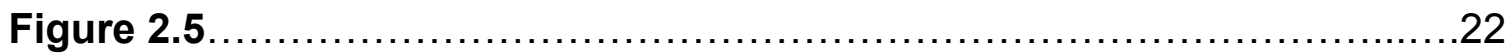

The focal spot geometry showing the beam waist for wavelengths of $532 \mathrm{~nm}$ and $333 \mathrm{~nm}$.

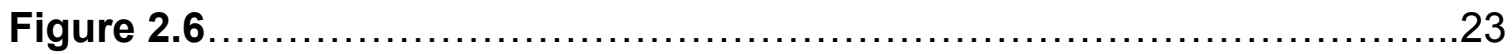

By imaging the spot size at several places along the $z$ axis and plotting the effective diameter versus the position, the beam waist can be positively identified. The Rayleigh range is seen to be on the order of $500 \mu \mathrm{m}$.

Figure 2.7 .

The beam waist was maintained at $100 \mu \mathrm{m}+/-5 \mu \mathrm{m}$ for all the wavelengths used in this experiment.

Figure 2.8.

The output of the OPO at $487.0 \mathrm{~nm}$ depicts a typical line width of $1 \mathrm{~nm}$.

Figure 2.9. 26

The CCD imaging system used for damage detection consisted of an 8 bit camera $632 \mathrm{~nm}$ band pass filter, and a focusing lens. The system was mounted on a computer controlled electromechanically actuated stage so that the focus could be adjusted by the damage acquisition software.

Figure 2.10.

The front panel of the data acquisition program. 1) The program controlled sample positioning and camera focus by commanding electromechanically actuated micrometers. 2) The damage detection camera had a spatial resolution of 15 um and could display live video or averaged images. 3) Before and after images from the damage detection camera were automatically subtracted to enhance damage detection sensitivity. 4) The energy of the laser pulse was automatically retrieved from the thermopile energy meter and combined with the beam profile data to determine the peak fluence of the shot. This information and the user determination of damage were entered into the damage probability 
vs. fluence histogram in real time. 5) Pulse energy was adjusted by a pair of polarizers. The polarizer was mounted in an electromechanically actuated rotation stage. The second polarizer was in a fixed orientation and set the polarization of the incident laser light to $1000 \mathrm{~S}: 1 \mathrm{P}$.

Figure 3.1 32

S-curves for $312.9 \mathrm{~nm}(3.96 \mathrm{eV})$ to $376.3 \mathrm{~nm}(3.29 \mathrm{eV})$ The 266-nm pumped OPO was the light source used in obtaining this data.

Figure 3.2.

S-curves for $434.1 \mathrm{~nm}(2.86 \mathrm{eV})$ to $484.5 \mathrm{~nm}(2.56 \mathrm{eV})$. The $355-\mathrm{nm}$ pumped OPO was the light source used in obtaining this data.

Figure 3.3. 33

S-curves for $486.1 \mathrm{~nm}(2.55 \mathrm{eV})$ to $532.5 \mathrm{~nm}(2.32 \mathrm{eV})$. The $355-\mathrm{nm}$ pumped OPO was the light source used in obtaining this data.

Figure 3.4. 34

The fluences at which each of the 7 wavelengths between $312.9 \mathrm{~nm}$ and 376.3 $\mathrm{nm}$ produced damage $50 \%$ of the time is seen by rescaling the vertical axis of figure 3.3.

Figure 3.5. 35

The fluences at which each of the 7 wavelengths between $434.1 \mathrm{~nm}$ and 484.5 $\mathrm{nm}$ produced damage $50 \%$ of the time is seen by rescaling the vertical axis of figure 3.4.

Figure 3.6. 35

The fluences at which each of the 7 wavelengths between $486.1 \mathrm{~nm}$ and 532.5 $\mathrm{nm}$ produced damage $50 \%$ of the time is seen by rescaling the vertical axis of figure 3.5.

Figure 3.7. 36 The wavelength dependent damage threshold is obtained by plotting wavelength vs. the fluence at which the sample damaged $50 \%$ of the time at that wavelength.

Figure 3.8. 37

Damage probability for $24 \mathrm{~J} / \mathrm{cm}^{2}$ for wavelengths near the $487 \mathrm{~nm}$ step. The damage probabilities for $486.1 \mathrm{~nm}$ and $486.9 \mathrm{~nm}$ are both unity.

Figure 3.9. 37

Damage probability for $7.5 \mathrm{~J} / \mathrm{cm}^{2}$ for wavelengths near the $319 \mathrm{~nm}$ step. The damage probabilities for $333.2 \mathrm{~nm}, 349.9 \mathrm{~nm}$, and $376.3 \mathrm{~nm}$ are al zero. 
Figure 3.10

Damage probability at fixed fluences plotted vs. photon energy difference from each damage step $\left(\varepsilon_{\text {photon }}-\varepsilon_{c}\right)$. The width of the transition is approximately $\mathrm{K}_{\mathrm{b}} \mathrm{T}$.

Figure 3.11 39

Normalized transmission through a 1-cm thick piece of DKDP.

Figure 3.12.

Light scattering image of DKDP material modified by a 355-nm 3-ns laser pulse. The laser pulse propagated from left to right. The fluence distribution of the beam was Gaussian in the vertical direction. The image shows that the damage is not restricted to the peak fluence, but distributed through the beam.

Figure 3.13

Individual photons with energies less than the band gap may be absorbed if electrons in the valence band can be excited into defect states within the band gap. This mechanism would allow photons to be absorbed in series rather than simultaneously, reducing the intensity (photon density) necessary to excite an electron from valence to conduction bands.

Figure 4.1. 50

A schematic of a Roper Scientific ICCD camera similar to the one used in these experiments. A CCD array records the intensified image.

Figure 4.2

The focal spots produced by a fused silica lens and an off-axis parabolic mirror when focusing a broadband light source. Because the focal length of the lens is slightly different for each wavelength the tightest focal spot is nearly double that produced by the mirror.

Figure 4.3 53

Transmission spectrum of a 355-nm notch filter. The spectrum shows that the filter will block a 355-nm laser line, but only at the cost of losing the light below $300 \mathrm{~nm}$.

Figure 4.4 .56

A schematic diagram of the apparatus. A pump beam is introduced into the system by a small turning mirror. The pump beam is focused by an off-axis parabolic mirror into the bulk of a sample. The plasma produced emits broadband radiation of which a fraction is collected by the off-axis parabolic mirror. This emission is collimated by the mirror into a 3 " beam counterpropagating with the pump beam. Because the emission beam overfills the small turning mirror, only $16 \%$ of the emission is reflected out of the beam. The remaining emission continues past the turning mirror and is incident on a second off-axis parabolic mirror. This second mirror focuses the emission beam onto the slit of a spectrometer. 
Figure 4.5

Atomic line spectrum from a HgNe lamp. A 260-nm long pass filter is used to attenuate the $250 \mathrm{~nm}$ line.

Figure 4.6.

The spectra produced by the QTH (blackbody) lamp and the $D_{2}$ arc lamp. The UV end of the D2 lamp is clipped by a $225-\mathrm{nm}$ long pass filter.

Figure 4.7. 63

The calibration curve which converts the counts collected by the ICCD into absolute units. The curve represents the combined effects of non uniform transmission from optics, quantum efficiency of the ICCD, and f number of the collecting mirror.

Figure 4.8. .64

Schematic representation of a knife edge measurement. Incident and transmitted energy is monitored, with the difference attributed to obscuration by the blade. Plotting the derivative of transmitted energy vs. blade position indicates the profile of the beam where it was cut by the blade.

Figure 4.9 a 65

Transmission vs. blade position data taken during a knife edge measurement.

Figure $4.9 \mathrm{~b}$ 66 The derivative of the data in figure 4.9a. The data show the profile of the beam in one dimension. A Gaussian fit gives the spot size as $46.3 \mu \mathrm{m}$.

Figure 4.10.

The front panel of the automated knife edge measurement program.

Figure 4.11

The individual symbols are representative points from the calibrated spectra from DKDP. The solid curves through the data are the best fits to a Planckian distribution (equation 4.2). The legend indicates the excitation wavelength and the delay time for which the data were acquired. The $2 \omega 105 \mathrm{~ns}$ data and fit has been multiplied by a factor of 10 to enhance its visibility.

Figure 4.12. .70

Fitted plasma temperatures for fused silica data. The temperatures plotted as a function of delay time as extracted from the fit to equation 1 of the time-gated emission spectra under 1064-nm, 532-nm, and 355-nm excitation. A schematic representation of the 1064-nm pulse is shown in arbitrary units. 
Figure 5.1.

The image on the left shows the emission produced by the plasma accompanying the laser-induced breakdown at each of three sites. The damage was induced by a pulsed $355 \mathrm{~nm}$ laser focused with a cylindrical lens. The damaging beam overfills the image and is propagating left to right. The image was acquired with a CCD camera equipped with a long pass filter to remove both scattered laser light and Raman shifted light. On the right, a scattering image taken using a cw HeNe laser for illumination 10 seconds after the 355-nm pulse is terminated. Sites in the right hand image are visible because the host matrix has been permanently modified by the breakdown event (laser damage). Discontinuity in material composition scatters the HeNe light by 90 degrees (into the CCD camera). The damage sites evident in the right hand image are consistent with the observation that whenever laser damage occurs it is accompanied by plasma emission.

Figure 5.2.

From Gorbunov, A.V. and M. Maksimuk, Fiz Tverd Tela, 1994. 36(5): p. 1429-36 The spectra of plasma emission accompanying breakdown in $\mathrm{NaCl}$ crystals.

Figure 5.3. .75

From Bandis et al. / Applied Surface Science 197-198 (2002) 100-106 "Spectrum of the visible radiation emitted from a $\mathrm{NaNO}_{3}$ bulk emission sites upon exposure to1064 $\mathrm{nm}$ laser radiation with fluence near the single pulse damage threshold. The absence of emission at wavelengths lower than $350 \mathrm{~nm}$ is consistent with absorption from the crystal surrounding the emission sites. Also note the absence of any sharp peaks due to atomic emission"

Figure 5.4.

Uncalibrated data taken in DKDP $30 \mathrm{~ns}$ after the peak of the 355-nm damaging pulse. The dip in the spectra is produced by the obstruction used to attenuate the 355-nm laser line. Despite the obstruction some laser light still incidents on the ICCD, precluding image acquisition during and immediately after the pulse.

Figure 5.5.

Emission spectra accompanying laser-induced breakdown in LiF from 1064-nm pulses $3 \mathrm{~ns}$ and $6 \mathrm{~ns}$ after the peak of the pulse and $100 \mathrm{~ns}$ after the peak of a $355-\mathrm{nm}$ pulse. The solid symbols are the representative experimental data (only every $15^{\text {th }}$ point is shown for clarity). The solid lines are the fits to the Planckian distribution. The 3- $\omega$ 100-ns data and fit have been multiplied by 10 to enhance their visibility. 
Figure 5.6.

Emission spectra accompanying laser-induced breakdown in $\mathrm{SiO}_{2}$ from 1064-nm pulses $2 \mathrm{~ns}$ and $8 \mathrm{~ns}$ after the peak of the pulse and $22 \mathrm{~ns}$ after the peak of a $355-\mathrm{nm}$ pulse. The solid symbols are the representative experimental data (only every $15^{\text {th }}$ point is shown for clarity). The solid lines are the fits to the Planckian distribution.

Figure 5.7. .80 Emission spectra accompanying laser-induced breakdown in $\mathrm{Al}_{2} \mathrm{O}_{3}$ from 1064$\mathrm{nm}$ pulses $3 \mathrm{~ns}$ and $30 \mathrm{~ns}$ after the peak of the pulse and $15 \mathrm{~ns}$ after the peak of a 355-nm pulse. The solid symbols are the representative experimental data (only every $15^{\text {th }}$ point is shown for clarity). The solid lines are the fits to the Planckian distribution. The 1- $\omega$ 30-ns and 3- $\omega$ 15-ns data and their fits have been multiplied by a factor of 5 to enhance their visibility.

Figure 5.8.

Emission spectra accompanying laser-induced breakdown in $\mathrm{CaF}_{2}$ from 1064-nm pulses $5 \mathrm{~ns}$ and $510 \mathrm{~ns}$ after the peak of the pulse and $20 \mathrm{~ns}$ after the peak of a $355-\mathrm{nm}$ pulse. The solid symbols are the representative experimental data (only every $15^{\text {th }}$ point is shown for clarity). The solid lines are the fits to the Planckian distribution. The 1- $\omega$ 510-ns and 3- $\omega$ 20-ns data and fits have been multiplied by 5 and 2 , respectively, to enhance their visibility.

Figure 5.9.

Emission spectra accompanying laser-induced breakdown in CsI from 1064-nm pulses $3 \mathrm{~ns}, 12 \mathrm{~ns}$, and $100 \mathrm{~ns}$ after the peak of the pulse. The cut off in the data at $\sim 325 \mathrm{~nm}$ is due to the absorption edge of Csl. The solid symbols are the representative experimental data (only every $15^{\text {th }}$ point is shown for clarity). The solid lines are the fits to the Planckian distribution with the data below $350 \mathrm{~nm}$ ignored during fitting. The 1- $\omega 100-n s$ data and fit have been multiplied by 2 to enhance their visibility

Figure 5.10 .83

Emission spectra accompanying laser-induced breakdown in DKDP from 1064$\mathrm{nm}, 355-\mathrm{nm}$ and 532-nm pulses $3 \mathrm{~ns}, 30 \mathrm{~ns}$, and $105 \mathrm{~ns}$, respectively, after the peak of the pulse. The solid symbols are the representative experimental data (only every $15^{\text {th }}$ point is shown for clarity). The solid lines are the fits to the Planckian distribution. The 2- $\omega 105-n s$ data and fit have been multiplied by 10 to enhance their visibility 
Figure 5.11

The solid dots are a calibrated data set smoothed with a Stineman function. The error bars encompass the unsmoothed data points to the $2 \sigma$ level. The central solid line indicates the best fit Planckian distribution (with a temperature of $\sim 4700$ $\mathrm{K})$. The 3 lines above and below the best fit show the fits where the temperatures are forced to vary from best fit value while the magnitude (effective emissivity) is allowed to float.

Figure 5.12

Temperature vs. time data taken in $\mathrm{SiO}_{2}$ for 355-nm, 532-nm, and 1064-nm experiments. The temperatures obtained from the various pump wavelengths are indistinguishable within the error of the measurement. A schematic representation of the 1064-nm laser intensity is shown in arbitrary units.

Figure 5.13

Temperature vs. time data for $\mathrm{Al}_{2} \mathrm{O}_{3}$ taken with 1064-nm pump radiation. The temperature is seen to fall rapidly after the termination of the laser pulse and then decreases at a slower rate.

Figure 5.14

Temperature vs. time data for $\mathrm{CaF}_{2}$ taken with 1064-nm pump radiation. The temperature is seen to fall rapidly after the termination of the laser pulse and then decreases at a slower rate.

Figure 5.15 88

Temperature vs. time data for CsI taken with 1064-nm pump radiation. The temperature is seen to fall rapidly after the termination of the laser pulse and then decreases at a slower rate.

Figure 5.16. 88

Temperature vs. time data for DKDP taken with 1064-nm pump radiation. The temperature is seen to fall rapidly after the termination of the laser pulse and then decreases at a slower rate.

Figure 5.17. 89

Temperature vs. time data for LiF taken with 1064-nm pump radiation. The temperature is seen to fall rapidly after the termination of the laser pulse and then decreases at a slower rate.

Figure 5.18. 89

Peak temperature of the materials is plotted vs. material band gap. The straight line is a guide to the eye which suggests a linear relationship between band gap and peak temperature. The reduced temperature observed in sapphire is consistent with the large thermal conductivity of the material. 
Figure 6.1.

The wavelength dependence of the damage threshold predicted by the impact ionization and multi-photon models.

Figure 6.2.

The wavelength dependence of the laser-induced breakdown threshold predicted by the gray particle model, assuming a uniform size distribution of inclusions.

Figure 6.3. .98

The $50 \%$ damage threshold as a function of photon energy.

Figure 6.4. 100

A schematic representation of how multi-photon absorption can lead to the steps observed in the damage threshold in figure 3.3. A band gap value of $7.5 \mathrm{eV}$ is used for simplicity.

Figure 6.5. 101

Six types of defects calculated to be present in KDP. In DKDP, the Hydrogen would be replaced with deuterium. "Relaxed atomic configurations and reactions for interstitial $\mathrm{H}$ for the neutral (a), negative (b) and positive (c) charged states. Also we show the relaxed atomic configurations for the $\mathrm{H}$ vacancy in the neutral (d), negative (e) and positive (f) charged states." - Liu et al.

Figure 6.6. 102

The density of states calculated by Liu et al. "...pure KDP (a), KDP with an interstitial $\mathrm{H}$ in the neutral state (b), and KDP with a $\mathrm{H}$ vacancy in the positive charged state (c)".

Figure 6.7 a \& b. 103

a) Fluorescing centers observed in KDP. b) High resolution image of the fluorescing centers from.

Figure 6.8. 104

a) The original image of fluorescing centers before exposure to $355-\mathrm{nm}$ 3-ns pulsed radiation. b) A difference image showing (white dots) clusters which have disappeared and (black dots) new clusters which have appeared after illumination with pulsed radiation.

Figure 6.9 104

The density of fluorescing centers after illumination with between 0 and $10^{4} 355$ $\mathrm{nm}$ 3-ns pulses for four orders of magnitude in fluence. 
Figure 6.10

A schematic representation of the processes involved in the proposed model. Vectors a. b. and c. represent the rates at which defects are dispersed into the background by removed clusters, incorporated into clusters from the background, and rendered inactive, respectively.

Figure 6.11.

The populations of FCs predicted by the model outlined in the text under three different simulated fluences. The qualitative match to the experimental data is exact.

Figure 6.12.

109

Spectra obtained from $\mathrm{SiO}_{2}$ during experiments with identical geometry, but pumped with a 1064-nm 5-ns pulse or 820 -nm 150-fs pulse.

Figure 6.13

Plot by A. Rubenchik et al. from SESAME Report on the Los Alamos Equation of State Library. (1983) The equation of state enables pressure and energy density estimates from the temperature measurements.

Figure 6.14

The partition of energy dissipation for the damage site. 


\section{List of Tables}

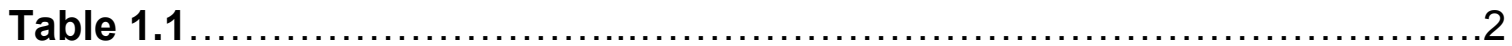

Laser-induced breakdown mechanisms and where they typically dominate the intrinsic bulk damage. The mechanisms that dominate in any situation will depend on pulse duration, laser wavelength, and the properties of the material. The typical intensities also specifically exclude effects caused by non-intrinsic properties of materials such as cracks, pores, or impurities.

Table 2.1

Polarizations for type I and type II phase matching in uniaxial crystals (such as the beta barium borate used in these experiments).

Table 3.1

Potential impurities in DKDP with their band gaps and the concentration of there constituent elements in the solution used to grow the crystal. * $\mathrm{Cd}$ was not detected.

Table 5.1

$\mathrm{T}_{3}, \mathrm{~T}_{20}$, and $\mathrm{T}_{200}$ are the temperatures of each material at delays of $3 \mathrm{~ns}, 20 \mathrm{~ns}$, and $100 \mathrm{~ns}$ respectively. The room temperature values of band gap $\left(E_{g}\right)$ and thermal conductivity $(\sigma)$ for each material are also listed. *Signal was below the experimental detection limit.

Table 6.1. 111

The energies necessary to singly ionize the relevant elements in $\mathrm{SiO}_{2}$ and DKDP. 


\section{Chapter 1}

\section{Introduction to Laser-Induced Damage in Transparent}

\section{Dielectrics}

\subsection{Introduction}

One of the hallmarks of laser-induced optical breakdown is a broadband flash of light. In 1962, this effect was first observed in air, and then in a transparent dielectric in $1964[1,2]$. The emission is now known to be produced by laser generated plasma. High intensity laser light has since been demonstrated to be capable of producing breakdown in any transparent or opaque material. When optical breakdown occurs in transparent dielectrics, the region affected is permanently modified. Although this can be a valuable feature if the desired application is laser machining or data storage, it is decidedly undesirable when this material modification occurs within optical systems such 
as lasers or telecommunication equipment [3-14]. For many optical systems, such as frequency conversion, which depend on non-linear effects, materials capable of accepting high energy densities of laser light without damage is the limiting factor for the efficiency of the devices [15-18].

Intrinsic damage and extrinsic damage are two distinct categories of laserinduced damage. Intrinsic damage occurs when a pure, or ideal, material breaks down by mechanisms which may be entirely attributed to the bulk properties. The mechanisms of intrinsic damage are generally believed to be well understood from femtosecond pulses to $\mathrm{cw}$ radiation $[6,9,12,14,19-43]$. The mechanisms of intrinsic damage can be subdivided further depending on the duration of the laser pulse (see table 1).

\begin{tabular}{|l|l|l|l|}
\hline Mechanism & Dominant Regime & \multicolumn{1}{|c|}{$\begin{array}{l}\text { Governing Material } \\
\text { Property }\end{array}$} & $\begin{array}{l}\text { Typical } \\
\text { Intensity } \\
\left(\mathrm{W} / \mathrm{cm}^{2}\right)\end{array}$ \\
\hline $\begin{array}{l}\text { Linear } \\
\text { absorption }\end{array}$ & $\begin{array}{l}\mu \mathrm{s} \text { pulsed to cw } \\
\text { lasers }\end{array}$ & Linear absorption & $<10^{9}$ \\
\hline $\begin{array}{l}\text { Electron impact } \\
\text { ionization }\end{array}$ & $\begin{array}{l}0.1 \mathrm{~ns} \text { to } \mu \mathrm{s} \text { pulsed } \\
\text { lasers }\end{array}$ & $\begin{array}{l}\text { Band gap and electron- } \\
\text { lattice collision time }\end{array}$ & $10^{9}$ to $10^{12}$ \\
\hline $\begin{array}{l}\text { Multi-photon } \\
\text { ionization }\end{array}$ & $\begin{array}{l}\text { fs and ps pulsed } \\
\text { lasers }\end{array}$ & Band gap & $10^{12}$ to $10^{16}$ \\
\hline
\end{tabular}

Table 1.1

Laser-induced breakdown mechanisms and where they typically dominate the intrinsic bulk damage. The mechanisms that dominate in any situation will depend on pulse duration, laser wavelength, and the properties of the material. The typical intensities also specifically exclude effects caused by non-intrinsic properties of materials such as cracks, pores, or impurities.

The linear absorption mechanism is perhaps the simplest, as it consists of individual photons being absorbed by a material that is less than perfectly transparent at the wavelength in question. Laser-induced damage occurs when the lattice absorbs sufficient energy to produce melting and cracking. Linear 
absorption is associated with high "collateral damage," meaning that material modification is not restricted only to the immediate focus of the laser.

For shorter (ns) pulses, the linear absorption mechanism is not fast enough to generate sufficient power, and thereby produce damage. At these pulse durations, the electron impact ionization mechanism (also called electron avalanche) will dominate. Impact ionization requires an initial source of "seed" electrons to proceed. This initial electron population can arise from sources such as thermally excited 'hot' electrons from the high energy end of the MaxwellBoltzmann distribution, or by multi-photon ionization. Regardless of the electrons' origins, they absorb energy until their kinetic energy is large enough to liberate additional electrons. Hence, during each 'generation', the number of free electrons will double. This continues until a plasma with an electron density $\left(n_{e}\right)$ close to the critical density $\left(n_{c}\right)$ is achieved. The critical density is wavelength $(\lambda)$ dependent and can be determined from equation 1.1 (which is derived from the definition of the plasma frequency)

$$
n_{c}=\left(\frac{2 \cdot \pi \cdot c}{\lambda} / 5.6 \times 10^{4} s\right)^{2} \mathrm{~cm}^{-3}
$$

Once the plasma becomes opaque, (when $n_{e} \sim n_{c}$ ) energy can be rapidly absorbed from the laser pulse. The efficiency of the mechanism is governed by the impact ionization rate, equation 1.2

$$
\frac{d n_{e}}{d t}=\gamma(E) n_{e}
$$


where $\gamma$ is the avalanche ionization coefficient. The avalanche ionization coefficient is generally only large enough for the mechanism to be significant if the ratio of band gap to photon energy is 5 or greater.

In the case that $E_{g} / h \omega<4$ or pulses are in the fs and ps regime, intrinsic damage is generally dominated by multi-photon generation of free electrons. Multi-photon ionization is when multiple photons are simultaneously absorbed by a single electron. If the sum of the photon energies is larger than the material band gap, the electron will be ionized. The number of photons necessary to bridge the gap is known as the order $(\mathrm{m})$ of the multi-photon ionization. The rate at which electrons are ionized in this manner is given by equation 1.3 [33],

$$
\frac{d n_{e}}{d t}=N \sigma^{(m)} F^{m}
$$

$\mathrm{N}, \sigma, \mathrm{m}$, and $\mathrm{F}$ are the density of active ions, the multi-photon cross-section, the order, and the photon flux density, respectively. It is important to note that $\sigma^{(m)}$ is the multi-photon cross-section of order $m$, not a cross-section to the $\mathrm{m}^{\text {th }}$ power. The value of the $\sigma^{(m)}$ is material dependent, but is largely determined by the value of $m$. As in the case with impact ionization, once $n_{c}$ is reached, energy can be rapidly absorbed by the opaque plasma.

Despite the knowledge that has been gained concerning the intrinsic damage initiation process, the phenomenon of extrinsic laser-induced damage is still not well understood $[20,21,30,33,39,40,43-54]$. Answering the basic scientific question of why the observed damage thresholds for nanosecond pulses in transparent dielectrics are an order of magnitude below the intrinsic values also has practical implications for improving the performance in 
technologically important materials [55]. This dissertation is dedicated to furthering the understanding of the extrinsic laser-induced damage process. With this aim in mind, unless explicitly stated, all future references to laserinduced damage will be understood to be addressing damage which is extrinsic in nature. 


\subsection{Manifestations of laser-induced damage}

Although laser-induced damage is observed for all wavelengths and pulse durations, it is manifested differently depending on the duration of the laser pulse. Damage from femtosecond pulses is well understood to originate from a multi-photon process, even in relatively impure samples $[4,5,46,47,56]$. In this process electrons absorb many photons simultaneously and transition from the valence band to the conduction band. The number of photons which must be simultaneously absorbed (referred to as the order of the process) is the ratio of band gap energy to photon energy. The order of a multi-photon absorption in a wide gap material for visible light is generally 4 or larger. The non-linear nature of the absorption is reflected in the sharp damage threshold and highly localized damage observed with femtosecond lasers.

This is in stark contrast to laser-induced damage observed from nanosecond lasers. Damage produced by nanosecond lasers is much more sensitive to variations in the material, making it probabilistic in nature. The likelihood of a laser pulse producing damage in a material is dependent on the laser fluence, but it is also dependent on the volume of material illuminated [5760]. The larger volume of material illuminated, the larger the chance that a section of material which is particularly susceptible to damage is included.

From the morphology of ns damage, several things can be ascertained. Because ns damage is dispersed throughout an illuminated volume in discrete

localized centers (see figure 1.1), it is obvious that the damage depends on a factor extrinsic to the transparent dielectric[59, 61]. 


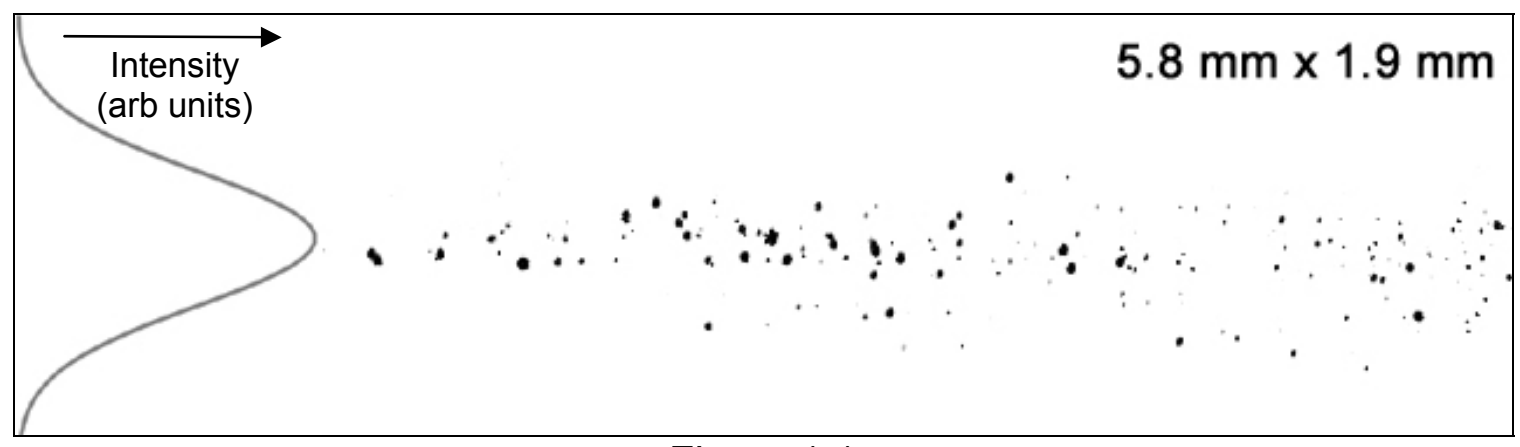

Figure 1.1

An inverted HeNe scatter image of laser damage produced in KDP by a high intensity $355-\mathrm{nm}$ pulse propagating from right to left. The dark regions of the image are individual damage sites produced by the laser. The intensity distribution of the pulse is shown on the left. Note that damage is more prevalent at, but not restricted to, the peak of the pulse.

A high resolution light scattering image of an individual damage site can be seen

in figure 1.2. The bright region in the center of the damage site is believed to have been molten during the pulse while the majority of the damage was caused by fracture around the site. 


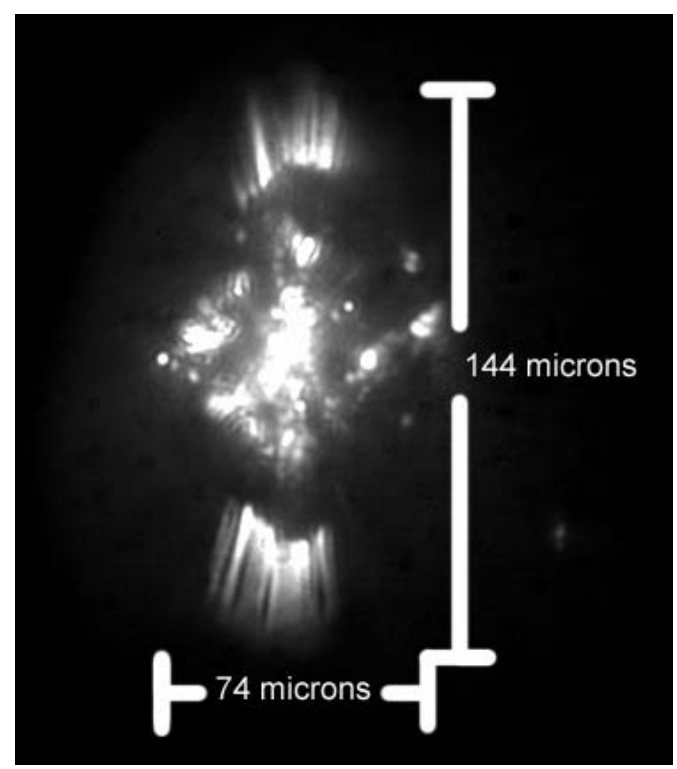

Figure 1.2

White light scattering image of a damage site in the bulk of a KDP crystal. The damage was produced with a two 3-ns pulses separated by 4-ns, simulating a single pulse of 10 -ns duration.

The STM image of laser-induced damage on the surface of a sample shown in figure 1.3 clearly shows both the melted and fractured regions.

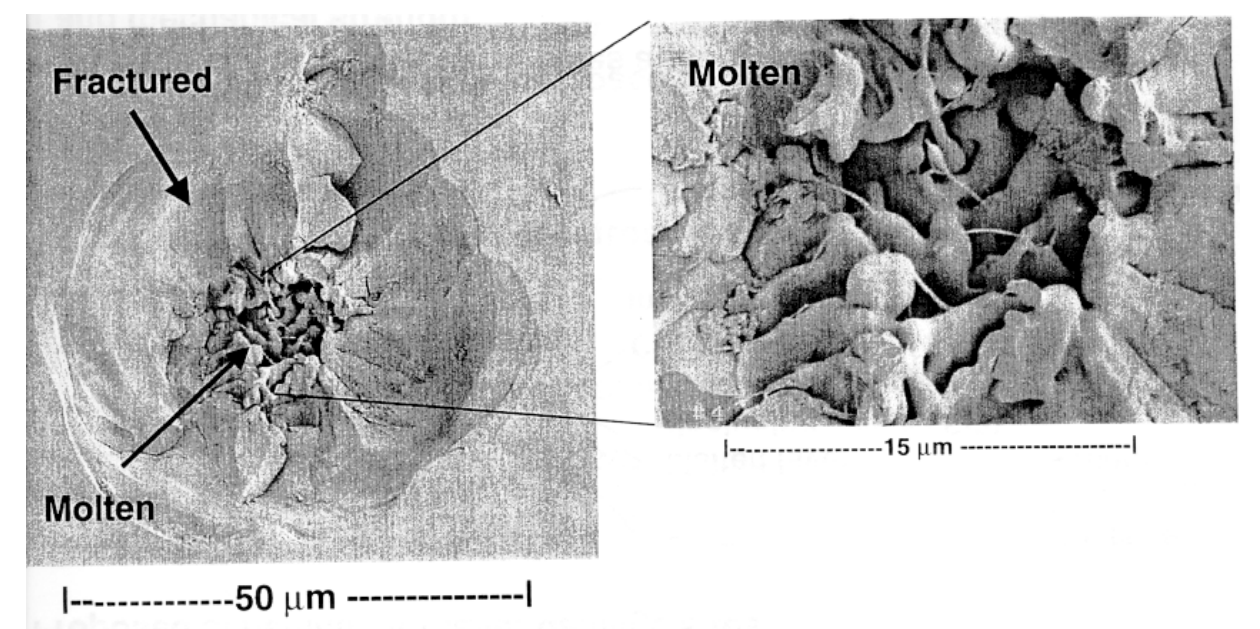

Figure 1.3

From Wong et al.[62, 63]. An STM image of laser-induced damage on the surface of $\mathrm{SiO}_{2}$. The size of the melted material is on the order of the spot size. 


\subsection{The mechanisms by which laser-induced damage occur}

As alluded to above, the phenomenon collectively referred to as laserinduced damage can be separated into the three distinct phases of initiation, energy absorption, and energy dissipation. In the damage initiation phase an initiator (whos nature is discussed below) absorbs sufficient energy to produce an opaque plasma, which then absorbs additional energy rapidly. During energy dissipation, the final phase, the majority of the damage (material modification) occurs from cracking and melting.

Many models have been proposed to account for the initiation mechanisms of laser-induced damage [20, 21, 30, 33, 39, 40, 43-54]. These models can roughly be divided into electronic and material based models. In the electronic models, a sufficient number of electrons are liberated from the valence to the conduction band to produce the opaque plasma, which then absorbs sufficient energy to melt the host material. The original source of the electrons depends on the specific model. Unlike the intrinsic impact ionization model, potential impurities are available to act as an initial source of electrons. If the kinetic energy of a free electron is large enough, a bound electron will be ionized. The free electron population continues to multiply by impact ionization until the plasma is formed. Other potential sources for initial electrons are defect states or electrons liberated by multi-photon ionization.

A distinctly different class of model invokes inclusions of foreign material situated throughout the host matrix. In this type of model the inclusions can act as gray bodies, absorbing a fraction of all light incident on them. The absorbed 
laser radiation would heat the particles until temperatures are hot enough to melt the surrounding host material. The hot material is opaque and absorbs sufficient energy to ionize enough electrons to ignite the plasma. In the following chapters, the nature of each of the three phases of damage will be discussed. In the second and third chapters, it will be demonstrated that the proceeding models are all either incorrect or incomplete. This is accomplished by comparing the wavelength dependence predicted by these proposed models to that experimentally determined in this work. The fourth and fifth chapters will examine the energy absorption and dissipation phases of laser damage. 


\subsection{References}

[1] Maker, P.D., et al., Columbia University Press, New York, 1964: p. 15559.

[2] Hercher, M., J. Opt. Soc. Am. (USA) 54, 536 (1964).

[3] Glezer, E.N., et al., Optics Letters 21, 2023-2025 (1996).

[4] Schaffer, C.B., et al., Opt Express 10, 196-203 (2002).

[5] Efimov, O.M., et al., Opt Mater 17, 379-386 (2001).

[6] Furukawa, Y., et al., Appl Phys Lett 65, 1480-1482 (1994).

[7] Feodorov, V.A., et al., Mater Sci Eng a-Struct Mater 234, 132-134 (1997).

[8] Feit, M.D., et al., SPIE, 1997. 2966: p. 417.

[9] Fedorov, V.A., et al., Tech Phys 43, 1432-1435 (1998).

[10] Demos, S.G., et al., SPIE, 2000. 4347: p. 277.

[11] De Yoreo, J.J., et al., Int Mater Rev 47, 113-152 (2002).

[12] Bhar, G.C., et al., Appl Surf Sci 161, 155-162 (2000).

[13] Azarov, V.V., et al., Kvantovaya Elektron. Mosk. (Russia) 12, 151-4 (1985).

[14] Bass, M., et al., Appl. Opt. (USA) 12, 690-9 (1973).

[15] Barnes, N.P., et al., J. Opt. Soc. Am. (USA) 72, 895-8 (1982).

[16] Campbell, E.M., Fusion Technol 26, 755-766 (1994).

[17] Runkel, M., et al., SPIE, 2001. 4679: p. 408-19.

[18] Sheng, W., et al., SPIE, 2000. 4347: p. 221-7.

[19] Auffarth, G.U., et al., American Journal of Ophthalmology 118, 526-528 (1994).

[20] Bloembergen, N., IEEE J. Quantum Electron. (USA) QE-10, 375-86 (1974).

[21] Braunlich, P., et al., Nucl Instrum Meth Phys Res B 46, 224-230 (1990).

[22] Britten, J.A., et al., Appl. Opt. (USA) 37, 7049-7054 (1998).

[23] Casper, R.T., et al., Nucl Instrum Meth Phys Res B 46, 231-234 (1990).

[24] Dahmani, F., et al., Optics Letters 24, 516-518 (1999).

[25] Dalupang, J.J., et al., Chest 120, 934-940 (2001).

[26] Duthler, C.J., et al., 1976: p. 395-405.

[27] Feit, M.D., et al., SPIE, 1998. 3244: p. 5.

[28] Fradin, D.W., et al., Applied Physics Letters 24, 555-557 (1974).

[29] Gallais, L., et al., Applied Optics 42, 960-971 (2003).

[30] Glass, J., et al., Appl. Opt. (USA) 12, 637-649 (1973).

[31] Gogoll, S., et al., Nuclear Instruments \& Methods in Physics Research Section B-Beam Interactions with Materials \& Atoms 116, 279-283 (1996).

[32] Gupte, S.S., et al., J. Appl. Phys. (USA) 91, 3125-3128 (2002).

[33] Jones, S.C., et al., Optical Engineering 28, 1039-1068 (1989).

[34] Kelly, P., et al., Phys. Rev. B, Condens. Matter (USA) 20, 815-17 (1979).

[35] Lenzner, M., et al., Phys Rev Lett 80, 4076-4079 (1998).

[36] Martin, L.P., et al., Appl Phys Lett 78, 3403-3405 (2001).

[37] Martin, P., et al., Journal of Nuclear Materials 283, 894-897 (2000).

[38] Natoli, J., et al., SPIE, 2002. 4932: p. 224-36.

[39] Schmid, A., et al., SPIE, 1977. 1977: p. 465-72.

[40] Stuart, B.C., et al., Phys Rev Lett 74, 2248-2251 (1995). 
[41] Swain, J., et al., Appl Phys Lett 40, 350-2 (1982).

[42] Taylor, R.S., et al., Appl. Opt. (USA) 27, 3124-34 (1988).

[43] Boyd, R.W., Nonlinear Optics (Academic Press, 2003).

[44] Du, D., et al., Appl Phys Lett 64, 3071-3073 (1994).

[45] Smith, W.L., Opt Eng 17, 489-503 (1978).

[46] Stuart, B.C., et al., Phys. Rev. B, Condens. Matter (USA) 53, 1749-61 (1996).

[47] Singh, A.P., et al., Optics \& Laser Technology 34, 37-43 (2002).

[48] Schmid, A., et al., Phys. Rev. B, Solid State (USA) 16, 4569-82 (1977).

[49] Carr, C.W., et al., SPIE, 2002. 4932: p. 385.

[50] Carr, C.W., et al., SPIE, 2001. 4679: p. 360.

[51] Bloembergen, N., leee J Sel Top Quantum Electr 6, 876-880 (2000).

[52] Bloembergen, N., J Nonlinear Opt Physics Mat 6, 377-385 (1997).

[53] Burns, G., Solid state physics (Academic Press, Orlando, Fla., 1985).

[54] Shen, X.A., et al., Phys Rev Lett 62, 2711-13 (1989).

[55] Rubenchik, A.M., et al., SPIE, 2001. 4679: p. 79.

[56] Koldunov, M.F., et al., Quantum Electronics 32, 623-628 (2002).

[57] Staggs, M., et al., SPIE, 2000. 4347: p. 400.

[58] Yan, M., et al., SPIE, 1996. 2966: p. 11.

[59] Oconnell, R.M., Appl. Opt. (USA) 31, 4143-4153 (1992).

[60] Rainer, F., et al., SPIE, 1992. 1848: p. 46.

[61] Vanstryland, E.W., et al., Phys Rev B 23, 2144-2151 (1981).

[62] Demos, S.G.,Personal Communication "STM image of surface damage" (2003)

[63] Wong, J., et al., SPIE, 2000. 4347: p. 466. 


\section{Chapter 2}

\section{Experimental Apparatus and Technique for Measuring}

\section{the Wavelength Dependence of the Laser-Induced}

\section{Damage Threshold}

\subsection{Introduction}

Unlike lasers, which are driven systems often pumped by flash lamps or other broadband sources, optical parametric oscillators (OPO) are passive devices. When pumped by a laser, an OPO is used for nonlinear conversion of a monochromatic beam into two separate wavelengths. The two resulting beams of light are usually referred to as the signal and idler. As long as energy and momentum are simultaneously conserved, the wavelengths of the output beams are continually tunable[1-4]. The constraints on energy (2.1) and momentum (2.2) can be expressed as:

$$
\hbar \omega_{p}=\hbar \omega_{s}+\hbar \omega_{i}
$$




$$
\frac{n_{p}}{\lambda_{p}}=\frac{n_{s}}{\lambda_{s}}+\frac{n_{i}}{\lambda_{i}}
$$

Although these constraints are conceptually simple, it is not possible to satisfy them in a material with a normal dispersion. This is because the index of refraction in such materials increases monotonically with frequency making it impossible to satisfy condition 2.2. As discussed below, a material which is both birefringent and possesses a non-linear electric susceptibility must be employed to satisfy these conditions. The first constraint is satisfied for any two wavelengths where the sum of the photon energies add up to the energy of the pump photons. A variety of methods may be used to satisfy equation 2.2 , including type I phase matching, type II phase matching, and quasi-phase matching. Type I and II phase matching are similar in that, in both cases, birefringence is used as described below to satisfy equation 2.2 and differ only in the polarizations of the various waves (see table 2.1).

In type I phase matching, as was utilized in this experiment, a uniaxial crystal such as beta barium borate (BBO) can be used as follows. The pump beam is propagated with ordinary polarization (polarization orthogonal to the plane containing the $\mathrm{c}$ axis of the crystal and the propagation vector $\mathrm{k}$ of the pump beam.

\begin{tabular}{|l|l|l|l|}
\hline & Pump & Signal & Idler \\
\hline Type I & Ordinary & Extraordinary & Extraordinary \\
\hline Type II & Ordinary & Ordinary & Extraordinary \\
\hline
\end{tabular}

Table 2.1

Polarizations for type I and type II phase matching in uniaxial crystals (such as the beta barium borate used in these experiments). 
The signal and Idler beam however, propagate with extraordinary polarization. By rotating the crystal around the axis orthogonal to the $\mathrm{c}$ axis and the $\mathrm{k}$ vector, the effective index of refraction for waves traveling with extraordinary polarization is determined by the angle of rotation in equation 2.3. The index of refraction for beams of ordinary polarization is not affected allowing condition 2.2 to be satisfied.

$$
n_{\varepsilon}^{-1}(\theta)=\sqrt{\left(n_{e}^{-1} \sin (\theta)\right)^{2}+\left(n_{o}^{-1} \cos (\theta)\right)^{2}}
$$

Figure 2.1 is a schematic representation of how this is accomplished in an OPO.

Once the phase matching conditions are achieved, it is possible for energy to be transferred from the pump beam to the signal and idler beams. In order to exploit this effect, it is not enough for conversion to be allowed, it must also be efficient. Increasing the length of the conversion medium will only increase efficiency until the pump and converted beam begin to diverge or suffer "walk off". The walk off effect is caused by the pump and converted beams differences in index of refraction. Walk off can be reduced by reversing the orientation of the converting medium periodically, as is done in a ring cavity OPO.

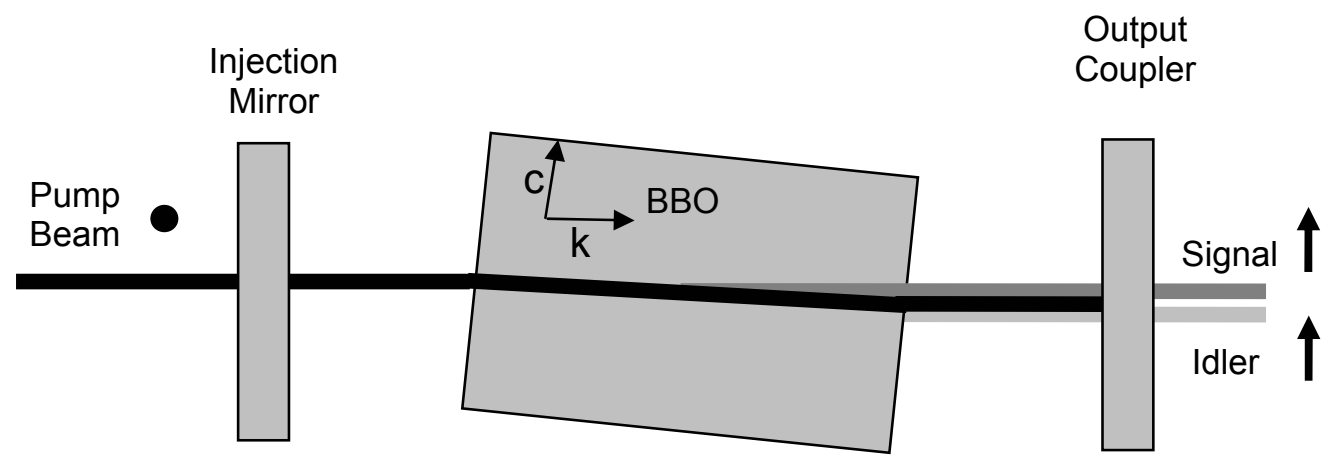

Figure 2.1 
A schematic representation of an OPO The dot next to the pump beam indicates it has ordinary polarization, while the arrows next to signal and idler indicate extraordinary polarization. The injection mirror of an OPO is transparent to the pump beam but highly reflective over a broad range of signal and/or idler wavelengths. The output coupler is only partly reflective over the signal and or idler wavelengths. Placing the converting medium between these two mirrors forms a resonant cavity to stimulate conversion. The converting medium is rotated about an axis orthogonal to the $c$ axis of the crystal and the $\mathrm{k}$ vector of the pump beam to angle tune the effective extraordinary index of refraction.

For these reasons, the conversion must be stimulated if the efficiency is going to be large enough for most applications. This can be accomplished by seeding one of the wavelengths or placing the crystal in a cavity resonant to at least one of the converted wavelength and allowing the wave to grow out of quantum noise. In either case, higher conversion efficiency is achieved by placing the converting medium within a cavity to resonate the signal wave and/or the idler wave as desired. 


\subsection{Experimental methods and techniques}

\section{a. OPO Alignment}

The OPOs used in these experiments were commercially obtained OPOTek "Magic Prism" OPO's of a ring cavity design which use beta-barium borate (BBO) crystals as the nonlinear medium (see figure 2.2). Proper alignment is paramount to efficient operation of the OPO.

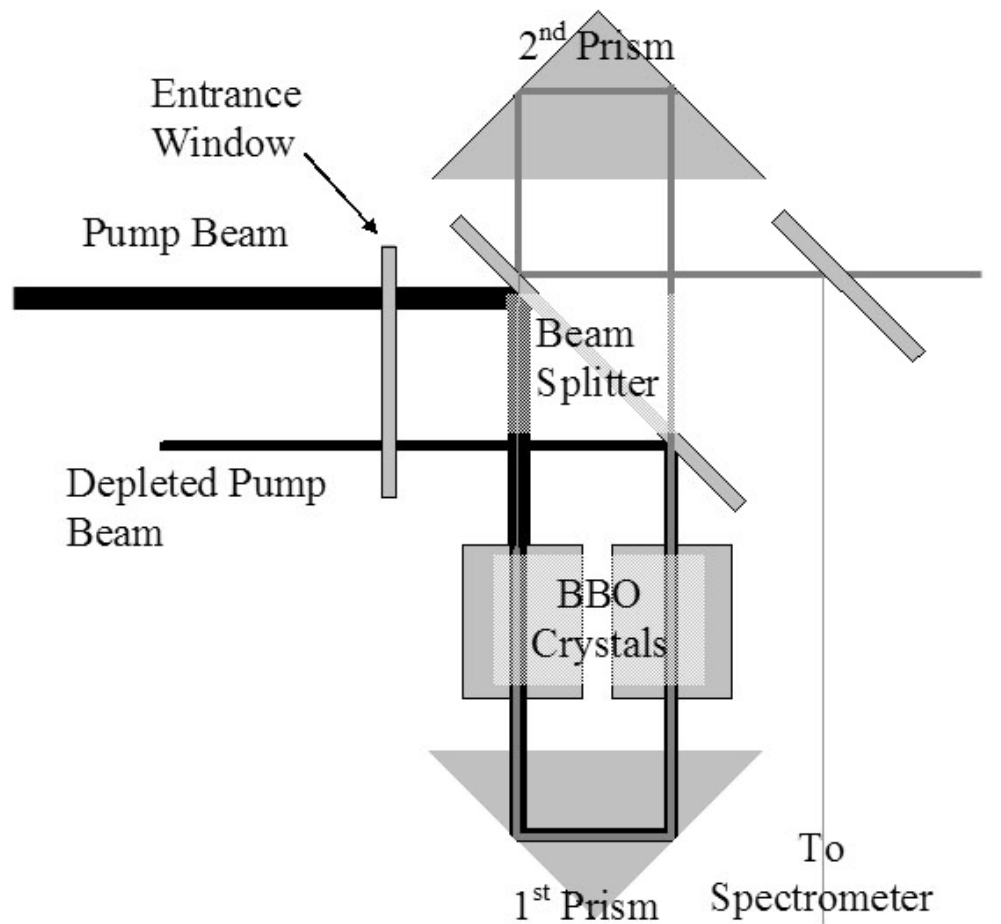

Figure 2.2

A schematic representation of the ring cavity optical parametric oscillator used in these experiments. After passing through the entrance window, the pump beam is turned $90^{\circ}$ by the beam splitter. The pump beam passes through the first BBO where "walk off" occurs during conversion. The second BBO has its optical axis oriented opposite to the first so that the pump and converted beams "walk on" to one another. The unconverted pump beam will be rejected by the beamsplitter after exiting the second BBO. The converted light will pass through the second prism where the majority will be reflected out of the OPO. The remaining light will pass though the beamsplitter to stimulate conversion in the BBO during the remainder of the pump pulse. Because the optical path of the cavity formed by the two prisms is only 4 inches in length, portions of the light converted in the first part of the 3 ns pulse will make up to 9 passes in the cavity before the termination of the pump pulse. 
In order to achieve the desired alignment, the pump beam must be precisely aligned to the optical axis when traveling through the BBO crystals. This is best achieved, both for reasons of safety and efficiency, by using a low power HeNe laser aligned to co-propagate with the pump beam. 


\section{b. Experimental set up.}

The laser-induced damage threshold in DKDP has been observed to vary by up to two orders of magnitude when focal geometry, pulse duration, polarization, and wavelength of the damaging beam are not stringently controlled. For this reason an OPO is the only practical source of radiation to probe the wavelength dependence of the damage threshold. Unfortunately, the properties which make the OPO indispensable to the experiment also make it extremely difficult to use. The experimental set up used in these experiments can be seen in figure 2.3 .

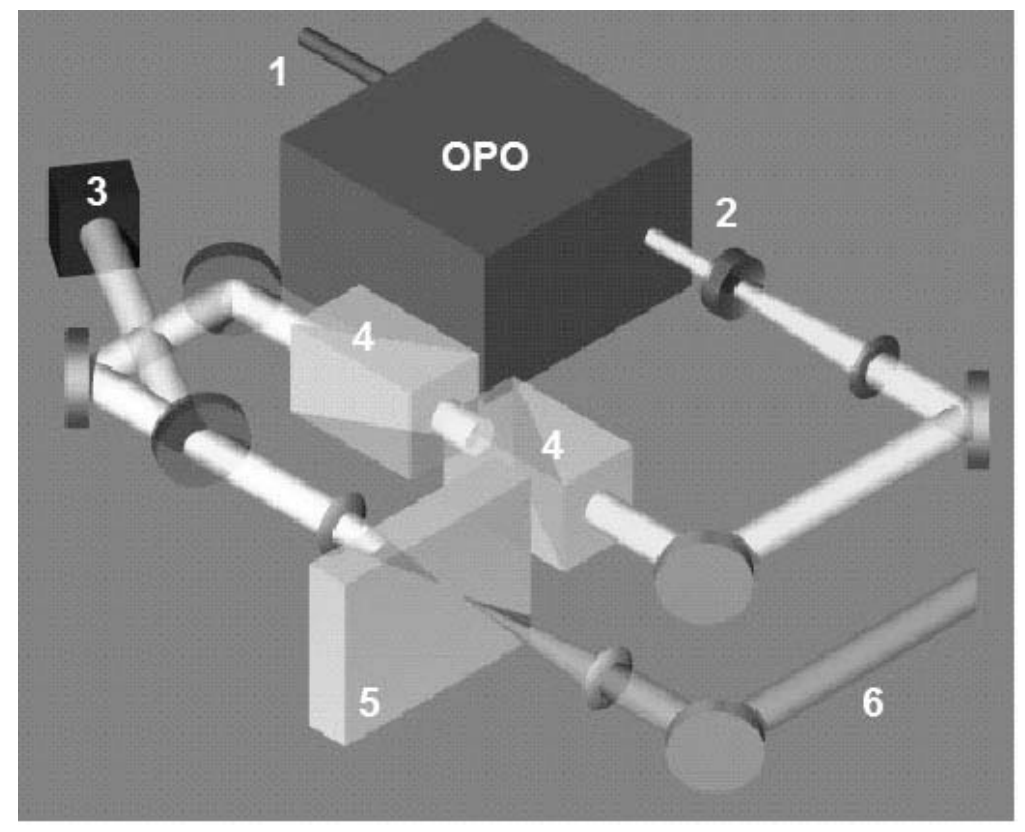

Figure 2.3

1) The OPO is pumped at $266 \mathrm{~nm}$ or $355 \mathrm{~nm}$ to produce a continuous output from $310 \mathrm{~nm}$ to $650 \mathrm{~nm}$. 2) A Keplerian telescope is used to correct the wavelength dependent divergence. 3) The energy of each pulse is measured by a computer monitored thermopile energy meter. 4) Pulse energy is regulated by a pair of computer controlled polarizers which also establish a polarization purity of $1000 \mathrm{~S}: 1 \mathrm{P}$. The beam is focused by a compound lens system into the bulk of a 5) DKDP sample. 6) A cw HeNe laser is focused into the same region through the back surface of the sample. 
In order to effectively use the diverging output from the OPO, a telescope was used to expand and collimate the beam. Because the output beam of the OPO suffers from a wavelength dependent divergence, this telescope was necessary to re-collimate the beam whenever the wavelength of the OPO was altered. Broadband High Reflectivity (HR) mirrors were used to transport the OPO output to reduce the losses that would be expected from metal mirrors. In addition to the wavelength dependence, the divergence of the OPO is severely sensitive to pump energy. It was therefore necessary to pump the OPO at a constant fluence, regardless of the desired energy at the sample, and regulate the pulse energy with a pair of broadband polarizers. This arrangement had the additional benefit of producing a beam with a polarization extinction ratio of 1000 $\mathrm{S}: 1 \mathrm{P}$. This was possible because the first polarizer was mounted on a computer controlled rotation stage, while the second was held at a fixed orientation. 


\section{c. Diagnostics}

Pulse energy and duration were monitored by use of a wedge which reflected a pair of low energy beams into an energy meter and a photodiode (see figure 2.4).

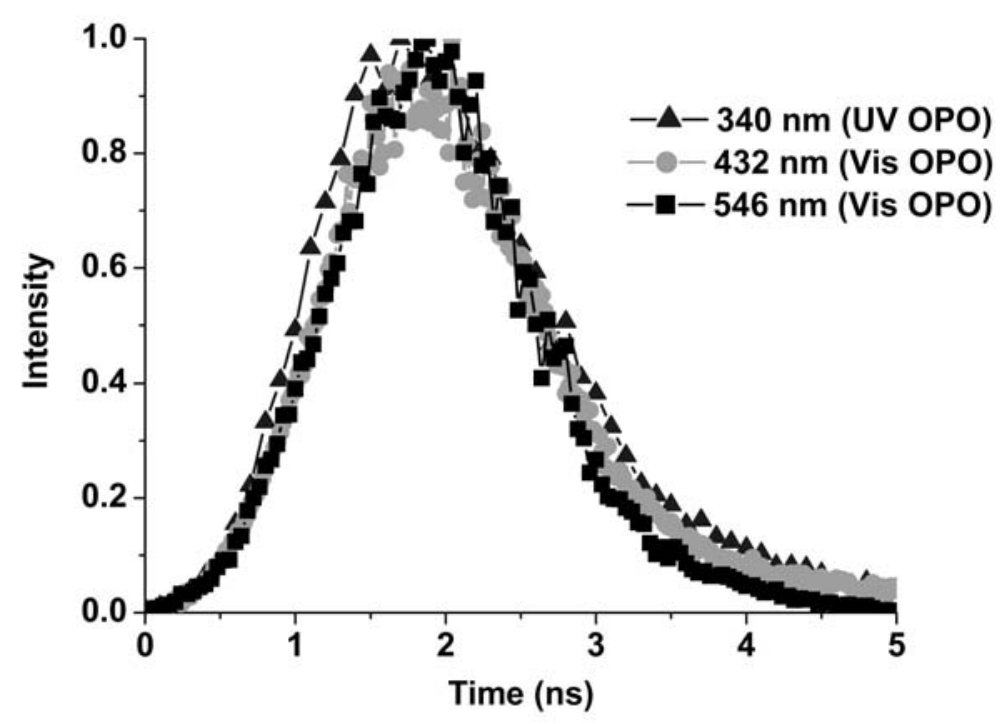

Figure 2.4

The pulse duration produced by the OPO at $340 \mathrm{~nm}, 432 \mathrm{~nm}$, and $546 \mathrm{~nm}$ as measured with a photodiode and oscilloscope.

Once the beam has passed through the diagnostic and control optics, it is then focused into the bulk of the sample with a spot size held constant by the compound lens system described below.

A laser beam of diameter $d$ and wavelength $\lambda$ focused by a simple lens or mirror of focal length $f$ will ideally produce a spot size of $w_{0}$ as given by equation 2.4 .

$$
w_{0}=\frac{4 \pi \cdot f \cdot \lambda}{D}
$$


In order to maintain a constant spot size over wavelength range of several hundred $\mathrm{nm}$ it was necessary to use a compound lens system which simulated a lens with a continuously adjustable focal length.

The focusing system consisted of two lenses of nominal focal lengths (the focal length of the lenses themselves is governed in part by the wavelength of light used) of $+100 \mathrm{~mm}$ and $-100 \mathrm{~mm}$. The separation of the two lenses was adjusted to obtain a spot size of $100 \mu \mathrm{m}+/-5 \mu \mathrm{m}$ at every wavelength and the distance between the lens system and sample was tuned to keep the focal spot of the beam in the center of the sample.

$$
\frac{1}{f_{\varepsilon}}=\frac{1}{f_{1}}+\frac{1}{f_{2}}-d \frac{f_{1} \cdot f_{2}}{f_{1}+f_{2}}
$$

The focal geometry was monitored by imaging a very low energy beam with a CCD put in place of the sample. Images of two focal spots, separated by approximately $200 \mathrm{~nm}$ can be seen in figure 2.5 .

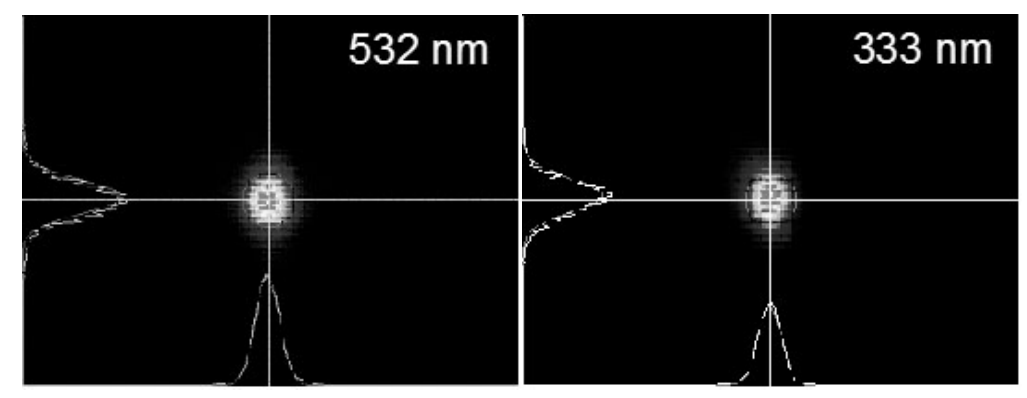

Figure 2.5

The focal spot geometry showing the beam waist for wavelengths of $532 \mathrm{~nm}$ and $333 \mathrm{~nm}$.

By perturbing the chip of the CCD about the desired focal plane the size, geometry, Rayleigh range, and location of the beam waist could be evaluated precisely (see figure 2.6). 


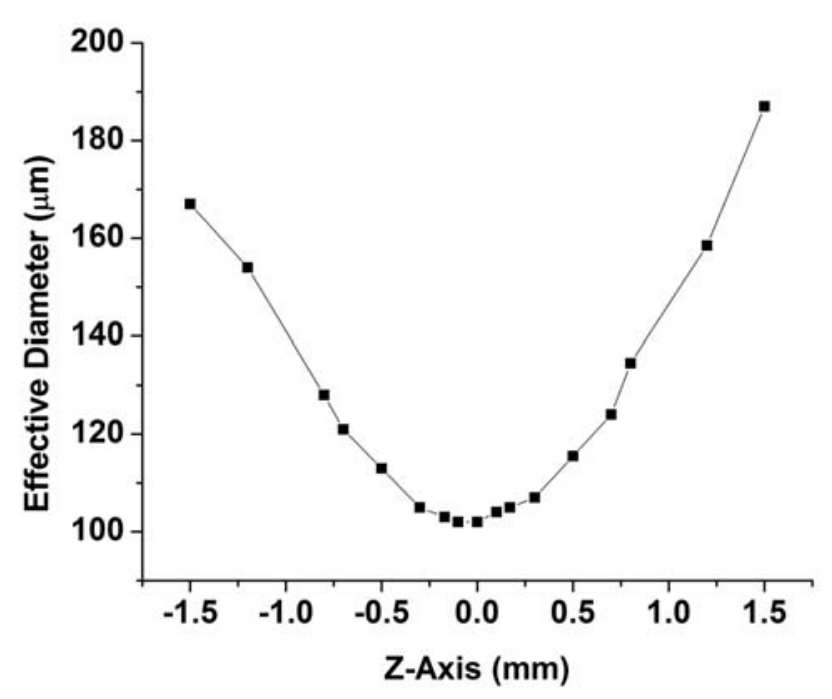

Figure 2.6

By imaging the spot size at several places along the $z$ axis and plotting the effective diameter versus the position, the beam waist can be positively identified. The Rayleigh range is seen to be on the order of $500 \mu \mathrm{m}$.

Measuring the spot diameter was standard diagnostics and was performed for all the wavelengths used in this experiment. The beam waist as a function of wavelength is shown in figure 2.7 . 


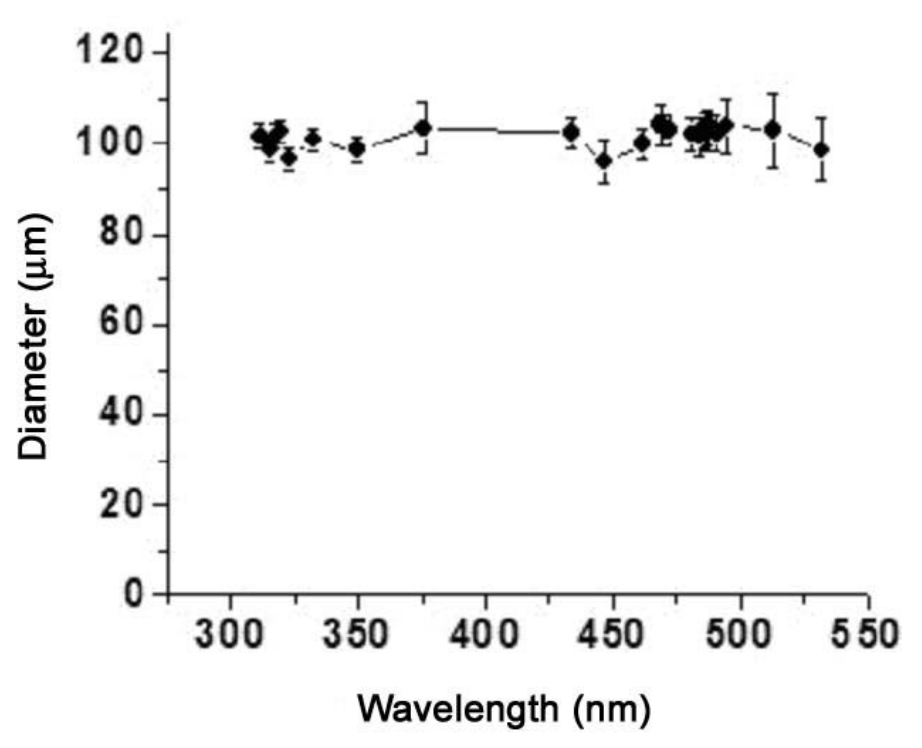

Figure 2.7

The beam waist was maintained at $100 \mu \mathrm{m}+/-5 \mu \mathrm{m}$ for all the wavelengths used in this experiment.

The final diagnostic was a verification of the wavelength at which a measurement was performed. This was accomplished by running a fiber from the low energy output of the OPO to a spectrometer. In addition to verifying the wavelength used in experiments, the line width of the pulse was determined to be on the order of $1 \mathrm{~nm}$ (see figure 2.8). The wavelength was controlled by adjusting a manual micrometer on the OPO. In practice it proved more practical to calibrate the arbitrary units on the micrometer to wavelength. The calibration look up table produced accurate and repeatable wavelength tuning up to the resolution of the spectrometer. 


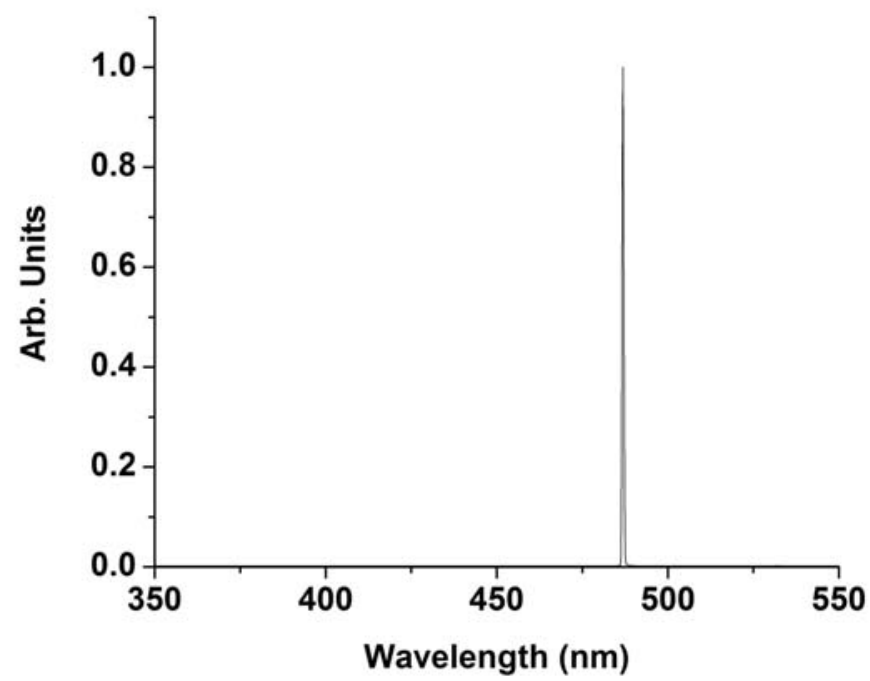

Figure 2.8

The output of the OPO at $487.0 \mathrm{~nm}$ depicts a typical line width of $1 \mathrm{~nm}$. 


\subsection{Data collection}

Once the diagnostics were complete, the data were collected by irradiating virgin (not previously exposed to high energy laser radiation) sites in the bulk of a single DKDP crystal with individual pulses. An image of each site was taken before and after the pulse by a CCD imaging system with $15 \mu \mathrm{m}$ spatial resolution. (see figure 2.9)

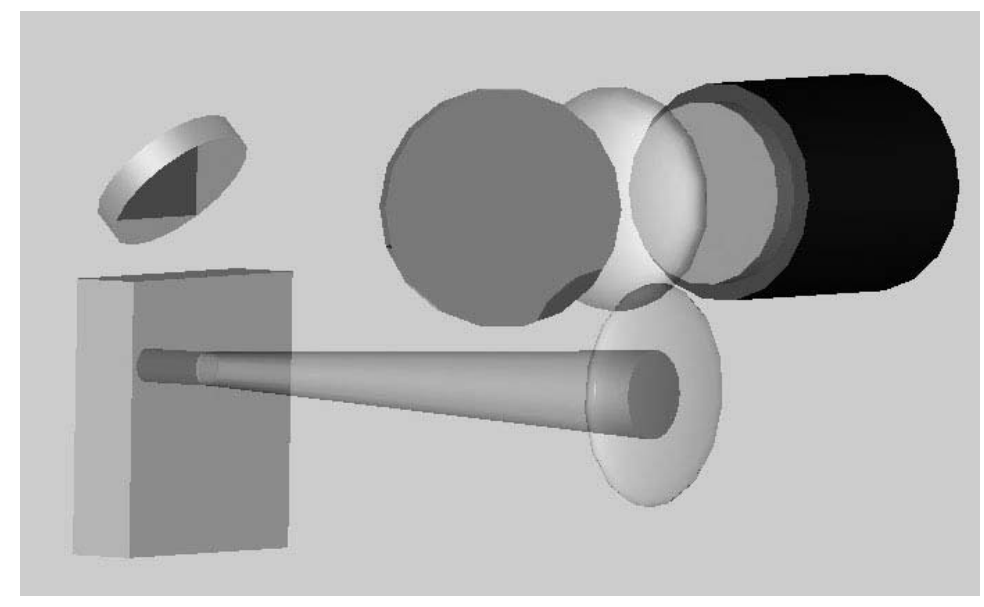

Figure 2.9

The CCD imaging system used for damage detection consisted of an 8 bit camera $632 \mathrm{~nm}$ band pass filter, and a focusing lens. The system was mounted on a computer controlled electromechanically actuated stage so that the focus could be adjusted by the damage acquisition software.

The after image was subtracted from the before image resulting in a difference image which was examined by eye to ascertain if damage had occurred. The fluence of the laser pulse and the binary determination of damage were incorporated in real time into a damage probability histogram. The histogram was a plot of the fraction of times a specified fluence range produced damage. Data were collected at a given wavelength until the histogram 
converged into a stable configuration, which was always observed to be a monotonic "s-curve". The extensive bookkeeping required for the experiment was minimized by use of a labview program which automated every aspect of the data acquisition except for making a judgment as to whether damage occurred (see figure 2.10)

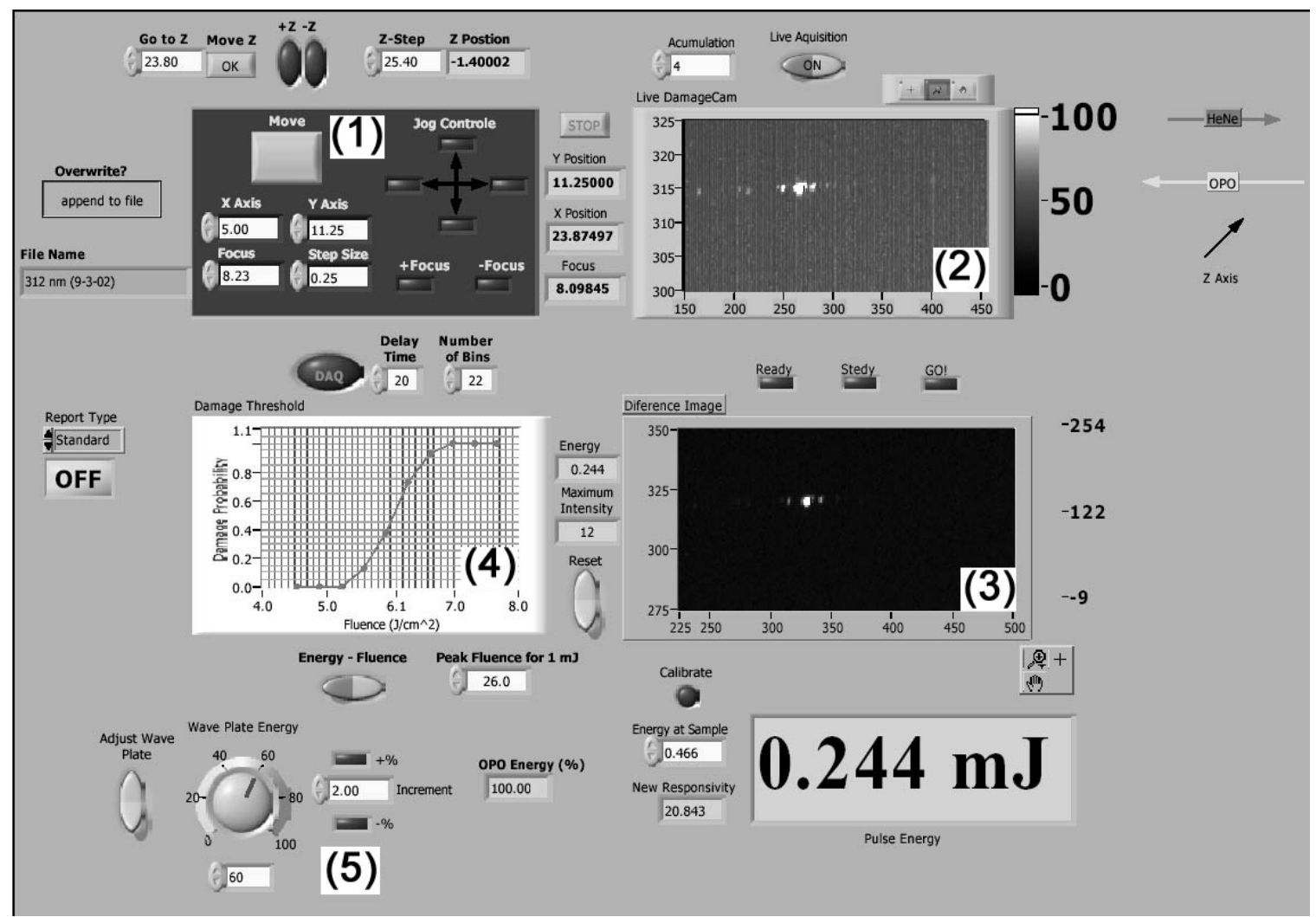

Figure 2.10

The front panel of the data acquisition program. 1) The program controlled sample positioning and camera focus by commanding electromechanically actuated micrometers. 2) The damage detection camera had a spatial resolution of $15 \mathrm{um}$ and could display live video or averaged images. 3) Before and after images from the damage detection camera were automatically subtracted to enhance damage detection sensitivity. 4) The energy of the laser pulse was automatically retrieved from the thermopile energy meter and combined with the beam profile data to determine the peak fluence of the shot. This information and the user determination of damage were entered into the damage probability vs. fluence histogram in real time. 5) Pulse energy was adjusted by a pair of polarizers. The polarizer was mounted in an electromechanically actuated rotation stage. The second polarizer was in a fixed orientation and set the polarization of the incident laser light to $1000 \mathrm{~S}: 1 \mathrm{P}$. 


\section{$2.4 \quad$ References}

[1] Harris, S.E., Proceding IEEE 57, 2096 (1969).

[2] Koechner, W., Solid State Laser Engineering (Springer-Verlag.

[3] Boyd, R.W., Nonlinear Optics (Academic Press, 2003).

[4] Siegman, A., Lasers (University Science Books, 1986). 


\section{Chapter 3}

\section{Analysis of Wavelength Dependent Damage Threshold}

\section{Data}

\subsection{Introduction}

In this chapter the data collected by the methods described in Chapter 2 are analyzed. The global response of the damage initiation fluence as a function of wavelength is examined by compiling the fluences at which a DKDP crystal is damaged $50 \%$ of the time for a range of wavelengths. Distinct steps whose width is of order $\mathrm{K}_{\mathrm{b}} \mathrm{T}$ are observed in the damage threshold at photon energies associated with the number of photons $(3 \rightarrow 2$ or $4 \rightarrow 3)$ needed to promote a ground state electron across the energy gap. The wavelength dependence of the damage threshold suggests that a primary mechanism for damage initiation 
in DKDP is a multi-photon process in which the order is reduced through excited defect state absorption. 


\subsection{Data}

S-curves are generated by testing on the order of 100 sites at each wavelength; the procedure for which is described in detail in chapter 2. Figures 3.1 to 3.3 show the s-curves for each of the 21 wavelengths for which the damage threshold was measured. The experiments were conducted explicitly to determine the fluence at which the crystal would damage $50 \%$ of the time (the $50 \%$ damage threshold fluence or $50 \% \mathrm{DTF}$ ); and hence the majority of data points were taken near $50 \%$ DTF for each wavelength. This is reflected in the error associated with the data in figures 3.1 to 3.3 . Typical errors at $50 \%$ DTF level are $5 \%$ or less. The values at the extremes (10\%DTF and $90 \%$ DTF) can have errors as large as $20 \%$. The error bars are omitted from the plots for clarity. Experiments performed at wavelengths between $300 \mathrm{~nm}$ and $400 \mathrm{~nm}$ used the 266-nm pumped OPO. The remainder of the experiments were performed at wavelengths between $434 \mathrm{~nm}$ and $532 \mathrm{~nm}$, using the 355-nm pumped OPO as the illumination source. 


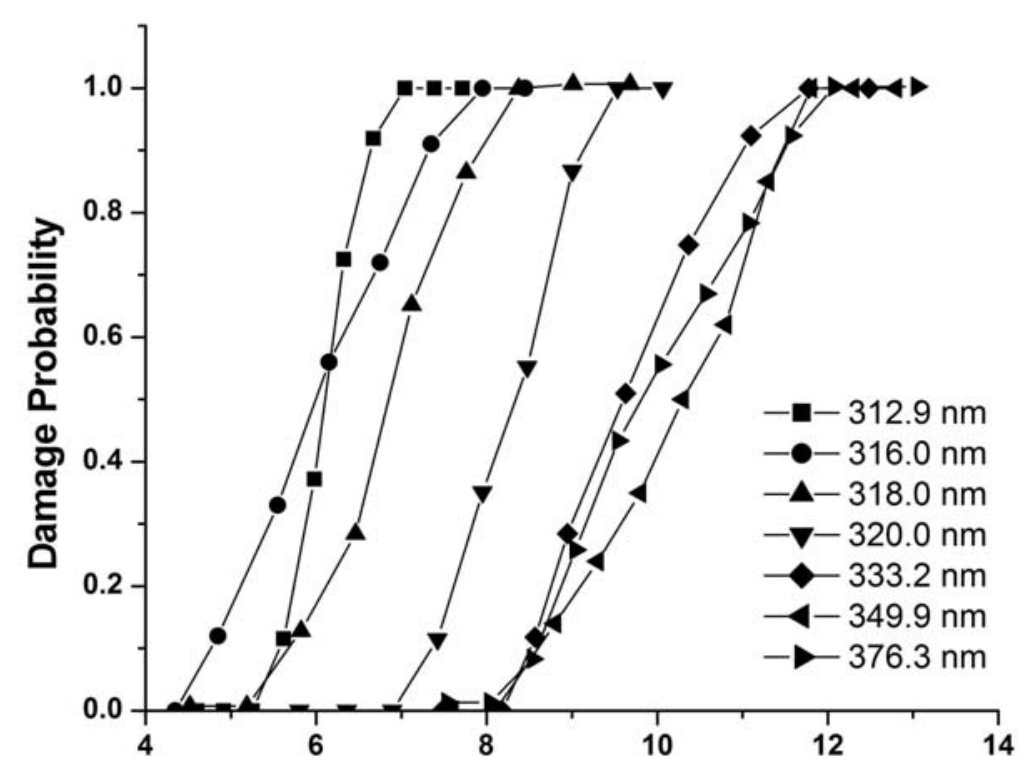

Fluence $\left(\mathrm{J} / \mathrm{cm}^{2}\right)$

Figure 3.1

S-curves for $312.9 \mathrm{~nm}(3.96 \mathrm{eV})$ to $376.3 \mathrm{~nm}(3.29 \mathrm{eV})$ The 266-nm pumped OPO was the light source used in obtaining this data.

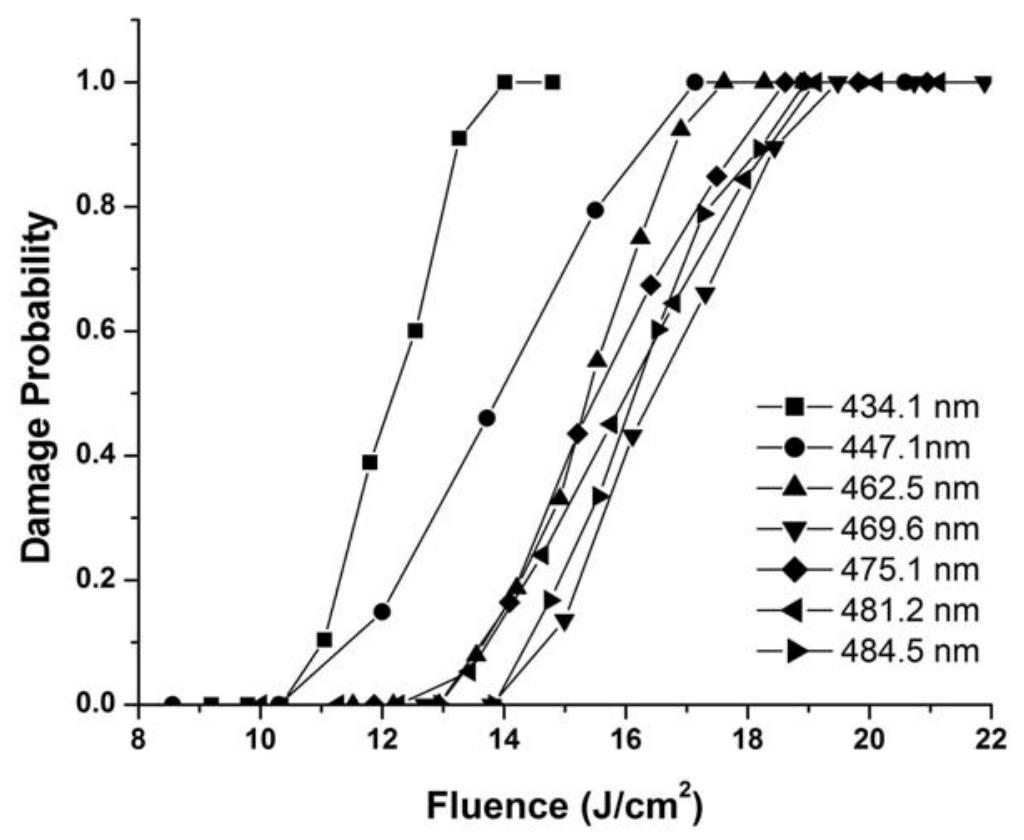

Figure 3.2

S-curves for $434.1 \mathrm{~nm}(2.86 \mathrm{eV})$ to $484.5 \mathrm{~nm}(2.56 \mathrm{eV})$. The $355-\mathrm{nm}$ pumped OPO was the light source used in obtaining this data. 


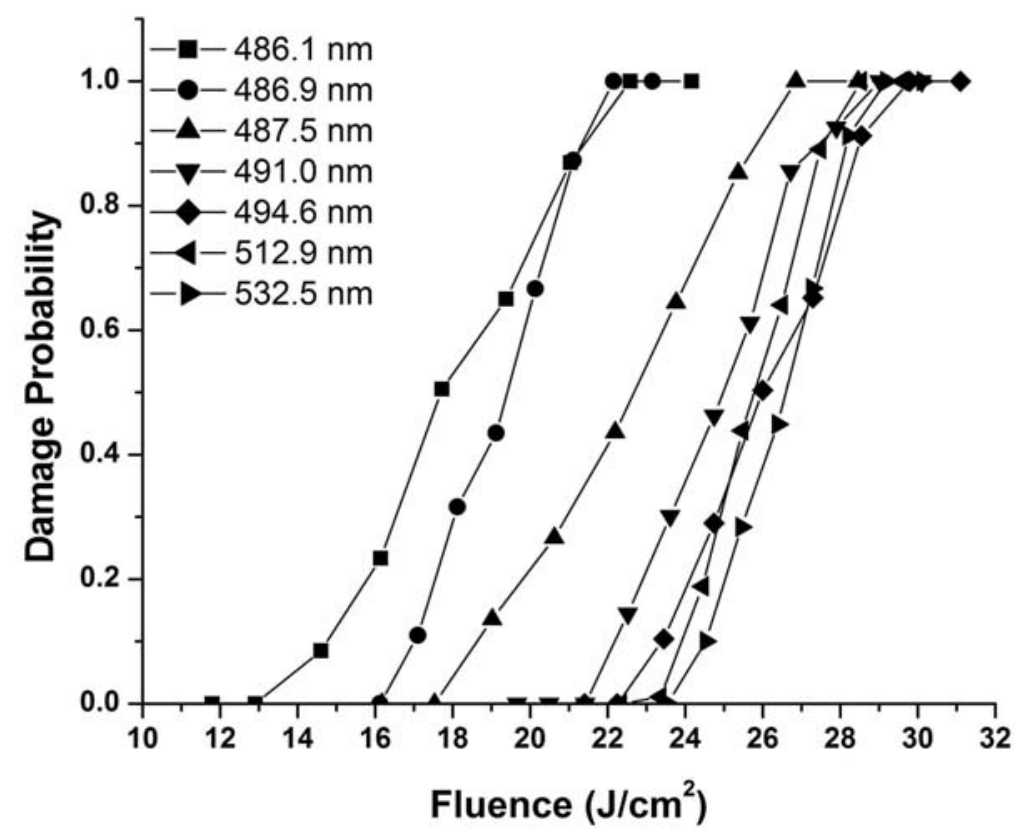

Figure 3.3

S-curves for $486.1 \mathrm{~nm}(2.55 \mathrm{eV})$ to $532.5 \mathrm{~nm}(2.32 \mathrm{eV})$. The $355-\mathrm{nm}$ pumped OPO was the light source used in obtaining this data. 


\subsection{Data analysis}

In figures 3.4 to 3.6 the fluence at which each of the 21 s-curves cross $50 \%$ damage probability are plotted. This is accomplished by rescaling the damage probability axis in figures 3.1 to 3.3 in order to show only the fluence at which the sample damage $50 \%$ of the time at each wavelength: the $50 \%$ DTF.

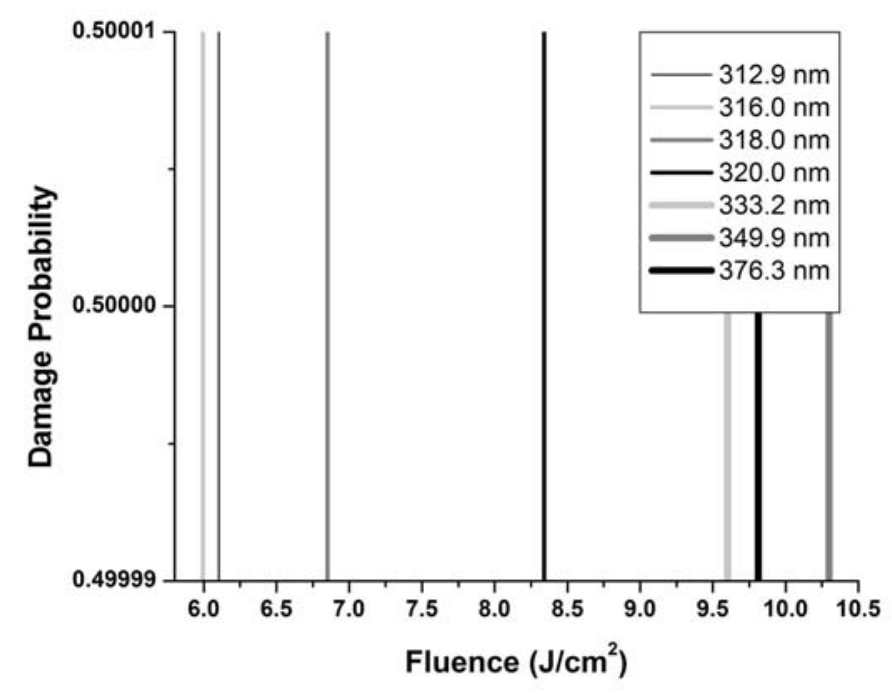

Figure 3.4

The fluences at which each of the 7 wavelengths between $312.9 \mathrm{~nm}$ and 376.3 $\mathrm{nm}$ produced damage $50 \%$ of the time is seen by rescaling the vertical axis of figure 3.3. 


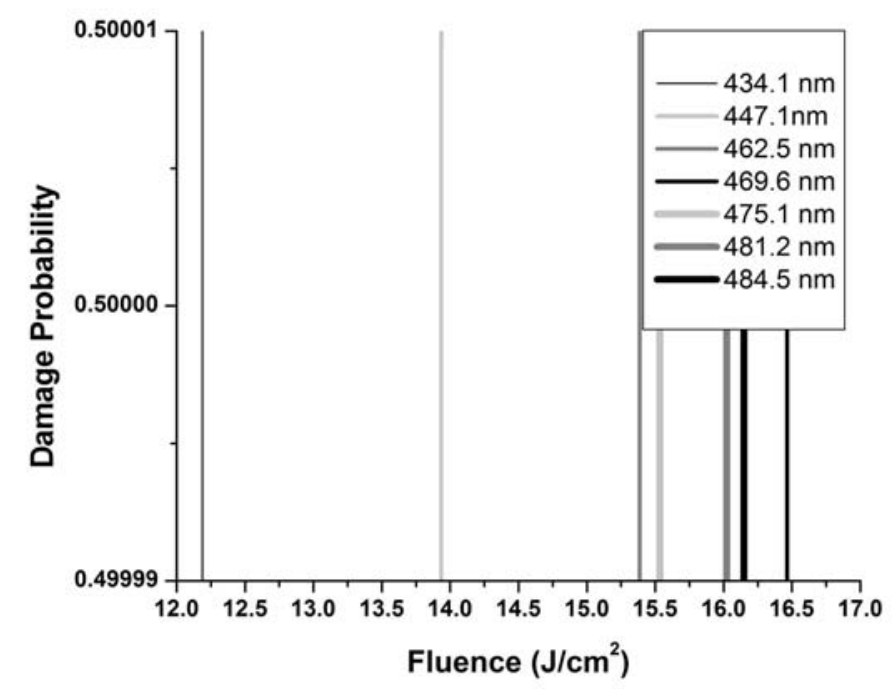

Figure 3.5

The fluences at which each of the 7 wavelengths between $434.1 \mathrm{~nm}$ and 484.5 $\mathrm{nm}$ produced damage $50 \%$ of the time is seen by rescaling the vertical axis of figure 3.4.

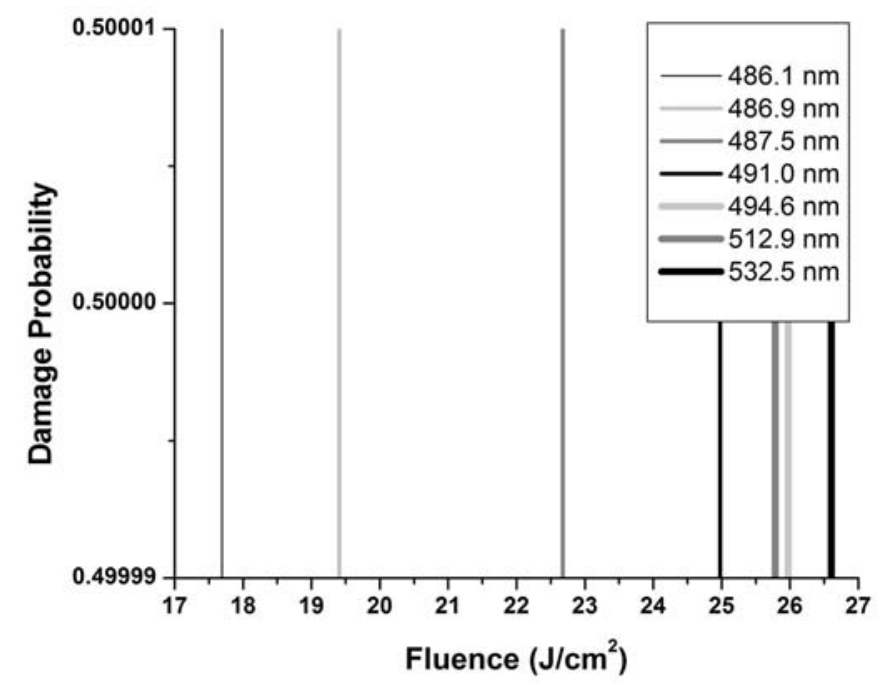

Figure 3.6

The fluences at which each of the 7 wavelengths between $486.1 \mathrm{~nm}$ and 532.5 $\mathrm{nm}$ produced damage $50 \%$ of the time is seen by rescaling the vertical axis of figure 3.5.

Ordered pairs are created from the wavelength of an s-curve, and the $50 \%$ DTF (the values indicated in figures 3.4 to 3.6 ). It must be emphasized that 
this is the fluence at which a $100 \mu \mathrm{m}$ diameter spot will damage the crystal $50 \%$ of the time at that particular wavelength. As discussed in Chapter 1, the damage threshold depends on the spot size. Plotting these ordered pairs gives a global representation of the relation of wavelength to damage threshold (see figure 3.7).

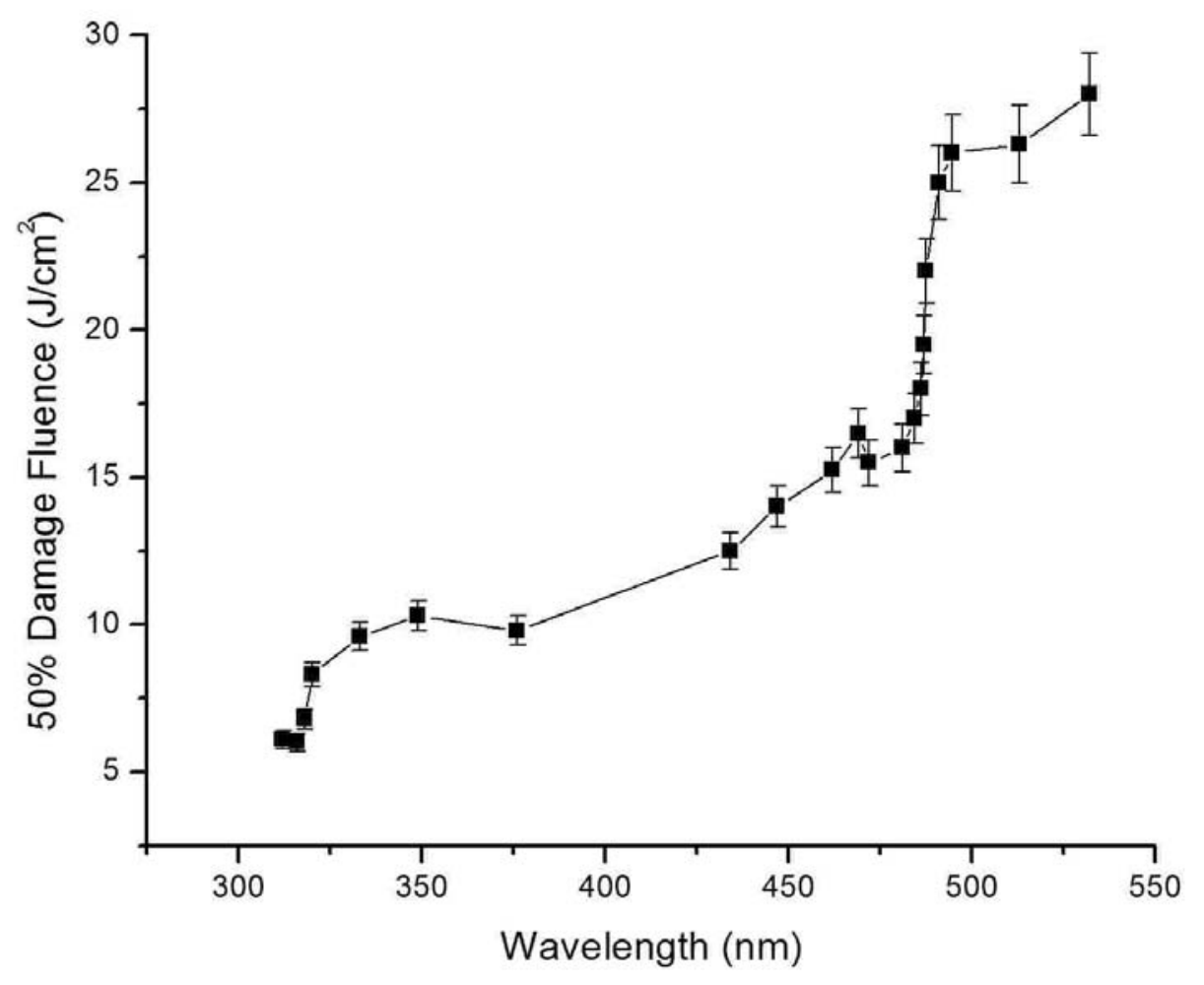

Figure 3.7

The wavelength dependent damage threshold is obtained by plotting wavelength vs. the fluence at which the sample damaged $50 \%$ of the time at a particuler wavelength.

Figure 3.7 displays a number of striking features; most notable are the sharp steps in the damage threshold centered at $487 \mathrm{~nm}(2.55 \mathrm{eV})$ and $318 \mathrm{~nm}$ $(3.90 \mathrm{eV})$. The region in between the steps exhibits a smooth decrease in the damage threshold with decreasing wavelength. 
Replotting the s-curve data in terms of photon energy at a constant fluence vs.

damage probability across each transition provides additional information regarding the nature of the transitions (see figure 3.8 and 3.9).

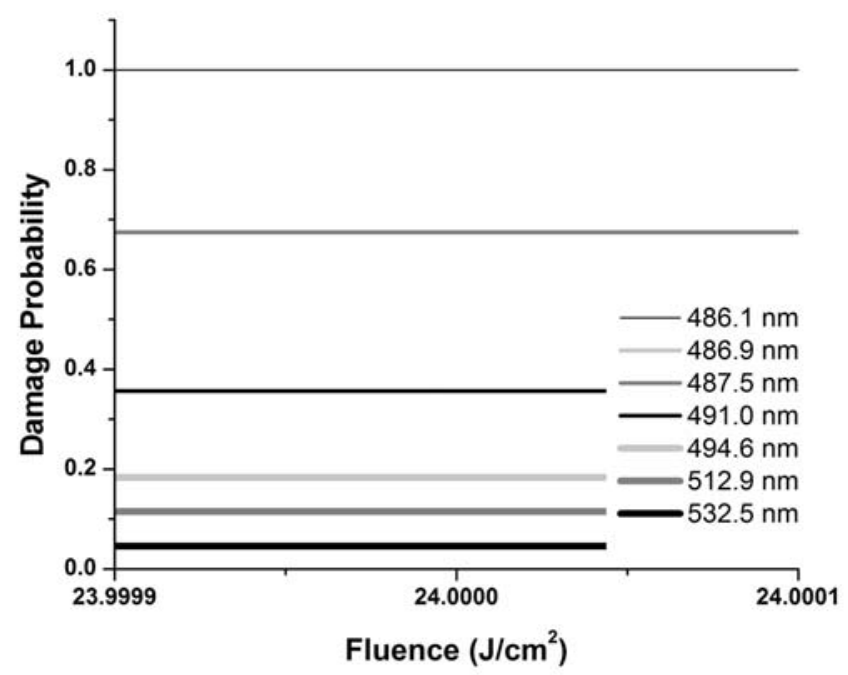

Figure 3.8

Damage probability for $24 \mathrm{~J} / \mathrm{cm}^{2}$ for wavelengths near the $487 \mathrm{~nm}$ step. The damage probabilities for $486.1 \mathrm{~nm}$ and $486.9 \mathrm{~nm}$ are both unity.

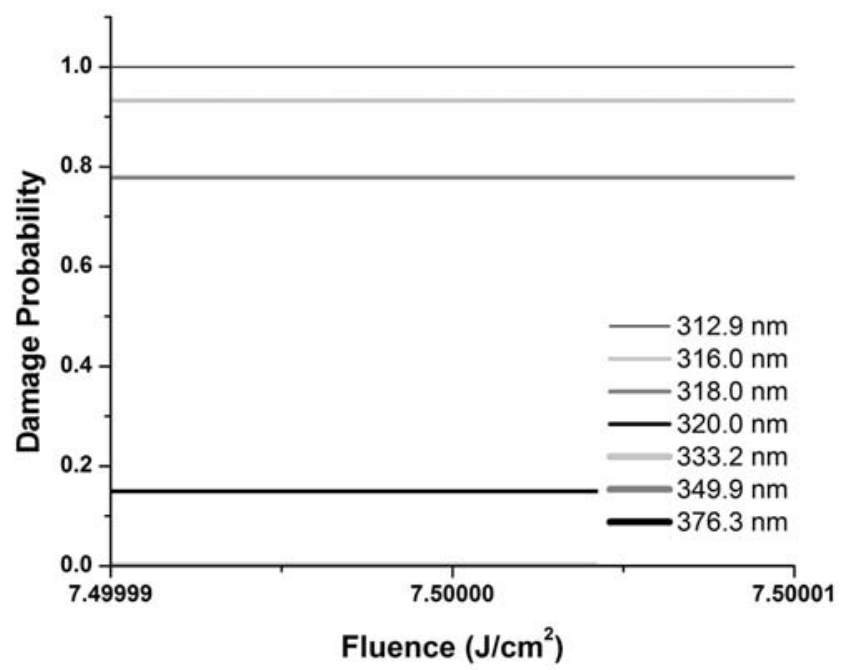

Figure 3.9

Damage probability for $7.5 \mathrm{~J} / \mathrm{cm}^{2}$ for wavelengths near the $319 \mathrm{~nm}$ step. The damage probabilities for $333.2 \mathrm{~nm}, 349.9 \mathrm{~nm}$, and $376.3 \mathrm{~nm}$ are al zero. 
This is done by selecting a single fluence and plotting the probability of damage as a function of photon energy. For example, figure 3.8 shows that, at a wavelength of $491.0 \mathrm{~nm}$ and a fluence of $24 \mathrm{~J} / \mathrm{cm}^{2}$ damage is expected $36 \%$ of the time, while a $487.5-\mathrm{nm}$ pulse of the same fluence has a $66 \%$ probability of producing damage.

In figure 3.10 , the probability of damage at a fixed fluence is plotted vs. photon energy for the transitions at $2.55 \mathrm{eV}$ and $3.90 \mathrm{eV}$. The fluence at the top of the each step was used, $24 \mathrm{~J} / \mathrm{cm}^{2}$ and $7.5 \mathrm{~J} / \mathrm{cm}^{2}$, respectively.

When the midpoints of the transitions are overlapped (by subtracting $2.55 \mathrm{eV}$ and $3.9 \mathrm{eV}$ from the data surrounding the first and second steps respectively) it becomes evident that both steps exhibit the same characteristics (see figure 3.10). The width of the transitions is established in figure 3.10 to be on the order of $\mathrm{K}_{\mathrm{b}} \mathrm{T}$ at room temperature.

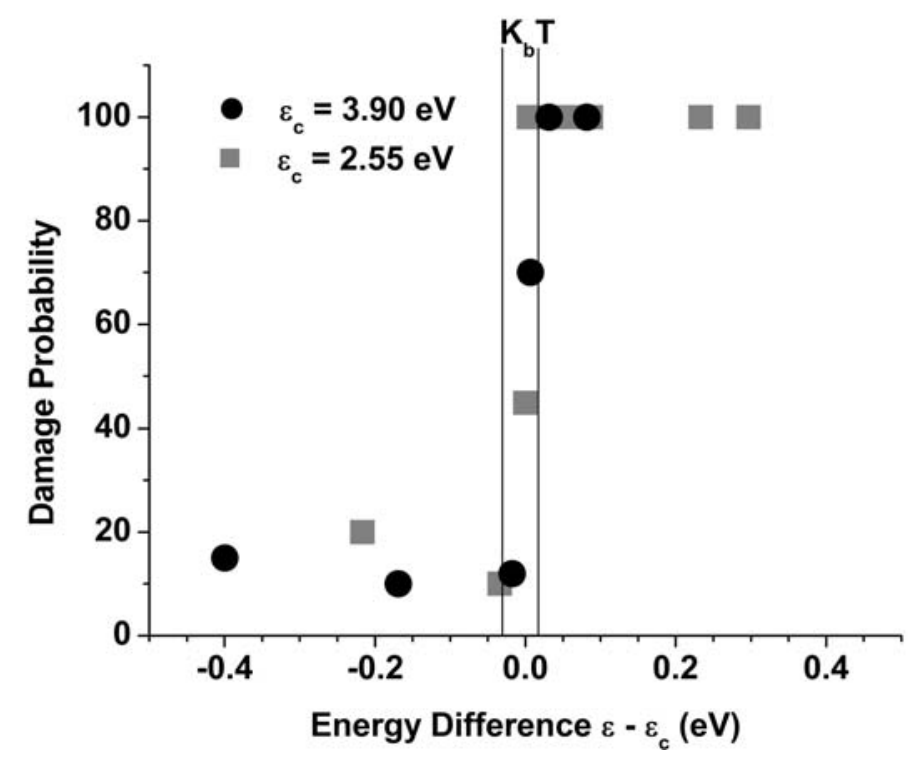

Figure 3.10

Damage probability at fixed fluences plotted vs. photon energy difference from each damage step $\left(\varepsilon_{\text {photon }}-\varepsilon_{c}\right)$. The width of the transition is approximately $\mathrm{K}_{\mathrm{b}} \mathrm{T}$. 


\subsection{Discussion}

To compare the energy at which the steps in the damage threshold occur with respect to the DKDP band gap $\left(\varepsilon_{g}\right)$, it was first necessary to determine the nature of $\varepsilon_{g}$ by UV transmission measurements. From the extinction of transmission, a lower bound to the band gap is determined to be $\varepsilon_{g \mathrm{~L}}=7.5 \mathrm{eV}$. The band gap is generally identified by an absorption coefficient of $10^{4} \mathrm{~cm}^{-1}$ [1]. The absorption coefficient of our sample was only $100 \mathrm{~cm}^{-1}$ at $7.5 \mathrm{eV}$, while the band gap is potentially as high as $9 \mathrm{eV}$ [2] (see figure 3.11).

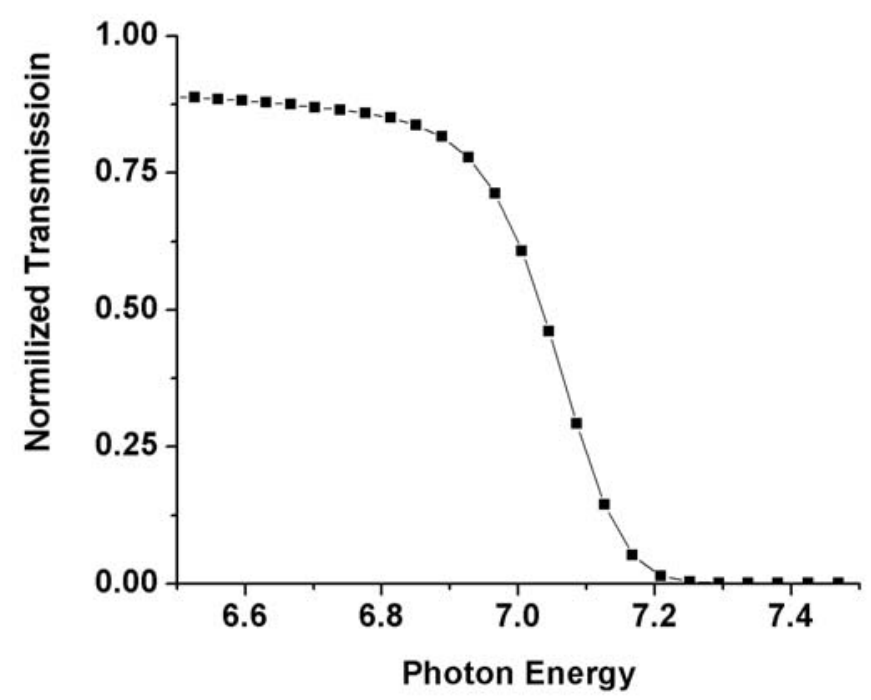

Figure 3.11

Normalized transmission through a 1-cm thick piece of DKDP.

The limiting factor in the UV absorption measurement was the thickness of the sample. Because of the brittle nature of the material it proved impossible to produce DKDP samples of high surface quality thinner than $0.9 \mathrm{~mm}$. Low temperature photoluminescence measurements of KDP show a band gap 
between 8.0 and $8.8 \mathrm{eV}[3]$. Recent theoretical calculations suggest that the band gap of the two closely related materials should be similar if not identical [4].

The experimental results demonstrate the presence of sharp reductions in the damage probability at photon energies in the vicinity of fractions of an effective band gap $\varepsilon_{g} / n, n=2,3$. In addition, the width of both steps is very narrow $\left(\approx K_{b} T\right)$ while the damage morphology indicates the presence of discrete initiation sites (see figure 3.12).

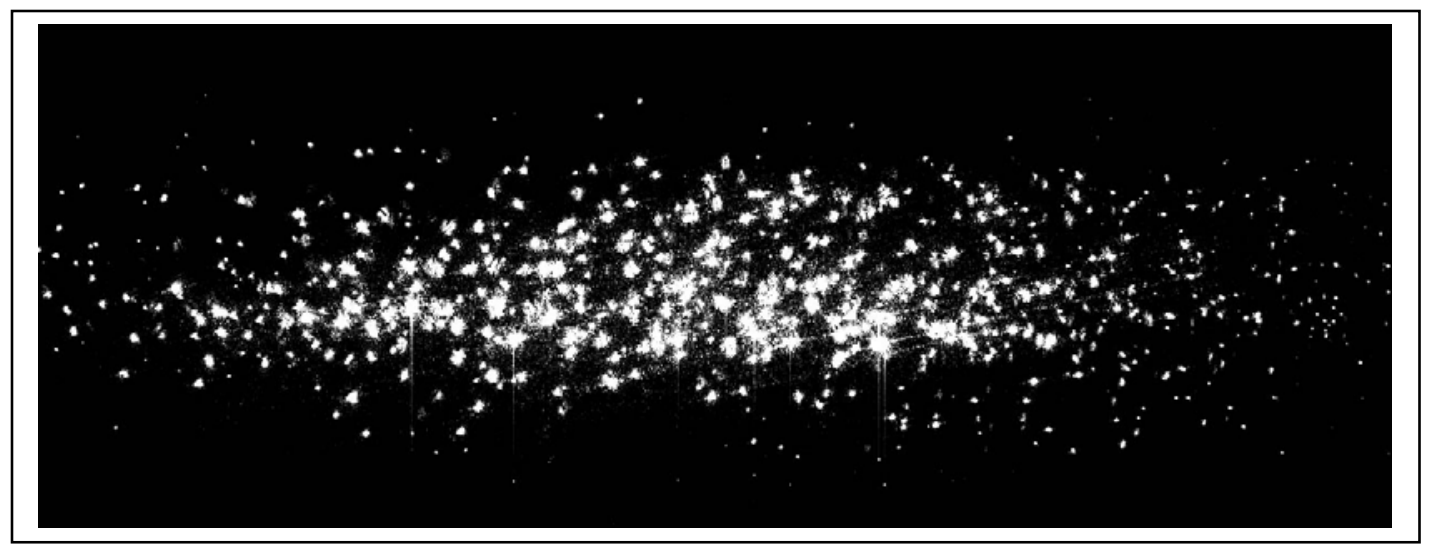

Figure 3.12

Light scattering image of DKDP material modified by a 355-nm 3-ns laser pulse. The laser pulse propagated from left to right. The fluence distribution of the beam was Gaussian in the vertical direction. The image shows that the damage is not restricted to the peak fluence, but distributed through the beam.

The decrease in damage threshold across the steps is approximately from 27 $\mathrm{J} / \mathrm{cm}^{2}$ to $16 \mathrm{~J} / \mathrm{cm}^{2}$ and from $9 \mathrm{~J} / \mathrm{cm}^{2}$ to $6 \mathrm{~J} / \mathrm{cm}^{2}$, respectively.

By considering these experimental features, an explanation for the damage mechanism can be proposed. Steps which occur at integer fractions of an energy $\left(E_{g}\right)$ suggest multi-photon ionization; excitation of electrons to the conduction band by absorbing several photons simultaneously $[5,6]$. In a pure 
multi-photon ionization model, as the photon energy increases, the damage threshold would exhibit an abrupt drop as the number of photons needed to bridge the gap (the order) decreases by one, and the transitions will take place at photon energies of $E_{g} / n$.

In wide band gap materials, excitons are prominent at room temperature which could potentially lower the energy required to promote an electron to the conduction band by $1 \mathrm{eV}$ or more [2]. For such a system, $E_{g}=\left(\varepsilon_{g}-\Delta\right)$ where $\Delta$ is the binding energy associated with an exciton.

The steps observed in figure 3.7 (located at $2.55 \mathrm{eV}$ and $3.9 \mathrm{eV}$ ) are both close to integer fractions of $7.8 \mathrm{eV}$, which is a likely location of the gap associated with the exciton peak in DKDP. The steps seen in figure 3.10 are close to $0.025 \mathrm{eV}$ in width, which is much narrower than a typical width of a large band gap exciton peak. Therefore, the width of the steps in the damage threshold data is more likely governed by the thermal distribution of electrons in the valence band.

The expected change of the damage threshold across a step can be estimated from representative multi-photon cross-sections $\left(\sigma^{(n)}\right)$ for third and fourth order absorption in wide band gap materials, which are on the order of $10^{-81} \mathrm{~cm}^{6} \mathrm{~s}^{2}$ and $10^{-114} \mathrm{~cm}^{8} \mathrm{~s}^{3}$ respectively $[5,7,8]$. The rate of electron absorption from a multi-photon process was given in equation 1.3

$$
\frac{d n_{e}}{d t}=N \sigma^{(m)} F^{m}
$$

where $\mathrm{N}, \sigma, \mathrm{m}$, and $\mathrm{F}$ are the density of active ions, the multi-photon crosssection, the order, and the photon flux density, respectively. By writing the photon flux density in terms of intensity and multiplying both sides of 1.3 by the 
band gap energy the rate of energy absorption can be written in the form of equation $3.1[7,9]$.

$$
\frac{d \varepsilon}{d t}=\frac{\varepsilon_{g} \cdot N}{(h c)^{n}} \sigma^{(n)} \cdot(I \lambda)^{n}
$$

where $\mathrm{I}$ is the laser intensity, $\mathrm{n}$ the absorption order and $\varepsilon_{\mathrm{g}}$ the band gap. For multi-photon absorption in an ideal (defect free) crystal the active ion density (N) would be close to the atomic density. The electron density $\left(\mathrm{N}_{\mathrm{e}}\right)$ necessary for rapid energy absorption would at most be the critical density $\left(N_{c}\right)$ (where the plasma becomes opaque) but could be several orders of magnitude less, depending on the size of the plasma. The plasma skin depth is give by equation 3.2

$$
\frac{c}{\omega_{p e}}=5.31 \times 10^{5} n_{e}{ }^{-\frac{1}{2}} \mathrm{~cm}
$$

where $c, \omega_{p e}$ and $n_{e}$ are the speed of light, electron plasma frequency, and electron density, respectively. The requirement of rapid energy absorption is then that the ratio of the plasma skin depth to size is close to 1 . Damage initiation sites (excluding cracks) from 3-ns laser pulses are generally on the order of $\sim 10$ times the size of the incident wavelength. Because the plasma skin depth varies inversely with the square root of the electron density, a plasma on the order of 10 times larger than the wavelength of the incident light suggests a lower limit of the necessary electron density of $0.01 \mathrm{~N}_{\mathrm{c}}[10]$.

Since the critical electron density differs insignificantly over the width of the steps in the data of figure 3.7, approximately the same amount of energy must first be absorbed through the multi-photon mechanism on either side, 
making the exact value of the band gap unimportant when estimating the step height. The intensity necessary for breakdown on each side of a step can be estimated by solving equation 3.1 for $\mathrm{I}_{\mathrm{c}}$.

$$
I_{c}=\sqrt[n]{N_{e} /\left(N \cdot \sigma^{(n)} \cdot \Delta t\right)} \cdot h c / \lambda
$$

For the step at $487 \mathrm{~nm}$ the critical density would be $5 \times 10^{21} \mathrm{~cm}^{-3}[10]$. The height of the step for a pure multi-photon mechanism may be estimated by computing the difference between the intensities necessary to produce the necessary density of electrons as the order of the multi-photon process changes. The predicted step at a change from 3 to 4 photon absorption is between 200 and $1500 \mathrm{~J} / \mathrm{cm}^{2}$ (for the lower and upper limits of $N_{e}$ of $0.01 N_{c}$ and $N_{c}$, respectively) rather than the observed value of $11 \mathrm{~J} / \mathrm{cm}^{2}$ at the $487 \mathrm{~nm}$ step.

The overall damage threshold for pure multi-photon absorption can be estimated as well. For example using equation 2, for $355-\mathrm{nm}$ radiation ( 3 photon absorption) the critical electron density of $10^{22} \mathrm{~cm}^{-3}$ suggests lower and upper bounds of the critical intensity of $I_{c}=3 \times 10^{10} \mathrm{~W} / \mathrm{cm}^{2}$ and $1.5 \times 10^{11} \mathrm{~W} / \mathrm{cm}^{2}$, respectively. The observed damage threshold is $\sim 3 \times 10^{9} \mathrm{~W} / \mathrm{cm}^{2}$. For the $3-\mathrm{ns}$ pulses used in this study, this corresponds to a predicted damage threshold ranging from $60 \mathrm{~J} / \mathrm{cm}^{2}$ to $450 \mathrm{~J} / \mathrm{cm}^{2}$ compared to the observed value of $10 \mathrm{~J} / \mathrm{cm}^{2}$. It is interesting to note that for very small spot sizes (for which a pure multiphoton mechanism would be expected to dominate), damage thresholds in excess of $200 \mathrm{~J} / \mathrm{cm}^{2}$ have been observed [11, 12]. The observed damage morphology is an additional indicator that pure multi-photon ionization is not the damage initiation mechanism (see figure 3.12) [6]. Multi-photon damage is 
localized at the peak of a Gaussian beam, while we observe the presence of discrete initiation sites situated throughout the beam.

Before the pure multi-photon model can be dismissed, it is necessary to consider the possibility of field enhancement by small particles. Surface plasmon resonance in small $(<40 \mathrm{~nm})$ particles has been shown to cause field enhancements of two orders of magnitude [13]. The field enhancement effect is sharply wavelength dependent and therefore would manifest itself as a narrow dip in the damage threshold for a single size of inclusion [14]. This model would require a uniform, continuous distribution of particle sizes to account for the wavelength dependence of the damage threshold similar to that observed in figure 3.7 by producing field enhancement at all wavelengths. However, particle size distributions are not, in general, uniform [15].

One of the first models which attempted to explain laser-induced damage is the "lucky" electron avalanche breakdown model. This model suggests that some electrons are present in the conduction band before the laser pulse [16]. The free electrons oscillate in the laser's fields and liberate additional electrons by impact ionization. Electrons would multiply in this manner until opaque plasma is generated, facilitating rapid absorption during the remainder of the laser pulse. The damage threshold would increase at frequencies large compared to the inverse of the electron collision time, because of reduced coupling to the lattice. Contrary to the observed data, the avalanche model predicts no dependence of the damage threshold on the laser wavelength until the laser frequency approaches the inverse of the electron collision time [7] . In 
addition Shen et al. have performed experiments which also suggest that any impact ionization mechanism would need in excess of $10^{18}$ seed electrons to be viable [17].

A more recent model proposed that foreign particles included in the crystal lattice during growth act as gray bodies, absorbing a fraction of all incident radiation. The absorbed energy heats the surrounding host material causing a collapse of the band gap and plasma formation [18]. Because this gray particle model predicts laser damage will be initiated at the site of particles included during growth, it is reasonable to expect damage to occur at discrete localized centers, and not necessarily at the peak energy density of the laser. This is in agreement with experimentally observed damage morphology in the 3 ns regime. For a uniform particle size distribution however, the gray particle model predicts smooth $1 / \lambda$ dependence for the damage threshold. This is roughly in agreement with the observed damage threshold data between the steps, but does not explain existence of the steps.

Extending the gray particle model by assuming two species of particles composed of materials with band gaps of $2.55 \mathrm{eV}$ and $3.90 \mathrm{eV}$ can provide a simple explanation that accounts for the steps in the damage threshold demonstrated in figure 3.7. A small sample of possible impurities are listed in table 3.1. 


\begin{tabular}{|c|c|c|}
\hline Impurity & $\begin{array}{c}\text { Concentration in } \\
\text { BD7 }\end{array}$ & $\begin{array}{c}\text { Band gap at } \\
\text { 300K[19] }\end{array}$ \\
\hline CdS & $\begin{array}{l}\text { Cd: } \quad \text { * } \\
\text { S: } \quad 2 \mathrm{ppm}\end{array}$ & $2.42 \mathrm{eV}$ \\
\hline $\mathrm{ZnO}$ & $\begin{array}{ll}\mathrm{Zn}: & 140 \mathrm{ppb} \\
\mathrm{O}: & \text { abundant }\end{array}$ & $3.2 \mathrm{eV}$ \\
\hline ZnS & $\begin{array}{ll}Z n: & 140 \mathrm{ppb} \\
\mathrm{S}: & 2 \mathrm{ppm}\end{array}$ & $3.6 \mathrm{eV}$ \\
\hline
\end{tabular}

Table 3.1

Potential impurities in DKDP with their band gaps and the concentration of their constituent elements in the solution used to grow the crystal. ${ }^{*} \mathrm{Cd}$ was not detected.

Although this is a possibility, the presence of such a coupled set of impurities is not supported by optical spectroscopy, transmission data, or EPR spectroscopy $[20,21]$.

In order to synthesize a model that accounts for lower than intrinsic damage threshold, the existence, size, and location of the steps as well as the observed damage morphology, we propose a defect assisted multi-step/photon mechanism. In this model, defect states in the gap alter the cross section for multi-photon absorption, in fact turning the process into a series of reduced order absorptions (see figure 3.13). 


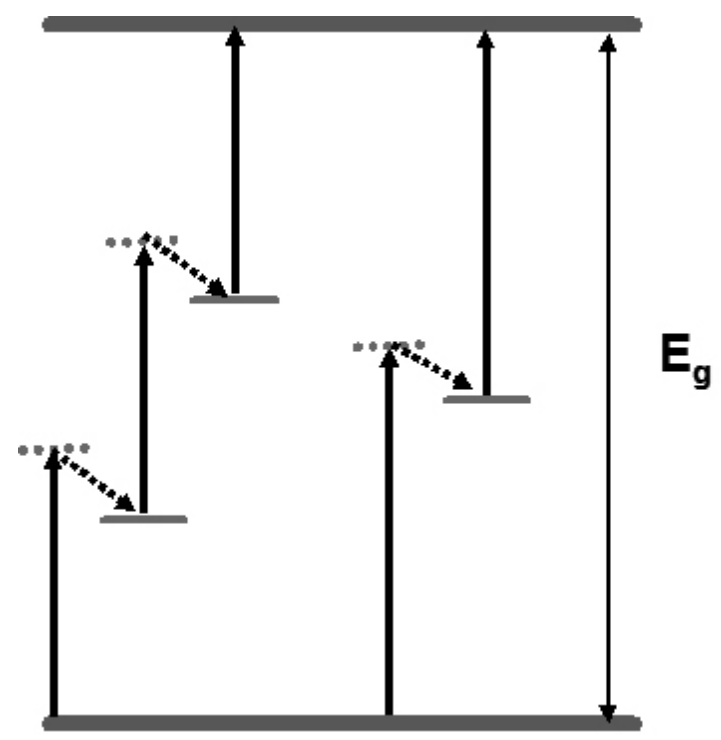

Figure 3.13

Individual photons with energies less than the band gap may be absorbed if electrons in the valence band can be excited into defect states within the band gap. This mechanism would allow photons to be absorbed in series rather than simultaneously, reducing the intensity (photon density) necessary to excite an electron from valence to conduction bands.

In the presence of intra-band defect states, excited state absorption can reduce the order of the multi-photon process needed for an electron to make a transition to the conduction band. Whether the mechanism is a series of single photon absorptions, or a mixture of single and multi-photon absorptions, this model accounts for the sharp steps in the damage threshold. The number of photons needed to create a free electron would be very sensitive to changes in photon energy near Eg/n. From this we would expect the next step $(n=4)$ in the damage threshold at or near $635 \mathrm{~nm}$. 


\subsection{References}

[1] Palik, E.D., Handbook of optical constants of solids (Academic Press, Orlando, 1985).

[2] Fox, M., Optical Properties of Solids (Oxford University Press, 2001).

[3] Ogorodnikov, I.N., et al., Optika i Spektroskopiya 91, 243-51 (2001).

[4] Liu, C.S., et al., Phys Rev Lett 9101, 5505 (2003).

[5] Nathan, V., et al., J. Opt. Soc. Am. B, Opt. Phys. (USA) 2, 294-316 (1985).

[6] Stuart, B.C., et al., Phys. Rev. B, Condens. Matter (USA) 53, 1749-61 (1996).

[7] Jones, S.C., et al., Optical Engineering 28, 1039-1068 (1989).

[8] Gorling, C., et al., Appl Phys B-Lasers Opt 74, 259-265 (2002).

[9] Schmid, A., et al., Phys. Rev. B, Solid State (USA) 16, 4569-82 (1977).

[10] Chen, F.F., Introduction to plasma physics and controlled fusion (:Plenum Press, New York, 1984).

[11] Yoshida, H., et al., Applied Physics B-Lasers and Optics 70, 195-201 (2000).

[12] Oconnell, R.M., Appl. Opt. (USA) 31, 4143-4153 (1992).

[13] Kottmann, J.P., et al., Appl Phys B-Lasers Opt 73, 299-304 (2001).

[14] Kneipp, K., et al., Chem Rev 99, 2957-2975,COVER4 (1999).

[15] Risovic, D., Appl. Opt. (USA) 41, 7092-7101 (2002).

[16] Bloembergen, N., IEEE J. Quantum Electron. (USA) QE-10, 375-86 (1974).

[17] Shen, X.A., et al., Phys Rev Lett 62, 2711-13 (1989).

[18] Feit, M.D., et al., SPIE, 1997. 2966: p. 417.

[19] Kittel, C., Introduction to solid state physics (Wiley, New York, 1996).

[20] Demos, S.G., et al., J. Appl. Phys. (USA) 85, 3988 (1999).

[21] Garces, N.Y., et al., J. Appl. Phys. (USA) 89, 47-52 (2001). 


\section{Chapter 4}

\section{Experimental Technique for In-Situ Dynamic Temperature Measurements during Laser-Induced}

\section{Breakdown}

\subsection{Introduction}

In order to study the emission which accompanies laser-induced breakdown in optical materials, several constraints must be observed. The collection and relay optics must function over entire spectral range of the emission, a method for dispersing the signal spectrally and gating it temporally must be employed, and a sensor with enough sensitivity to record the relatively weak plasma flash is needed. As the scattered light from laser is many orders of magnitude brighter than the plasma emission, a method for removing the scattered laser light must also be devised. 


\section{a. ICCD Physics}

Intensified charged coupled devices (ICCD) have the advantages of photo-multiplier tubes and charge coupled devices combined in one instrument. As an ICCD can also be time gated, it makes an ideal sensor for this experiment. In an ICCD, incident light strikes a photocathode, producing electrons which are then accelerated by an electric field. When the high energy electrons impact a fluorescent screen, many photons are produced for each incident electron (see figure 4.1). A CCD records the image consisting of these photons. This intensified image is up to $10^{4}$ times brighter than an un-intensified image.

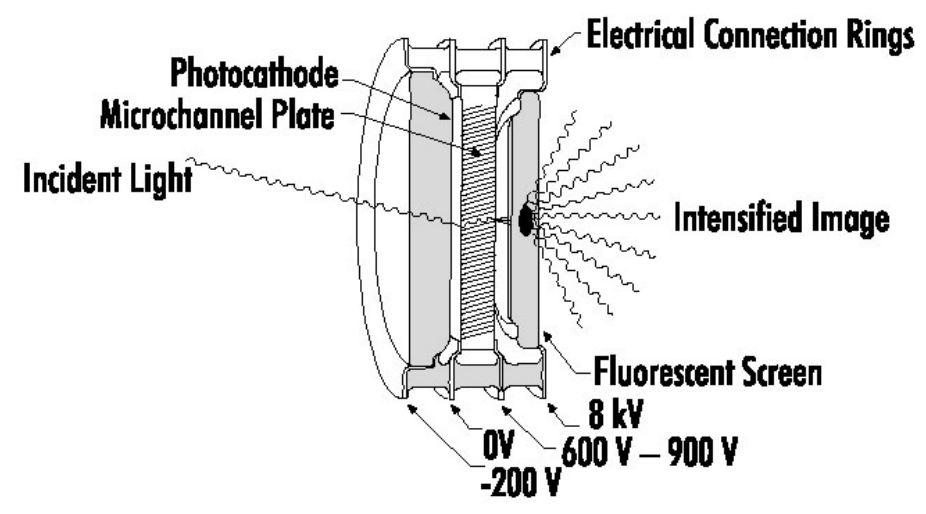

Figure 4.1

A schematic of a Roper Scientific ICCD camera similar to the one used in these experiments. A CCD array records the intensified image. 


\section{b. Limitations of refractive optics}

The utility of refractive optics is limited both by the wavelengths for which they transmit, and by chromatic adoration. Optics fabricated from BK7 (the most common type of optical glass) are limited to use at wavelengths above $\sim 300 \mathrm{~nm}$. Fused silica and $\mathrm{CaF}_{2}$ optics can be used to $180 \mathrm{~nm}$ and $110 \mathrm{~nm}$ respectively, however both will produce chromatic aberration in an optical path. Figure 4.2 shows the calculated focal spots from a fused silica lens and an off-axis parabolic mirror.

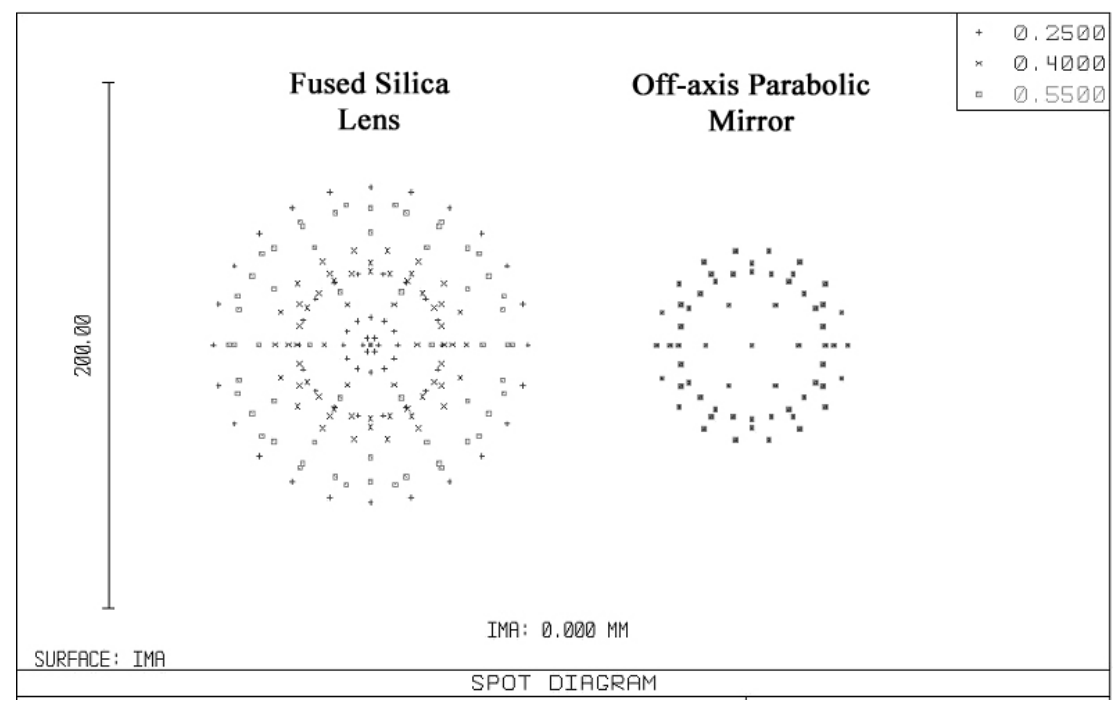

Figure 4.2

The focal spots produced by a fused silica lens and an off-axis parabolic mirror when focusing a broadband light source. Because the focal length of the lens is slightly different for each wavelength the tightest focal spot is nearly double that produced by the mirror.

Although achromatic lenses remove chromatic aberration for two separated wavelengths, the optical glass used in their construction suffers from similar UV transmission limitations to BK7. 


\section{c. Off-axis parabolic mirrors}

The use of reflective optics provides a solution to both chromatic adoration and transmission difficulties encountered with refractive optics. Off-axis parabolic mirrors (OAPMs) can be described as a cylinder with a parabolic section removed (see figure 4.2). The resulting surface is made highly reflective at the wavelengths for which the mirror will be used. Because of the complex geometry of these mirrors, they are generally constructed either from machined metal or from metal deposited onto a machined substrate. As with refractive optics (lenses), an OAPM will focus outgoing rays at a distance from the mirror equal to its focal length if the incident ray is collimated. Unlike refractive optics, OAPMs operate with a defined angle between incident and reflected rays which ranges from between 0 to 90 degrees depending on the geometry of the mirror.

By using OAPMs for both focusing and collection optics, several benefits are realized. Foremost is a nearly flat reflection spectrum over the entire region of the experiment. Second is the elimination of chromatic aberration because, unlike a refractive lens, the OAPM has the same focal length for all wavelengths. 


\section{d. Removing the laser line}

The plasma emission is largely within the spectral window from $200 \mathrm{~nm}$ to $600 \mathrm{~nm}$. During experiments using pump wavelengths of $355 \mathrm{~nm}$ or $532 \mathrm{~nm}$, care must be taken to prevent damage to the ICCD. Direct exposure to laser light will at least bleach, if not burn the camera. The typical method for blocking a laser line is by use of a holographic notch filter. The spectrum of a $355-\mathrm{nm}$ notch filter can be seen in figure 4.3. The spectrum clearly demonstrates that the $355 \mathrm{~nm}$ light will be attenuated, but light below $300 \mathrm{~nm}$ will also be lost. As the UV portions of the spectra are of great importance to fitting the Planckian distribution (as discussed below), this technique is not acceptable.

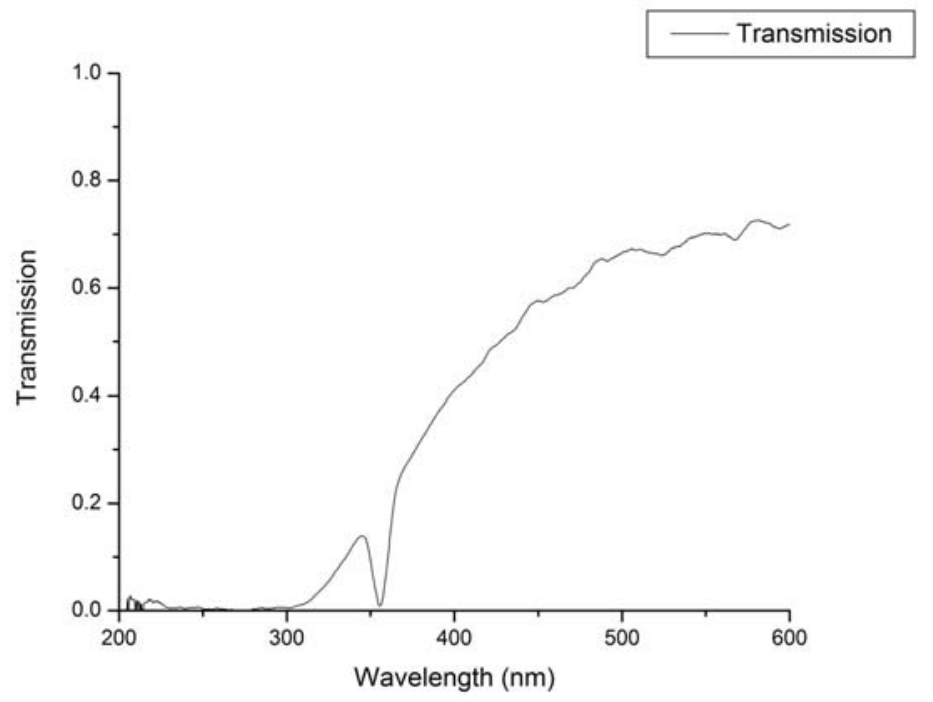

Figure 4.3

Transmission spectrum of a 355-nm notch filter. The spectrum shows that the filter will block a 355-nm laser line, but only at the cost of losing the light below $300 \mathrm{~nm}$.

The alternate technique used in this experiment was to place a physical obstruction over the pixels of the ICCD where the laser line would be incident. 
Except for diffraction at the edge of the obstruction this technique produces a step function response. In other words transmission is 1 except near $355 \mathrm{~nm}$ where it is $\sim 0$. The limitation of this method is that a $100 \mathrm{~nm}$ gap is produced in the spectra. Losing such a section of the middle of the spectra still allows fits to be well constrained by the data on either side of the gap, allowing for accurate fits to the Planckian distribution. 


\subsection{Experimental methods and techniques}

\section{a. Apparatus}

Within these design constraints, the experiment was conducted as follows: A $\sim 5 \mathrm{~mm}$ diameter pump beam of $355 \mathrm{~nm}, 532 \mathrm{~nm}$, or $1064 \mathrm{~nm}$ is reflected off a small turning mirror (referred to as the injection mirror) into an off-axis parabolic mirror which focuses the beam to a spot size of $\omega_{0} \sim 50 \mu \mathrm{m}$ into the bulk of a sample. The plasma formed by the breakdown of the sample produces a broadband emission. Approximately $3 / 8$ of the emission is collected by the same mirror which focused the pump beam. Because the plasma is formed at the focus of the mirror, the returning emission is collimated into a 3 " diameter beam counter-propagating with the pump beam. When the emission beam arrives at the injection mirror all but approximately $1 / 9$ of the beam bypasses the mirror (see figure 4.4). The remaining emission is then focused by a second off-axis parabolic mirror into the slit of a spectrometer. Because the ratio of the two focal lengths is $1: 4$, a slit width of 200 microns will allow spectra to be collected from 50 microns diameter region. By matching the collection region to the spot size of the pump beam (also $\sim 50 \mu \mathrm{m}$ in diameter) the signal can be maximized without collecting any extraneous light from outside the damage region.

The spot size of the pump beam can be regulated by altering the diameter of the input beam with a telescope. The spot size achieved is governed by equation 4.1

$$
\omega_{0}=\frac{1.44 \cdot f \cdot \lambda}{D}
$$


where $\omega_{0}$ is the spot size, $f$ is the focal length of the mirror, $\lambda$ is the wavelength of the light and $D$ is the diameter is the input beam. Although the focal length of the mirror remains constant for all wavelengths the spot size, if not corrected, will vary linearly with $\lambda$.

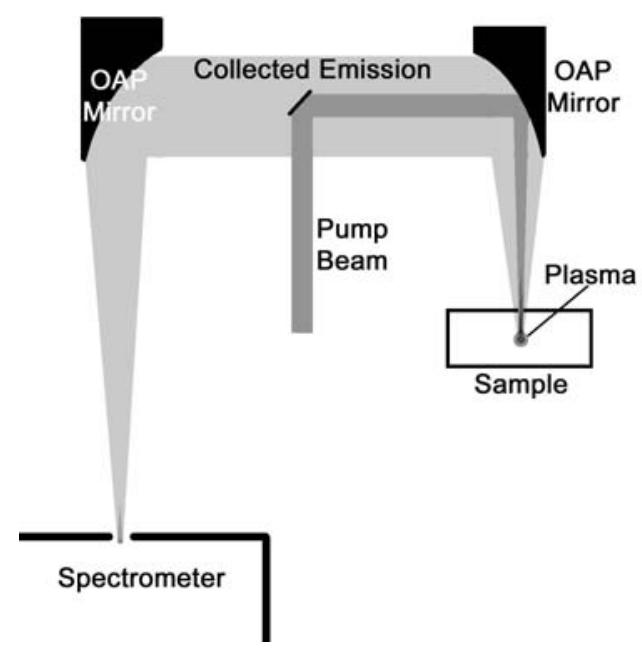

Figure 4.4

A schematic diagram of the apparatus. A pump beam is introduced into the system by a small turning mirror. The pump beam is focused by an off-axis parabolic mirror into the bulk of a sample. The plasma produced emits broadband radiation of which a fraction is collected by the off-axis parabolic mirror. This emission is collimated by the mirror into a 3" beam counterpropagating with the pump beam. Because the emission beam overfills the small turning mirror, only $16 \%$ of the emission is reflected out of the beam. The remaining emission continues past the turning mirror and is incident on a second off-axis parabolic mirror. This second mirror focuses the emission beam onto the slit of a spectrometer. 


\section{b. Alignment}

The alignment of an off-axis parabolic mirror is not a trivial exercise. Six degrees of freedom must be adjusted, compared to only 3 for a standard mirror. In other words, alignment of a flat mirror depends only on the location where it intercepts an incident beam and two steering angles. In addition to these parameters, an OAPM is sensitive to where on the mirror the incident beam hits (both vertically and horizontally) and also the rotation of the OAPM about the axis of the incident beam. There are methods in the literature describing ways to align OAPM by using 3 alignment beams propagating in parallel [1].

In practice, it has proved much less time consuming to use a more simple method developed during the course of these experiments. To align the mirror, the optical axis of both the incident and reflected beams of the OAPM must be denoted. The optical axis is most easily established by temporarily replacing the OAPM with a flat mirror and fixing the angle between the incident and reflected beams to that appropriate to the OAPM. Second, (assuming that the incident beam is already aligned parallel to the table), the flat mirror must be adjusted so that the reflected beam does not change height as it propagates. Once these two parameters are set, the optical axis is demarcated by the reflected beam. Fiducials such as crosshairs or pinholes should be placed to mark the beam. The first fiducial should be as close as possible to the flat mirror without interfering with the OAPM when it is replaced. The second fiducial should be

placed about 4 to 5 times the focal length of the OAPM from the first fiducial. The further apart the fiducials are, the more precise the alignment will be. Once 
the OAPM is reinstalled however, the beam will have passed through a focus and will be diverging when it reaches the second fiducial. Therefore, it is necessary to keep the spacing between fiducials small enough that the beam is not overlarge at the second fiducial.

After the placement of the fiducials is complete, the flat mirror should be replaced with the OAPM. By insuring that the OAPM intercepts the incident beam in the same location as the flat mirror and by using a bubble level (or similar device) to remove any vertical tilt, two of the 6 parameters can be eliminated. Vertical alignment of the OAPM is obtained by adjusting the vertical position until the reflected beam is on the first fiducial and then rotating the OAPM (about the axis of the incident beam) to put the reflected beam on the second fiducial. These steps are repeated until the reflected beam is simultaneously on (vertically) both fiducials. To align the horizontal axis, the same procedure is repeated, except now the horizontal translation of the OAPM is adjusted with the horizontal tilt. If the focus of the OAPM does not exhibit any astigmatism, then it is well aligned. 


\section{c. Calibration}

\section{i. Wavelength}

After first selecting the appropriate grating for the wavelength range of interest, the spectrometer must be calibrated for wavelength. The grating position must be adjusted so that the wavelength range of interest is incident on the ICCD. This is most easily accomplished by the use of a calibration lamp which produces a spectrum of known atomic lines. For the 200 to $600 \mathrm{~nm}$ wavelength range, $\mathrm{HgNe}$ lamps are ideal (see figure 4.5).

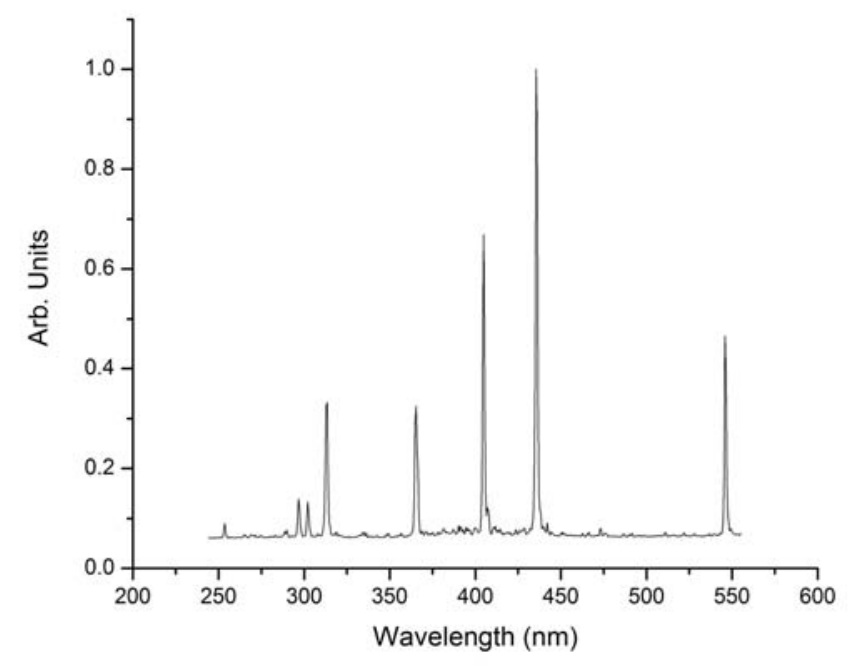

Figure 4.5

Atomic line spectrum from a $\mathrm{HgNe}$ lamp. A $260-\mathrm{nm}$ long pass filter is used to attenuate the $250 \mathrm{~nm}$ line.

The $250-\mathrm{nm}$ line is so much more intense than the other lines that it is easily identifiable. Care must be taken to insure that the signal is not due to higher order reflections. After adjusting the grating position, the wavelength values must be assigned to the various pixels of the ICCD. By identifying atomic lines 
and establishing the wavelength at two or more widely dispersed pixels the wavelength associated with any pixel can be determined by interpolating or extrapolating. A $260-\mathrm{nm}$ long pass filter (or similar) must be used to reduce the intensity of the 250-nm line. This allows the integration time on the ICCD to be increased to the point that the other atomic lines are visible without causing bleaching from the far more intense $250-\mathrm{nm}$ line. 


\section{ii. Intensity}

Once the ICCD is calibrated for wavelength, the nature of this experiment requires the system to be calibrated for intensity as well. This calibration enables the counts recorded by the ICCD to be interpreted in terms of $\mathrm{W} \mathrm{cm}^{-2} \mathrm{~nm}^{-1}$, which is essential in fitting the collected data to the Planckian distribution so that the temperature of the plasma may be measured. The system response was calibrated by placing a $50-\mu \mathrm{m}$ pinhole at the focus of the first off-axis parabolic mirror and illuminating it sequentially with a standard blackbody light source and a standard calibration deuterium lamp (see figure 4.6). The UV end of the $D_{2}$ lamp was attenuated by a $225-\mathrm{nm}$ long pass filter to prevent artifacts which are produced by intense light illuminating the edge of a CCD chip. The pinhole size is nearly identical to the focal spot size. This configuration tightly controlled the collection geometry and allowed the system to be calibrated in absolute units by dividing the data collected from the calibration lamp by the actual output of the lamp (provided by the manufacturer by comparing the output of the lamp to a National Institute of Standards and Technology NIST traceable standard). 


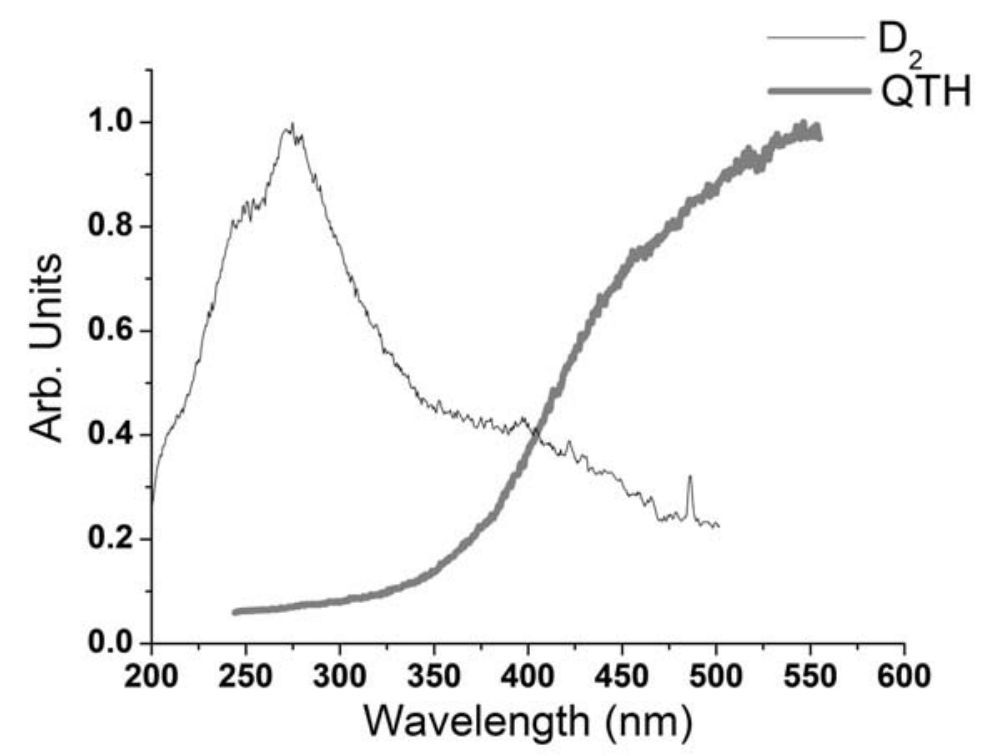

Figure 4.6

The spectra produced by the QTH (blackbody) lamp and the $D_{2}$ arc lamp. The UV end of the D2 lamp is clipped by a 225-nm long pass filter.

The calibration curve derived by comparing both lamps' measured spectra to their actual spectra is displayed in figure 4.7. As the light from the lamps traveled though the entire optical path of the experiment, all response from any of the optics is also removed from the data by the calibration curve. 


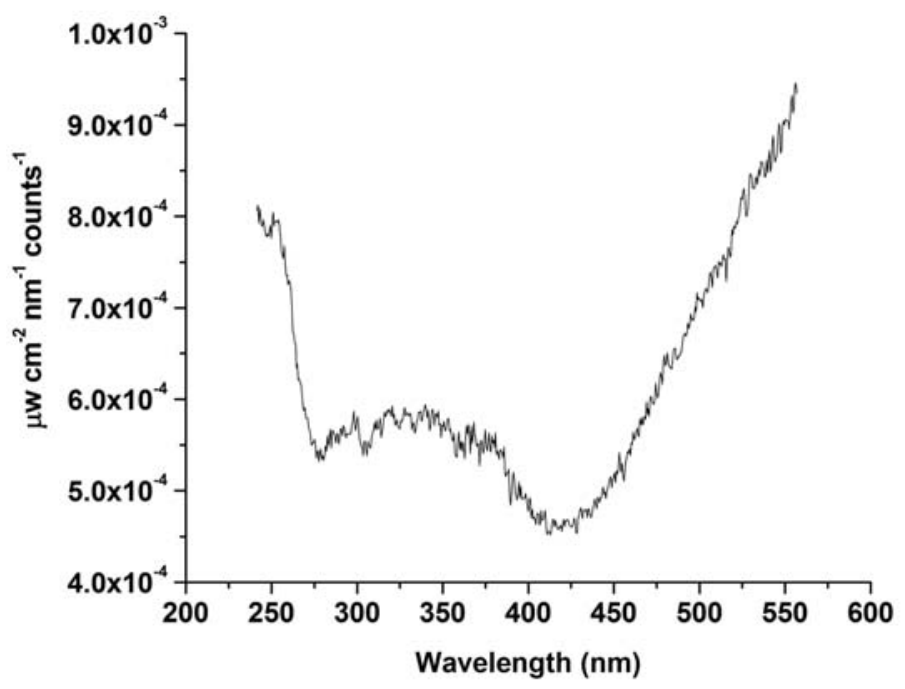

Figure 4.7

The calibration curve which converts the counts collected by the ICCD into absolute units. The curve represents the combined effects of non uniform transmission from optics, quantum efficiency of the ICCD, and f number of the collecting mirror. 


\section{iii. Fluence}

The final calibration necessary for the experiment is the determination of the fluence of the pump beam. This is accomplished by performing a knife edge measurement along both the vertical and horizontal directions. A knife edge measurement is performed by monitoring the energy in the beam before and after the focus while a blade is slowly moved to obstruct the beam (see figure 4.8).

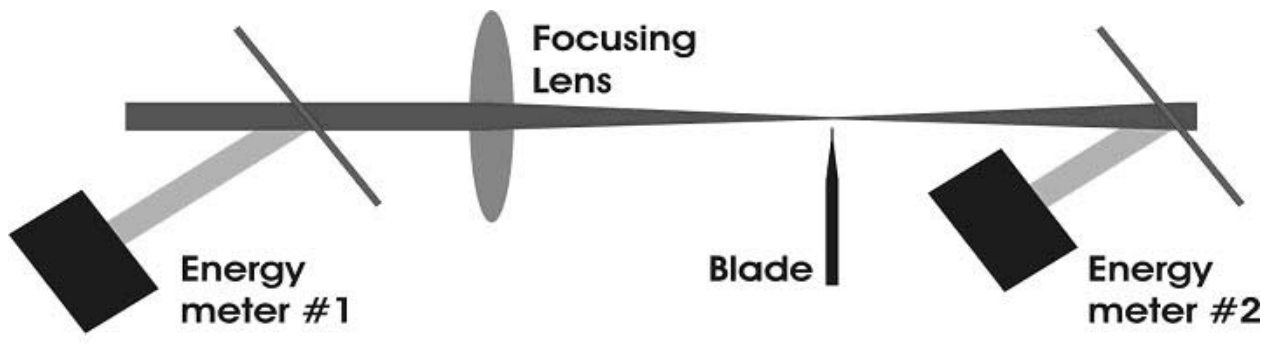

Figure 4.8

Schematic representation of a knife edge measurement. Incident and transmitted energy is monitored, with the difference attributed to obscuration by the blade. Plotting the derivative of transmitted energy vs. blade position indicates the profile of the beam where it was cut by the blade.

By plotting the derivative of the transmitted energy with respect to blade position as a function of blade position, the size and features of the beam at the location of the cut are determined (see figures $4.9 \mathrm{a \& b}$ ). There are two simple methods which may be employed to ensure that the cut is made at the waist of the beam. The blade can be translated through the focal point with the beam incident on its side. When the blade nears the focal point (the location of the waist), the metal of the blade will begin to breakdown producing a visible spark and an audible snap if the energy of the beam is high enough. By translating the 
beam to the position with the most intense spark/snap and then reducing the energy in the beam and repeating, the waist of a sharply focusing beam can be quickly and accurately located. An alternate technique is to simply make many measurements of the beam diameter at different locations along the beams axis of propagation.

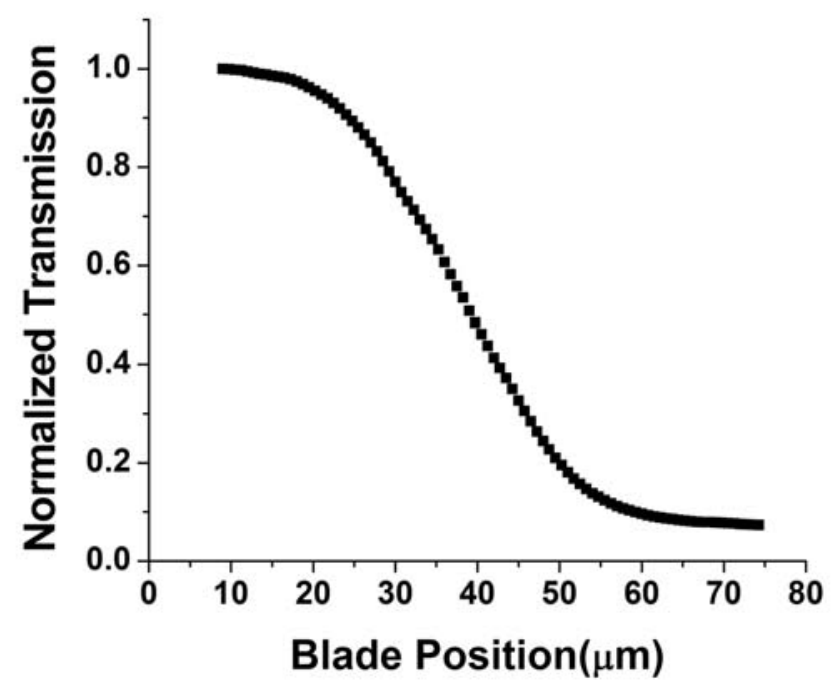

Figure 4.9 a

Transmission vs. blade position data taken during a knife edge measurement. 


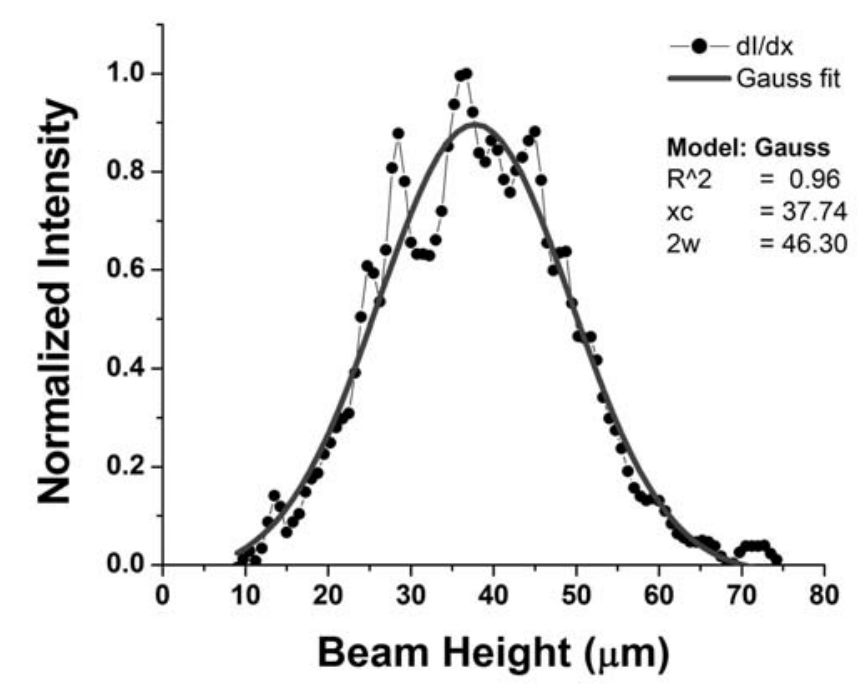

Figure $4.9 \mathrm{~b}$

The derivative of the data in figure 4.9a. The data show the profile of the beam in one dimension. A Gaussian fit gives the spot size as $46.3 \mu \mathrm{m}$.

The benefit of this technique is that the entire focal geometry of the beam can be measured. This establishes the Raleigh range and also determines if any astigmatism is present, which would indicate imperfect alignment of the OAPM.

The knife edge measurement is in theory simple, accurate, and inexpensive. A key advantage to the method is that the resolution of the measurement is determined by the step size for the motion of the blade cuting through the beam. This enables the knife edge measurement to evaluate beams too small for a CCD camera to measure. In practice, the method is simple, accurate, and arduous, especially if the second method discussed above is used to find the beam waist. In order to make the method more practical to use, an automated apparatus was built using a Newport ESP 300 stage and three $850 \mathrm{G}$ stage actuators. Labview code was written to perform multiple measurements unattended (see figure 4.10). The code allowed the user to select the number 
and spacing of the measurements to be made, as well as to set the resolution for each measurement. The minimum resolution was $0.5 \mu \mathrm{m}$ step size. For comparison, the best CCD camera available at the time of these experiments had a resolution of $5 \mu \mathrm{m}$.

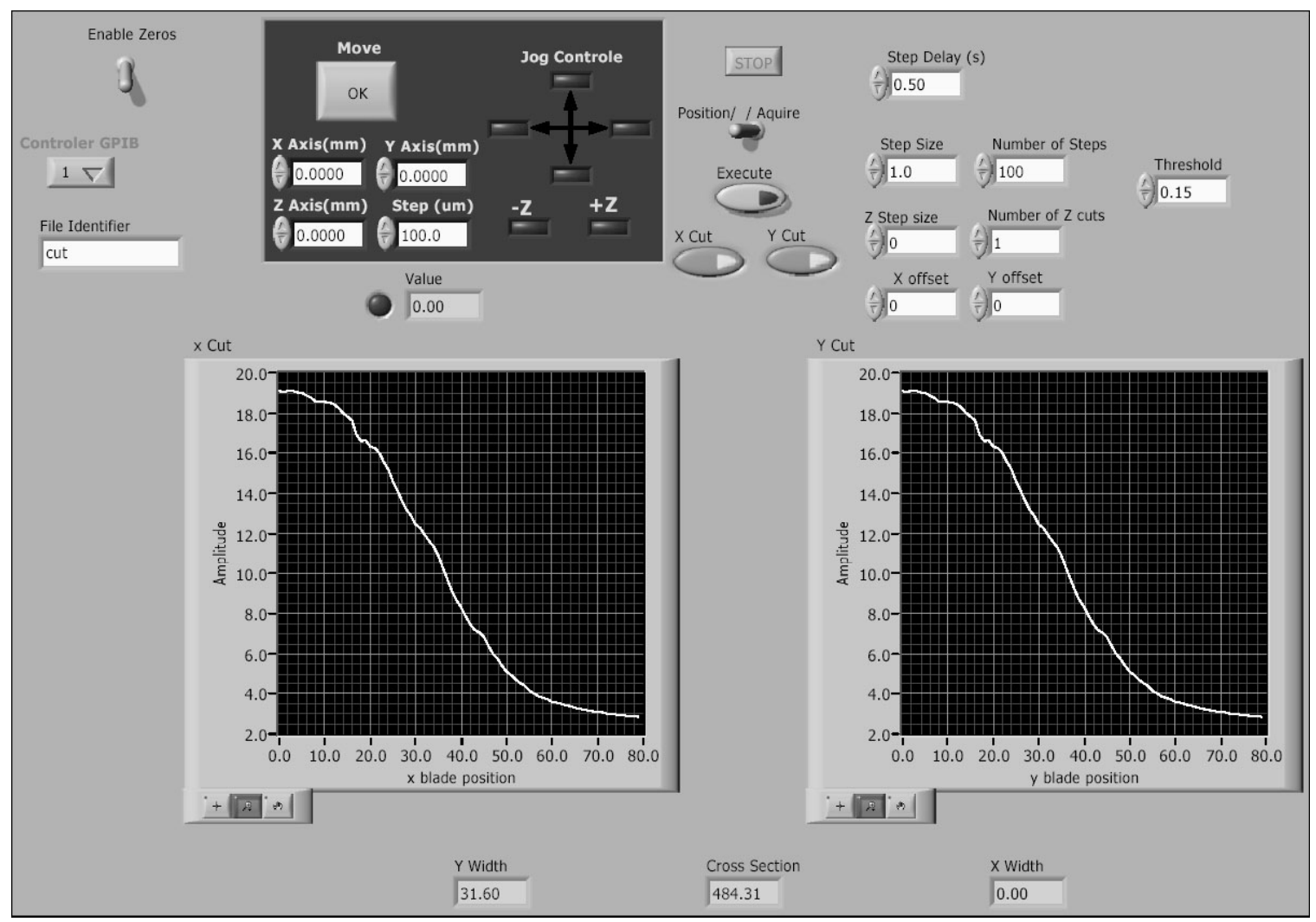

Figure 4.10

The front panel of the automated knife edge measurement program. 


\subsection{Data acquisition}

Once the lengthy alignment and calibration procedures are complete, data acquisition becomes a relatively simple matter. Other than the sample and its position, there are only three parameters to be monitored and/or adjusted during an experiment. The width of the front entrance slit to the spectrometer is generally set to $200 \mu \mathrm{m}$, unless the signal is large enough to saturate the ICCD. The other two parameters are the delay and integration times for the ICCD. These control the time relative to the laser pulse when the ICCD records spectra and for how long, respectively. The minimum integration time is $5 \mathrm{~ns}$ and the minimum increment in the delay time is $1 \mathrm{~ns}$, although there is about $1 \mathrm{~ns}$ of jitter. The spectra collected at any given delay were highly repeatable. Several spectra were collected at each delay time and averaged. Data was collected for delay times ranging from before the pulse up to $100 \mathrm{~ns}$ for all samples, and up to $50 \mu \mathrm{s}$ for fused silica. A sample of calibrated data is shown in figure 4.11. 


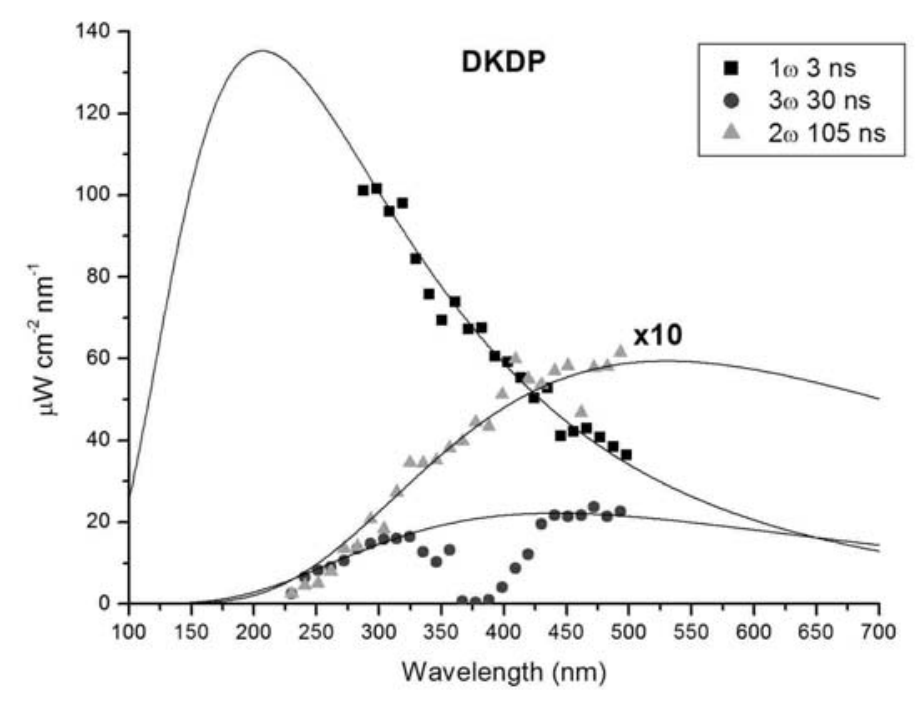

Figure 4.11

The individual symbols are representetive points from the calibrated spectra from DKDP. The solid curves through the data are the best fits to a Planckian distribution (equation 4.2). The legend indicates the excitation wavelength and the delay time for which the data were acquired. The $2 \omega 105 \mathrm{~ns}$ data and fit has been multiplied by a factor of 10 to enhance its visibility.

Once the spectra are collected and reduced using the calibration techniques discussed above, they are fitted to the Planckian distribution

$$
I(\lambda)=\varepsilon_{e} \cdot 8 \cdot \pi \cdot c \cdot h \cdot \lambda^{-5}\left(e^{h c / k T \lambda}-1\right)^{-1}
$$

where h, c, and k are Planck's constant, the speed of light, and Boltzmann's constant respectively. Here $\varepsilon_{\mathrm{e}}$ and $\mathrm{T}$ are fitting parameters for the effective emissivity and temperature respectively. The fits to the experimentally measured spectra in figure 4.11 are shown as solid lines.

The good fits of the experimental data to the Planckian distribution allow for the extraction of the temperatures of the plasma. Figure 4.12 shows the plasma temperature as a function of the delay time in $\mathrm{SiO}_{2}$ under irradiation at 1064-nm, 532-nm and, 355-nm. The overall magnitude of the signal provides 
information about the effective emissivity $\varepsilon \pi r^{2}$, where $\varepsilon$ is the emissivity and $r$ is the radius of the ionized region. It is necessary to consider the effective emissivity because both the radius of the ionized region and its emissivity are unknown. If an emissivity of unity is assumed, then the diameter of the ionized region is estimated to be on the order of $40 \mu \mathrm{m}$ to $60 \mu \mathrm{m}$ by comparing the measured spectrum to that of an ideal blackbody at the same temperature collected though a pinhole of known dimensions. From this size and the observed temperature in fused silica of $12000 \mathrm{~K}$, we can use the equation of state for quartz to estimate that a minimum of $12 \%$ of the laser pulse energy must be absorbed [2].

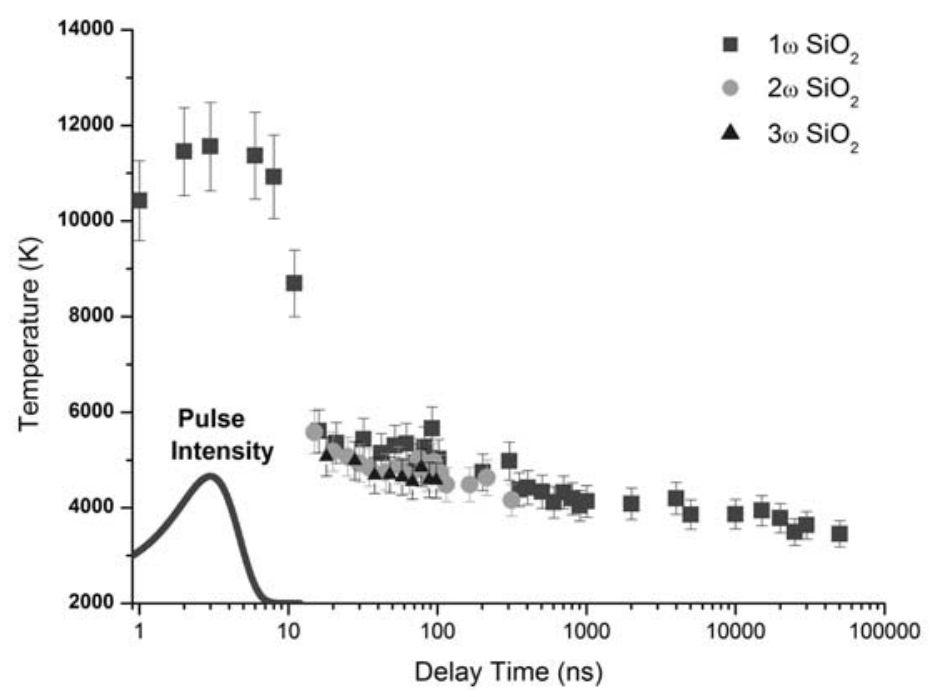

Figure 4.12

Fitted plasma temperatures for fused silica data. The temperatures plotted as a function of delay time as extracted from the fit to equation 1 of the time-gated emission spectra under $1064-\mathrm{nm}, 532-\mathrm{nm}$, and 355-nm excitation. A schematic representation of the 1064-nm pulse is shown in arbitrary units. 


\section{$4.4 \quad$ References}

[1] Bekhtina, S.V., et al., Sov J Opt T 59, 611-613 (1992).

[2] T4_Group, SESAME Report on the Los Alamos Equation of State Library. 1983: Los Alamos. 


\section{Chapter 5}

\section{Determining the Local Values of Temperature during Laser-Induced Damage in Wide Gap Materials}

\subsection{Introduction}

Laser-induced damage in wide band-gap optical materials is the result of material modifications arising from extreme conditions occurring during this process. The material absorbs energy from the laser pulse and produces an ionized region that gives rise to broadband emission. By performing a timeresolved investigation of this emission, we demonstrate both that it is blackbody in nature and provide the first direct measurement of the localized temperature of the material during and following laser damage initiation for various optical materials. 
An illustration of the radiation accompanying the breakdown process (also referred to laser-induced damage, or LID) may be seen in figure 5.1.

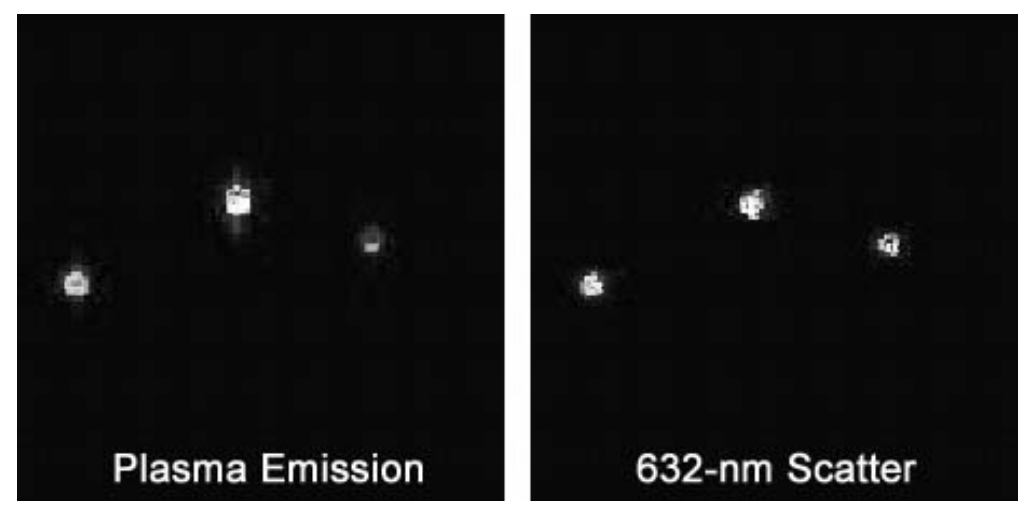

Figure 5.1

The image on the left shows the emission produced by the plasma accompanying the laser-induced breakdown at each of three sites. The damage was induced by a pulsed $355 \mathrm{~nm}$ laser focused with a cylindrical lens. The damaging beam overfills the image and is propagating left to right. The image was acquired with a CCD camera equipped with a long pass filter to remove both scattered laser light and Raman shifted light. On the right, a scattering image taken using a cw HeNe laser for illumination 10 seconds after the $355-\mathrm{nm}$ pulse is terminated. Sites in the right hand image are visible because the host matrix has been permanently modified by the breakdown event (laser damage). Discontinuity in material composition scatters the HeNe light by 90 degrees (into the CCD camera). The damage sites evident in the right hand image are consistent with the observation that whenever laser damage occurs it is accompanied by plasma emission.

This emission universally accompanies laser-induced damage, which makes understanding its nature important to understanding the underlying physical processes. The time gated spectroscopic measurements of this emission (acquired by the methods discussed in the previous chapter) demonstrated that it is blackbody in nature. Because the spectra are blackbody in nature (i.e. conform to the Planckian distribution), the temperature of the plasma may be determined from the spectra. Here the universal nature and the implications of the phenomenon will be considered. 
Various works in the past have demonstrated that it is possible to obtain blackbody emission during a laser damage event [1-10]. Although most of these observations were from damage initiated on a surface (often confined by a liquid), two works in particular have examined the emission from laser damage events in bulk material. Gorbonov and Maksimuk reported that the emission which accompanies laser-induced damage in the bulk of $\mathrm{NaCl}$ is blackbody in nature microseconds after damage. They reported that the spectra collected by integrating from the laser damage event out to $1 \mu$ did not appear to be blackbody (see figure 5.2).

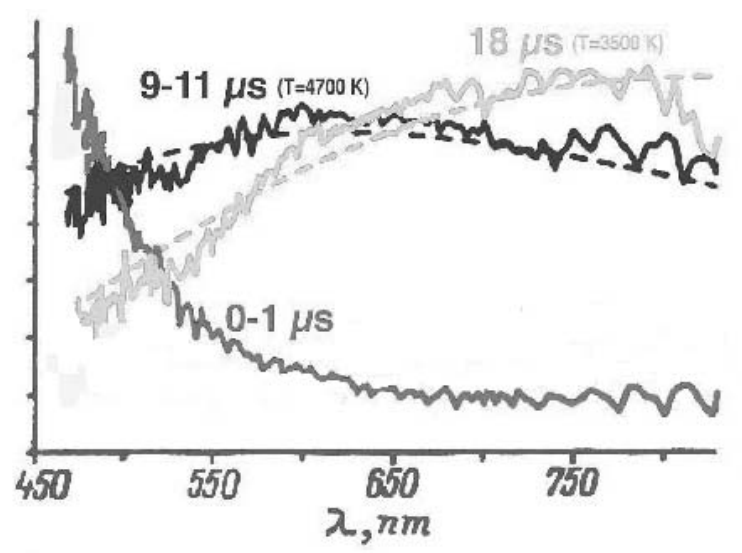

Figure 5.2

From Gorbunov, A.V. and M. Maksimuk, Fiz Tverd Tela, 1994. 36(5): p. 1429-36 The spectra of plasma emission accompanying breakdown in $\mathrm{NaCl}$ crystals.

The non-thermal nature of the spectra is consistent with cooling on a faster time scale than the $1 \mu$ s integration time used in the experiment.

More recently, work performed by Bandis et al. collected spectra with ns temporal resolution. This investigation of the plasma emissions was performed in $\mathrm{NaNO}_{3}$ which does not transmit below $300 \mathrm{~nm}$ [11]. Bandis and co-workers observed that the spectra were broadband and concluded they were caused by 
emission from laser-induced plasma. Because of the relatively long wavelength at which the $\mathrm{NaNO}_{3}$ cut off the emission, and the fact that they were looking at time in the ns regime, they did not observe the full nature of the spectra distribution (see figure 5.3).

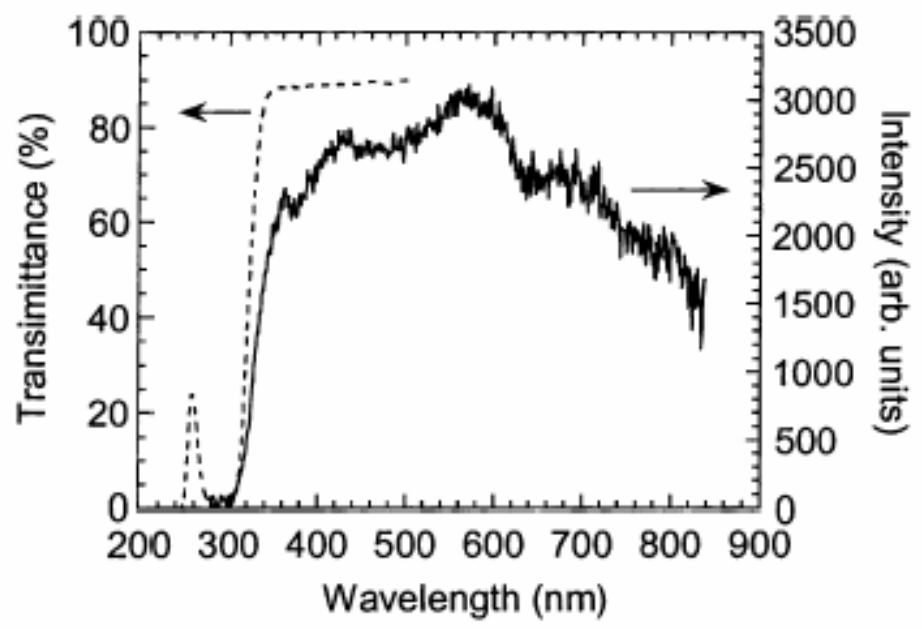

Figure 5.3

From Bandis et al. / Applied Surface Science 197-198 (2002) 100-106 "Spectrum of the visible radiation emitted from a $\mathrm{NaNO}_{3}$ bulk emission sites upon exposure to1064 $\mathrm{nm}$ laser radiation with fluence near the single pulse damage threshold. The absence of emission at wavelengths lower than $350 \mathrm{~nm}$ is consistent with absorption from the crystal surrounding the emission sites. Also note the absence of any sharp peaks due to atomic emission"

These issues have been avoided in this work by using a data acquisition system with a temporal resolution faster than the cooling rate of the plasma and by performing measurements on a variety of samples, the majority of which transmit deep into the UV. 


\subsection{Collected data}

\section{a. Collection parameters}

Spectra accompanying damage induced by both $355-\mathrm{nm}, 532-\mathrm{nm}$ and 1064-nm radiation was measured. Figure 5.4 shows uncalibrated data recorded during a $355-\mathrm{nm}$ damage event. The dip in the spectra from 325 to 425 is caused by the obstruction (discussed in the previous chapter) used to attenuate the laser line. This feature is common to all data taken during $355-\mathrm{nm}$ damage events and is disregarded during the fitting of the spectra to the Planckian distribution. The laser line is excluded in 532-nm experiments simply by adjusting the grating to steer it off the edge of the ICCD. Because the 1064-nm line is far from the spectral region of interest, experiments using these wavelengths give the least constrained operating conditions, both temporally, as well as spectrally. In Contrast both 355-nm and 532-nm experiments, images may not be taken within $15 \mathrm{~ns}$ of the laser pulse. The reason for this is illustrated by the spike in the data of figure 5.4 at $355 \mathrm{~nm}$ still visible $30 \mathrm{~ns}$ after the peak of the laser pulse. At delay times near the laser pulse, bleaching of the ICCD from scattered laser light is encountered. 


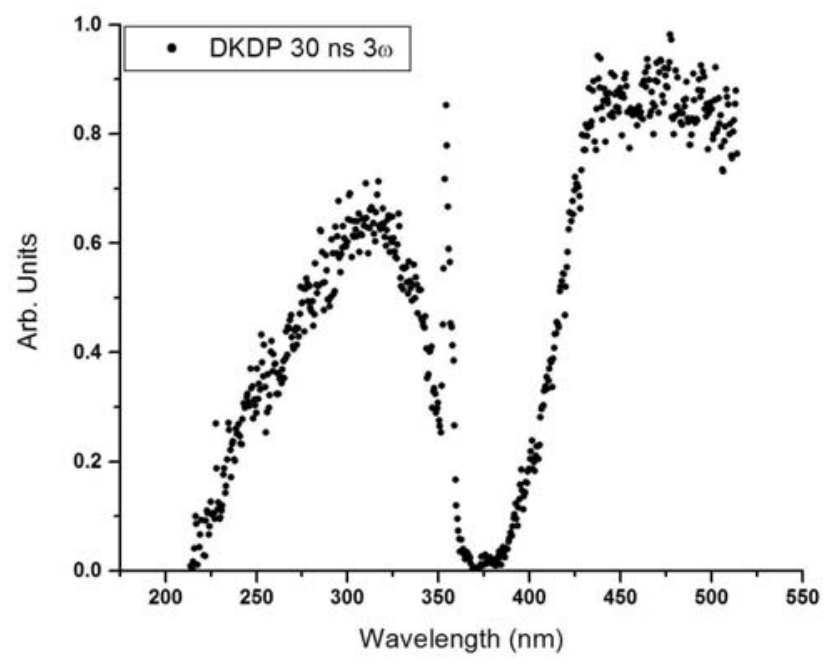

Figure 5.4

Uncalibrated data taken in DKDP $30 \mathrm{~ns}$ after the peak of the 355-nm damaging pulse. The dip in the spectra is produced by the obstruction used to attenuate the 355-nm laser line. Despite the obstruction some laser light still incidents on the ICCD, precluding image acquisition during and immediately after the pulse. 


\section{b. Samples}

A set of six physically interesting wide gap dielectric materials are investigated. These materials are (1) $\mathrm{Csl}$, (2) sapphire $\left(\mathrm{Al}_{2} \mathrm{O}_{3}\right),(3) \mathrm{CaF}_{2}$, (4) DKDP $\left(\mathrm{KH}_{\times} \mathrm{D}_{(2-x)} \mathrm{PO}_{4}\right)$, (5) fused silica $\left(\mathrm{SiO}_{2}\right)$, and (6) LiF. Each sample was individually irradiated with 355-nm, 532-nm and 1064-nm laser pulses focused in the bulk of the materials. Pulses were of duration $2.7 \mathrm{~ns}, 4.2 \mathrm{~ns}$, and $5.5 \mathrm{~ns}$ respectively. Because the laser damage threshold is both material and wavelength dependent, a wide range of laser fluences were used [12]. For example, the fluences used in DKDP ranged from $16 \mathrm{~J} / \mathrm{cm}^{2}$ for $355-\mathrm{nm}$ irradiation to the order of $100 \mathrm{~J} / \mathrm{cm}^{2}$ for $1064 \mathrm{~nm}$ irradiation. The irradiation fluences were chosen to be approximately double the damage threshold of each material at a particular wavelength. In all cases, the power used was at least an order of magnitude below the self focusing thresholds. The emission spectra and the fits to the Planckian distribution for each of the materials are shown in figures 5.5 to 5.10. An illustration of the error in the temperature fits is shown in figure 5.11. 


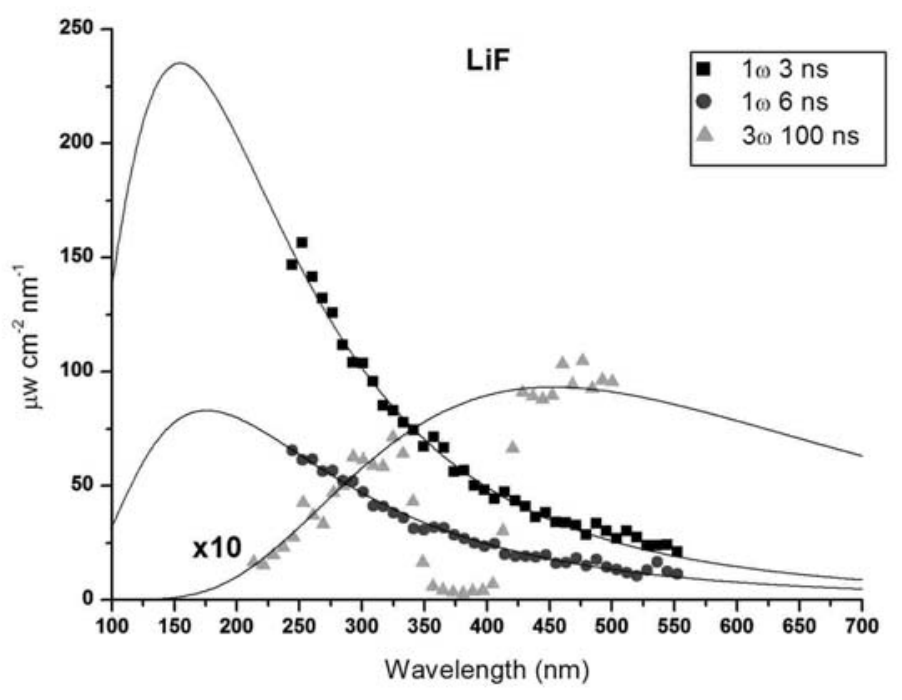

Figure 5.5

Emission spectra accompanying laser-induced breakdown in LiF from 1064-nm pulses $3 \mathrm{~ns}$ and $6 \mathrm{~ns}$ after the peak of the pulse and $100 \mathrm{~ns}$ after the peak of a 355-nm pulse. The solid symbols are the representative experimental data (only every $15^{\text {th }}$ point is shown for clarity). The solid lines are the fits to the Planckian distribution. The 3- $\omega 100-n s$ data and fit have been multiplied by 10 to enhance their visibility.

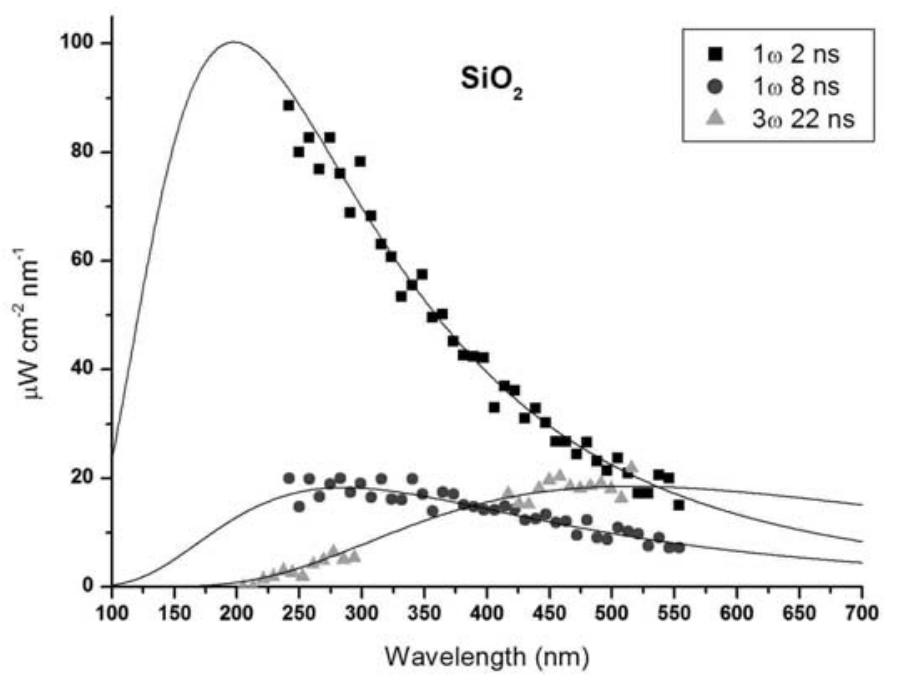

Figure 5.6

Emission spectra accompanying laser-induced breakdown in $\mathrm{SiO}_{2}$ from 1064-nm pulses $2 \mathrm{~ns}$ and $8 \mathrm{~ns}$ after the peak of the pulse and $22 \mathrm{~ns}$ after the peak of a 355-nm pulse. The solid symbols are the representative experimental data (only every $15^{\text {th }}$ point is shown for clarity). The solid lines are the fits to the Planckian distribution. 


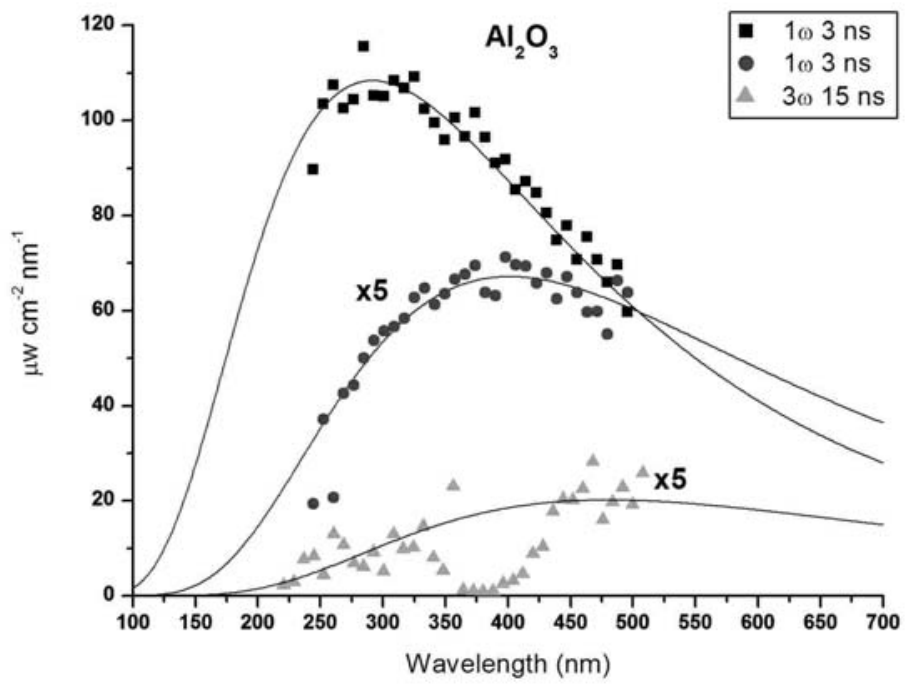

Figure 5.7

Emission spectra accompanying laser-induced breakdown in $\mathrm{Al}_{2} \mathrm{O}_{3}$ from 1064$\mathrm{nm}$ pulses $3 \mathrm{~ns}$ and $30 \mathrm{~ns}$ after the peak of the pulse and $15 \mathrm{~ns}$ after the peak of a $355-\mathrm{nm}$ pulse. The solid symbols are the representative experimental data (only every $15^{\text {th }}$ point is shown for clarity). The solid lines are the fits to the Planckian distribution. The 1- $\omega$ 30-ns and 3- $\omega$ 15-ns data and their fits have been multiplied by a factor of 5 to enhance their visibility. 


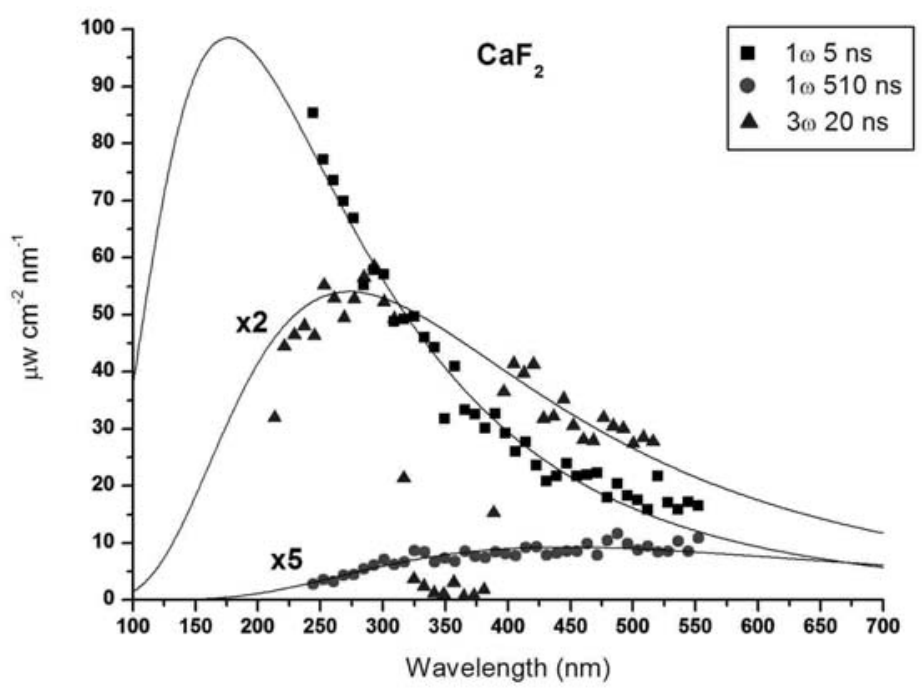

Figure 5.8

Emission spectra accompanying laser-induced breakdown in $\mathrm{CaF}_{2}$ from 1064-nm pulses $5 \mathrm{~ns}$ and $510 \mathrm{~ns}$ after the peak of the pulse and $20 \mathrm{~ns}$ after the peak of a $355-\mathrm{nm}$ pulse. The solid symbols are the representative experimental data (only every $15^{\text {th }}$ point is shown for clarity). The solid lines are the fits to the Planckian distribution. The 1- $\omega 510-n s$ and $3-\omega 20-n s$ data and fits have been multiplied by 5 and 2 , respectively, to enhance their visibility. 


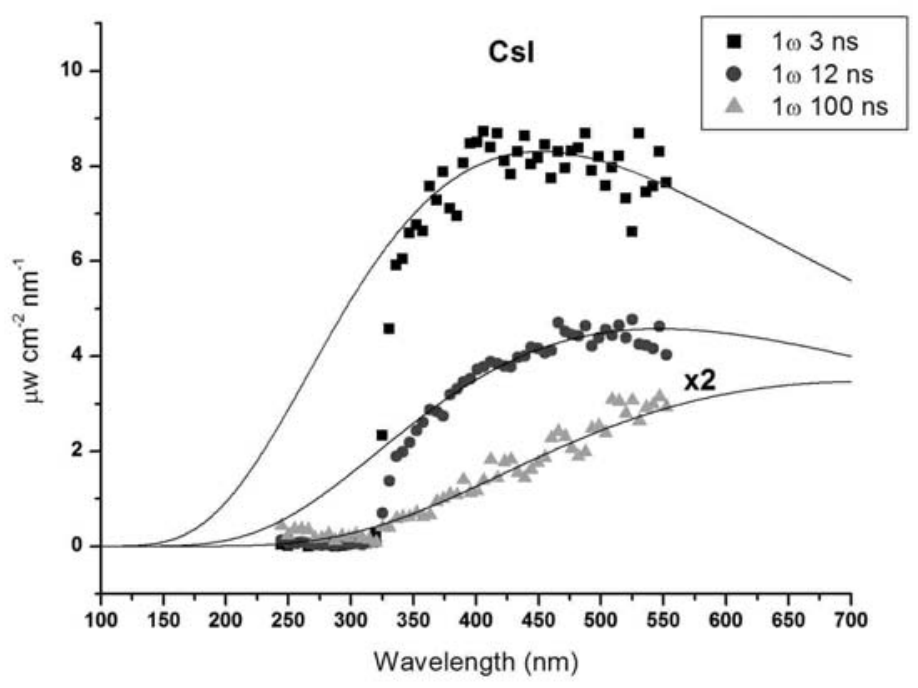

Figure 5.9

Emission spectra accompanying laser-induced breakdown in CsI from 1064-nm pulses $3 \mathrm{~ns}, 12 \mathrm{~ns}$, and $100 \mathrm{~ns}$ after the peak of the pulse. The cut off in the data at $\sim 325 \mathrm{~nm}$ is due to the absorption edge of Csl. The solid symbols are the representative experimental data (only every $15^{\text {th }}$ point is shown for clarity). The solid lines are the fits to the Planckian distribution with the data below $350 \mathrm{~nm}$ ignored during fitting. The 1- $\omega 100-n s$ data and fit have been multiplied by 2 to enhance their visibility 


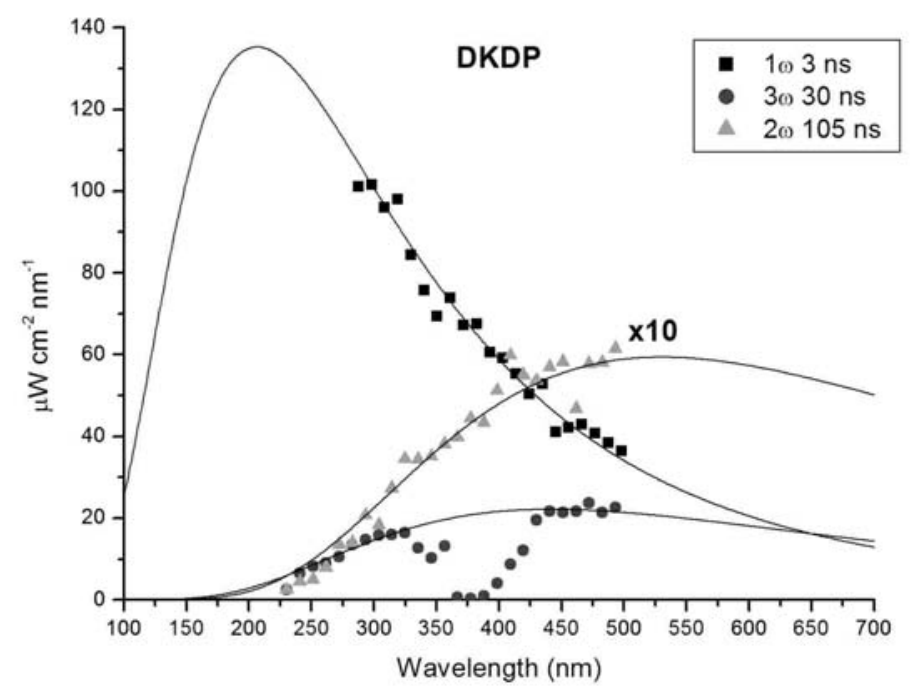

Figure 5.10

Emission spectra accompanying laser-induced breakdown in DKDP from 1064$\mathrm{nm}, 355-\mathrm{nm}$ and 532-nm pulses $3 \mathrm{~ns}, 30 \mathrm{~ns}$, and $105 \mathrm{~ns}$, respectively, after the peak of the pulse. The solid symbols are the representative experimental data (only every $15^{\text {th }}$ point is shown for clarity). The solid lines are the fits to the Planckian distribution. The 2- $\omega 105-n s$ data and fit have been multiplied by 10 to enhance their visibility 


\subsection{Discussion}

\section{a. Error analysis}

The error in the temperature is evaluated by first smoothing a data set with a Stineman function and adding error bars to the smoothed data to encompass the unsmoothed data to $2 \sigma$. Next the original (unsmoothed) data is fit to the Planckian distribution to determine the best fit temperature. This best fit is plotted with the smoothed data along with Planckian distributions in which the temperatures are forced to be above or below the best fit value. The error in the temperature is estimated by the temperature displacement needed to force the Planckian distribution outside the error bars associated with the smoothed data. The results of the described procedure, visible in figure 5.11, indicate that the error is approximately $300 \mathrm{~K}-500 \mathrm{~K}$ for a $4700 \mathrm{~K}$ temperature. 


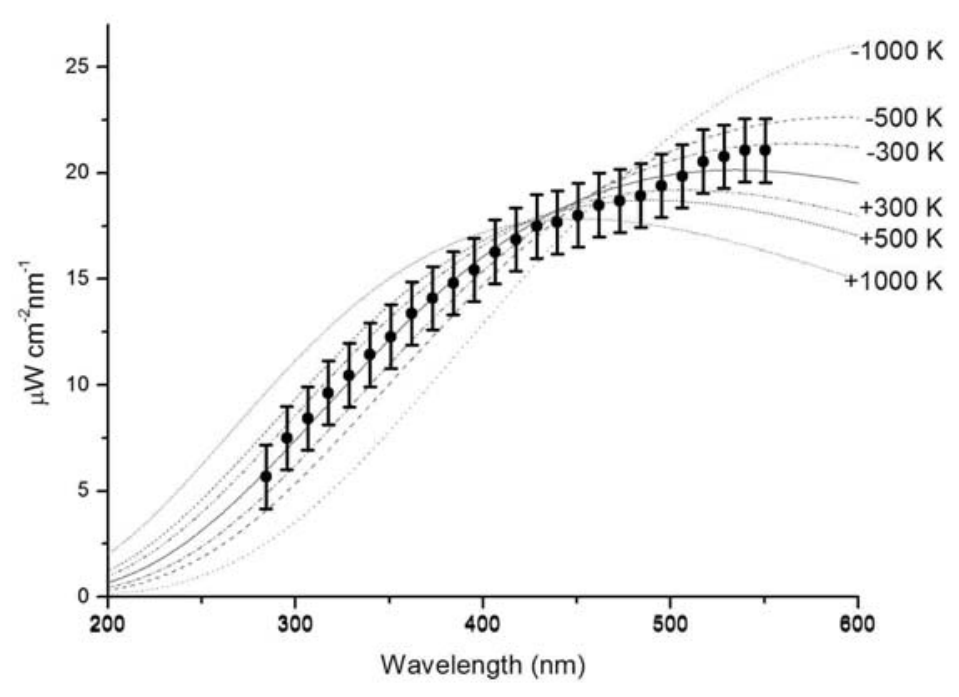

Figure 5.11

The solid dots are a calibrated data set smoothed with a Stineman function. The error bars encompass the unsmoothed data points to the $2 \sigma$ level. The central solid line indicates the best fit Planckian distribution (with a temperature of $\sim 4700$ $\mathrm{K})$. The 3 lines above and below the best fit show the fits where the temperatures are forced to vary from best fit value while the magnitude (effective emissivity) is allowed to float.

From this an error of $\sim 8 \%$ will be estimated for all temperature measurements. It is important to note that only the shape of the curve determines the temperature. This feature is critical as the intensity of the spectra varies drastically with laser fluence, and to some extent, shot to shot with relatively constant fluences. This is consistent with the variation observed in the effective emissivity measurements. 


\section{b. Temperatures}

The plasma temperature as a function of time is insensitive to the wavelength used to initiate breakdown. Figure 5.12 shows temperature vs. time data for $\mathrm{SiO}_{2}$ for 355-nm, 532-nm, and 1064-nm experiments. Within the error assigned to the measurements, the temperatures produced by the various pump wavelengths are indistinguishable. Because of the greater useable range, both temporally and spectrally, the experiments in figures $5.12-5.17$ show temperature vs. time data for all six materials for 1064-nm experiments.

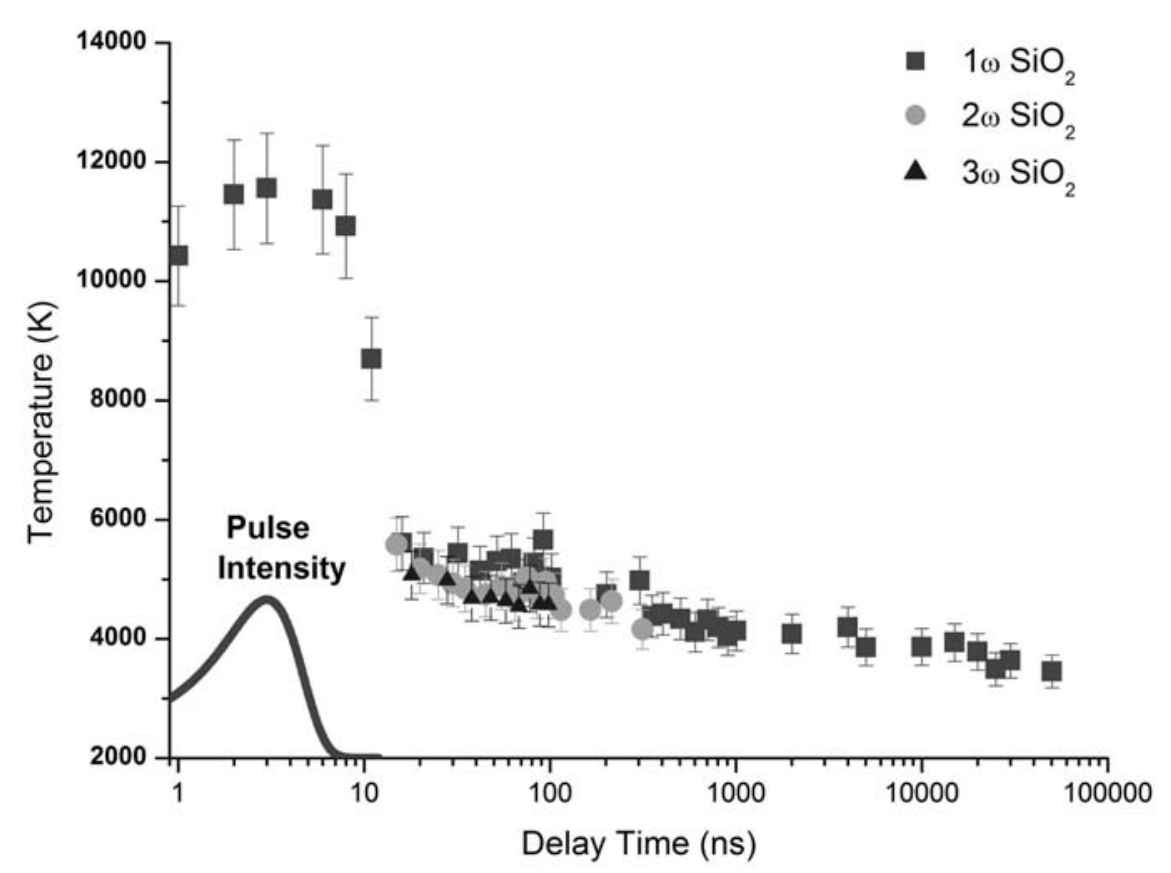

Figure 5.12

Temperature vs. time data taken in $\mathrm{SiO}_{2}$ for $355-\mathrm{nm}, 532-\mathrm{nm}$, and 1064-nm experiments. The temperatures obtained from the various pump wavelengths are indistinguishable within the error of the measurement. A schematic representation of the 1064-nm laser intensity is shown in arbitrary units. 


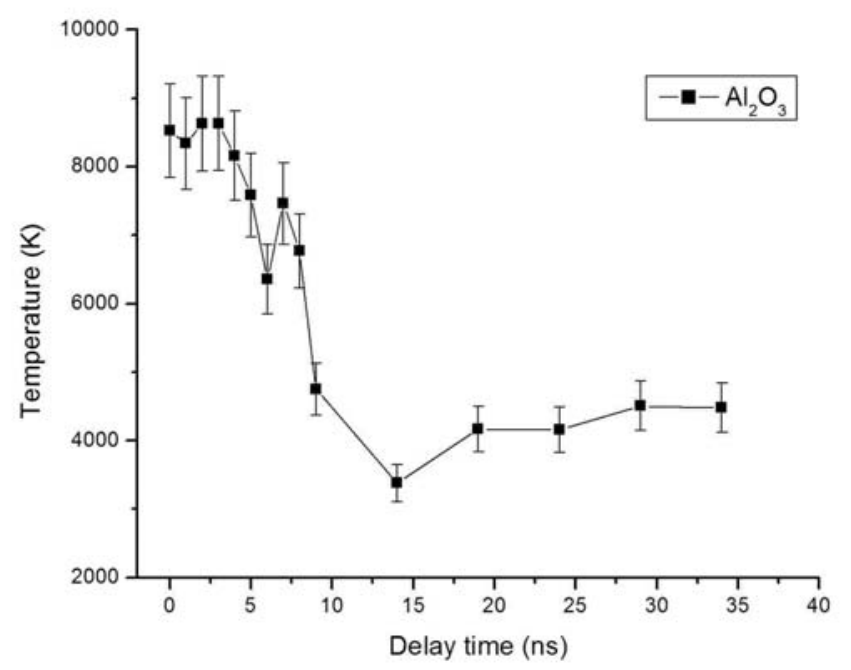

Figure 5.13

Temperature vs. time data for $\mathrm{Al}_{2} \mathrm{O}_{3}$ taken with 1064-nm pump radiation. The temperature is seen to fall rapidly after the termination of the laser pulse and then decreases at a slower rate.

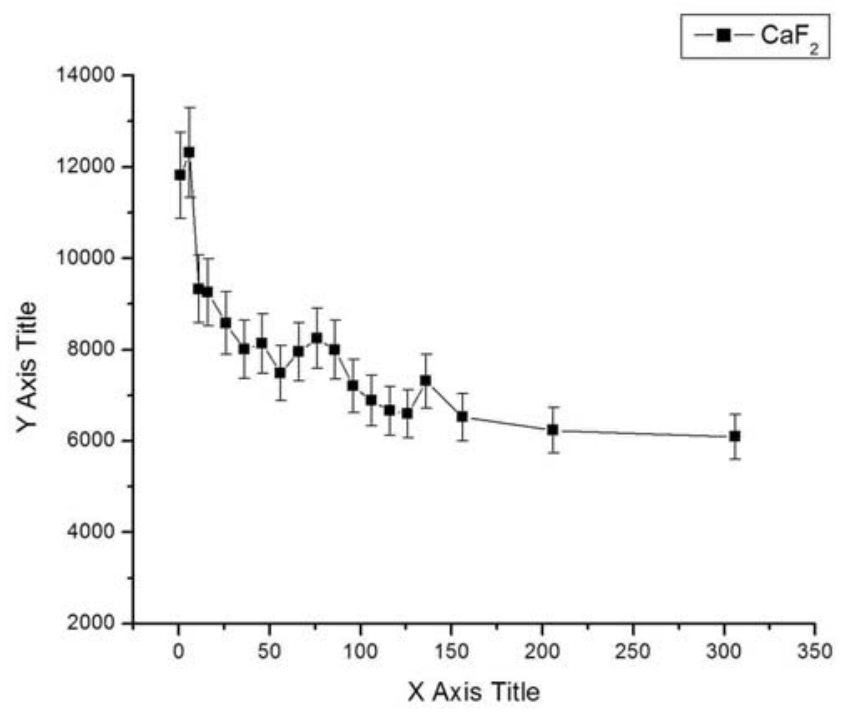

Figure 5.14

Temperature vs. time data for $\mathrm{CaF}_{2}$ taken with 1064-nm pump radiation. The temperature is seen to fall rapidly after the termination of the laser pulse and then decreases at a slower rate. 


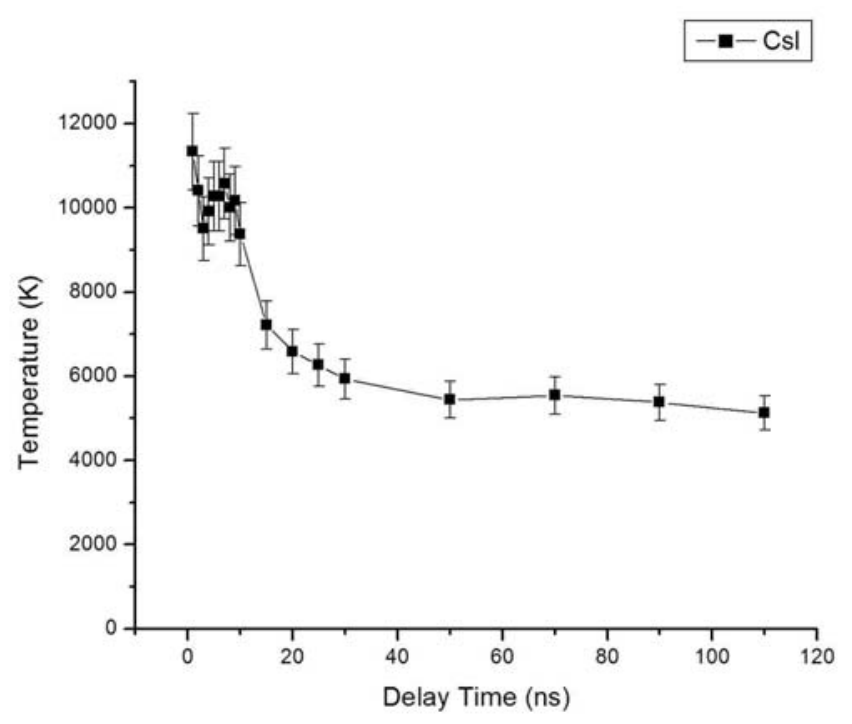

Figure 5.15

Temperature vs. time data for CsI taken with 1064-nm pump radiation. The temperature is seen to fall rapidly after the termination of the laser pulse and then decreases at a slower rate.

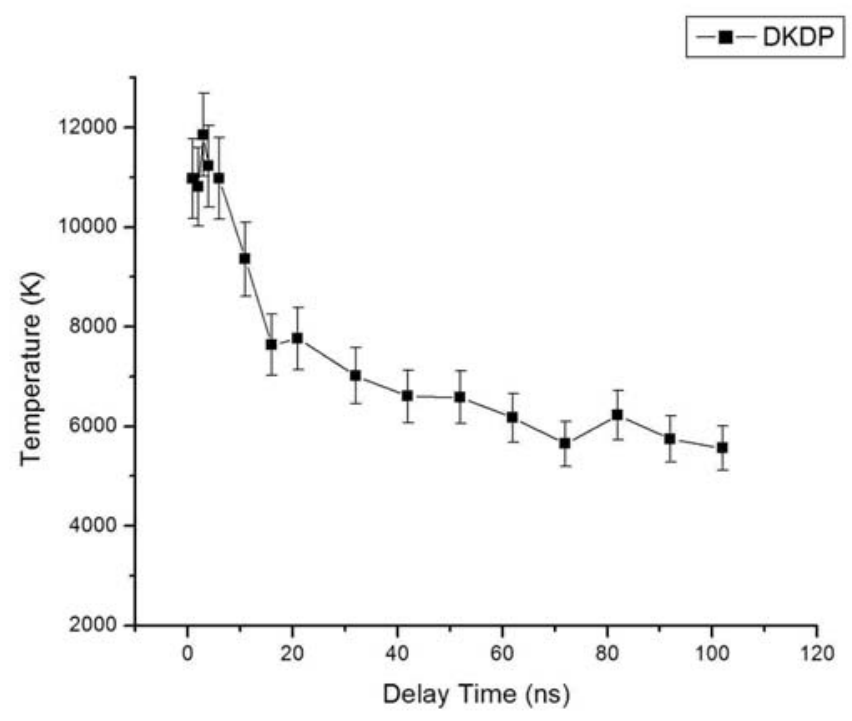

Figure 5.16

Temperature vs. time data for DKDP taken with 1064-nm pump radiation. The temperature is seen to fall rapidly after the termination of the laser pulse and then decreases at a slower rate. 


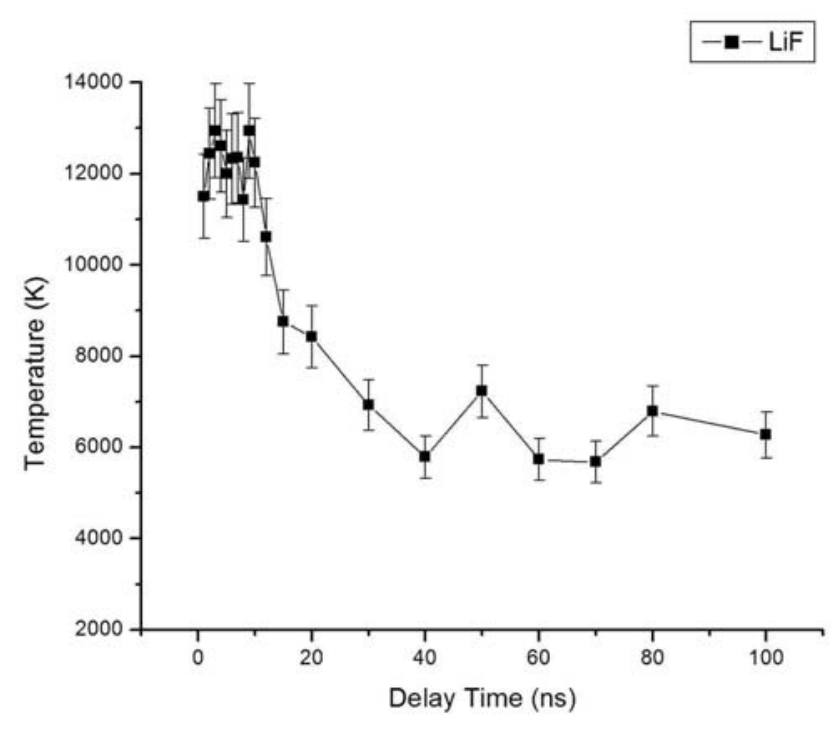

Figure 5.17

Temperature vs. time data for LiF taken with 1064-nm pump radiation. The temperature is seen to fall rapidly after the termination of the laser pulse and then decreases at a slower rate.

In order to compare the various materials, the peak temperature observed in each is plotted vs. the band gap of the material in figure 5.18.

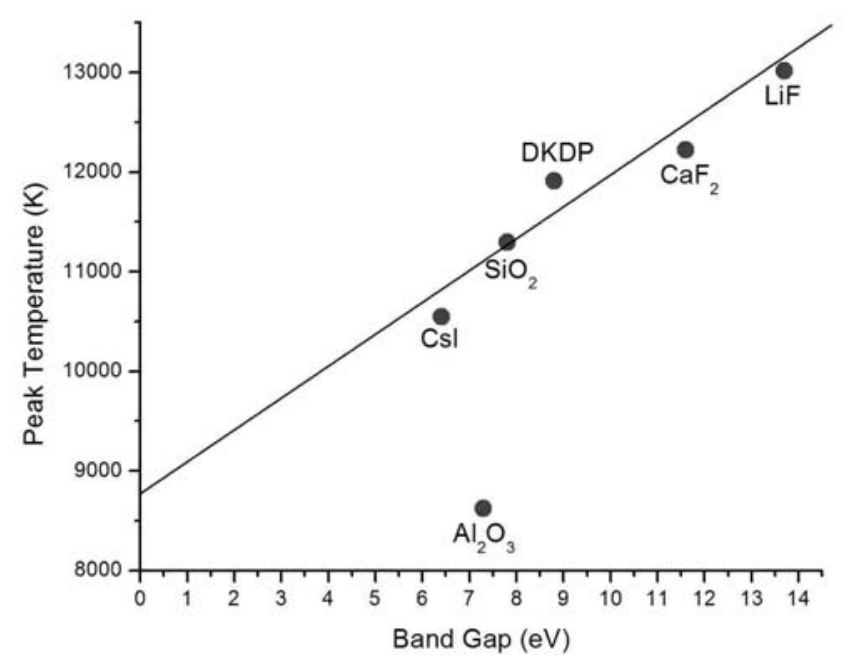

Figure 5.18

Peak temperature of the materials is plotted vs. material band gap. The straight line is a guide to the eye which suggests a linear relationship between band gap and peak temperature. The reduced temperature observed in sapphire is consistent with the large thermal conductivity of the material. 
Overall, the lowest temperatures were observed in sapphire. This effect can be attributed to its high thermal conductivity. The maximum temperatures were observed in LiF which has the largest band gap $E_{g}$ among the materials under investigation. The relation of the peak temperature to band gap can be attributed to the larger energies needed to liberate an electron in the wider gap materials.

\begin{tabular}{|c|c|c|c|c|c|}
\hline Sample & $\mathrm{T}_{3}(\mathrm{~K})$ & $\begin{array}{c}\mathrm{T}_{20} \\
(\mathrm{~K})\end{array}$ & $\begin{array}{c}\mathrm{T}_{100} \\
(\mathrm{~K})\end{array}$ & $\begin{array}{c}\mathrm{E}_{\mathrm{g}} \\
(\mathrm{eV})\end{array}$ & $\sigma\left(\frac{\mathrm{W}}{m K}\right)$ \\
\hline $\mathrm{LiF}$ & 12400 & 8400 & 6300 & $13.7[13]$ & $4.01[14]$ \\
\hline $\mathrm{CaF} 2$ & 12200 & 9300 & 7200 & $11.6[15]$ & $9.71[14]$ \\
\hline $\mathrm{DKDP}$ & 11800 & 7800 & 5600 & $8.4[16]$ & $1.9[17]$ \\
\hline $\mathrm{SiO}_{2}$ & 11500 & 5400 & 4700 & $7.8[18]$ & $1.38[14]$ \\
\hline $\mathrm{Csl}$ & 10400 & 6600 & 5200 & $6.4[19]$ & $1.1[14]$ \\
\hline $\mathrm{Al}_{2} \mathrm{O}_{3}$ & 8300 & 4200 & $*$ & $7.3[18]$ & $27.21[14]$ \\
\hline
\end{tabular}

Table 5.1

$\mathrm{T}_{3}, \mathrm{~T}_{20}$, and $\mathrm{T}_{200}$ are the temperatures of each material at delays of $3 \mathrm{~ns}, 20 \mathrm{~ns}$, and $100 \mathrm{~ns}$ respectively. The room temperature values of band gap $\left(E_{g}\right)$ and thermal conductivity $(\sigma)$ for each material are also listed. *Signal was below the experimental detection limit. 


\section{$5.4 \quad$ References}

[1] Gorbunov, A.V., et al., Fiz Tverd Tela 36, 1429-36 (1994).

[2] Carr, C.W., et al., SPIE, 2001. 4679: p. 360.

[3] Claeyssens, F., et al., J. Appl. Phys. (USA) 91, 6162-6172 (2002).

[4] Baghdassarian, O., et al., Phys Rev Lett 86, 4934-4937 (2001).

[5] Heszler, P., et al., J. Appl. Phys. (USA) 89, 3967-3970 (2001).

[6] Shen, Z.H., et al., Microw Opt Techn Let 25, 231-233 (2000).

[7] Geohegan, D.B., Appl Phys Lett 62, 1463-1465 (1993).

[8] Berthe, L., et al., J. Appl. Phys. (USA) 82, 2826-2832 (1997).

[9] Kawaguchi, Y., et al., Appl Surf Sci 197, 268-272 (2002).

[10] Williamson, J.M., et al., J. Appl. Phys. (USA) 93, 1893-1898 (2003).

[11] Bandis, et al., Appl Surf Sci 197, 100-106 (2002).

[12] Carr, C.W., et al., Physical Review Letters (2003).

[13] Fox, M., Optical Properties of Solids (Oxford University Press, 2001).

[14] Crystran, Crystan Technical Handbook of Materials. 2003: Dorset.

[15] Barth, J., et al., Physical Review B Condensed Matter 41, 3291-4 (1990).

[16] Ogorodnikov, I.N., et al., Optika i Spektroskopiya 91, 243-51 (2001).

[17] Suemune, Y., Journal of the Physical Society of Japan 22, 735-43 (1967).

[18] Desalvo, R., et al., IEEE J. Quantum Electron. (USA) 32, 1324-1333 (1996).

[19] Radousky, H.B., et al., Physical Review B Condensed Matter 31, 1457-62 (1985). 


\section{Chapter 6}

\section{Discussion and Conclusions Concerning Laser-Induced Damage in Wide Band Gap Optical Materials}

\subsection{Introduction}

In this chapter both the physical meaning of the experiments and the requirements for the models discussed in the preceding chapters will be discussed in terms of the three phases of laser-induced damage. The damage initiation phase is discussed by first reviewing models from the literature and their predicted wavelength dependent damage thresholds. The behavior predicted by these models is then compared to that observed in Chapters 2 and 3 . As none of the models from the literature fully fit the experimental observations, the necessity of a new hybrid model is reviewed and the requirements for the model to be viable are expanded upon. 
These requirements include the presence of a defect population which is capable of supplying defect states in the band gap under some conditions, but not all. Experiments which show defect clusters present in KDP which exhibit behavior consistent with the laser condition effect are discussed along with the response of these defect clusters to low power radiation. The behavior of the required defects is compared to the properties of hydrogen displacement defects, recently calculated for KDP by Liu et al. Hydrogen displacement defects are shown to produce states in the gap and to be capable of transition from stable to meta-stable configurations [1].

Once a plausible explanation for damage initiation is established, the second and third phases of laser-induced damage are considered. Insight is gained into both energy absorption and dissipation by further analysis of the plasma temperatures measured in Chapters 4 and 5 . Using the StefanBoltzmann law in conjunction with the equation of state for fused silica, the temperatures, pressures, and energy densities obtained during the energy absorption phase are considered. The temporal evolution of the radiation profile is then used to determine the relative roles of the various energy dissipation mechanisms. 


\subsection{Discussion}

The objective of this dissertation has been twofold: to gain an understanding of the mechanisms responsible for laser-induced damage occurring at lower than extrinsic breakdown levels, and to understand the processes which take place during each of the three phases of laser-induced damage. The wavelength dependence of damage initiation is now used to evaluate the proposed models for damage initiation. The models describing the first phase of laser-induced damage include impact ionization, absorbing nanoparticles, and multi-photon ionization. The wavelength dependence predicted by each of these models can be seen in figures 6.1 and 6.2. By comparing the behavior predicted by the various models to that which is observed, the mechanisms involved in damage initiation are elucidated.

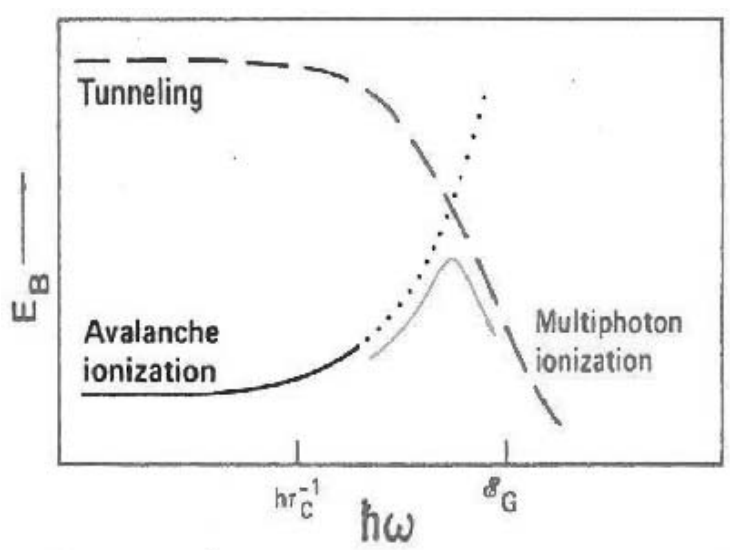

Figure 6.1

From Bloembergen and Jones et al.[2, 3] The wavelength dependence of the damage threshold predicted by the impact ionization and multi-photon models.

In figure 6.1 the wavelength dependence of the impact ionization and multi-photon ionization models are shown. In the impact ionization model the damage threshold is expected to remain constant until the frequency of the 
radiation becomes comparable with the inverse of the electron-lattice collision time $\left(\tau_{\mathrm{e}}{ }^{-1}\right)$. Once the frequency is on the same order or larger than $\left.\tau_{\mathrm{el}}\right|^{-1}$, electrons will tend to lose as much energy to the oscillating field as they gain, increasing the field intensity necessary for breakdown as the laser frequency becomes large.

The breakdown threshold due to multi-photon ionization has a frequency response nearly opposite to that of impact ionization; where photon energy increases, the damage threshold will decrease. The reduction in damage threshold will be abrupt and occur as photon energies reach a smaller fraction of the band gap. e.g. when $h v$ changes from $\left(\varepsilon_{g} / n\right)-\Delta \varepsilon$ to $\left(\varepsilon_{g} / n\right)+\Delta \varepsilon$, where $\left(\varepsilon_{g} / n\right)$ is any fraction of the band gap. The sharp drop in damage threshold results from fewer photons needing to be absorbed simultaneously to liberate an electron. Both the impact ionization and multi-photon mechanisms are electronic in nature, and therefore depend on generating enough free electrons to form a plasma of critical electron density.

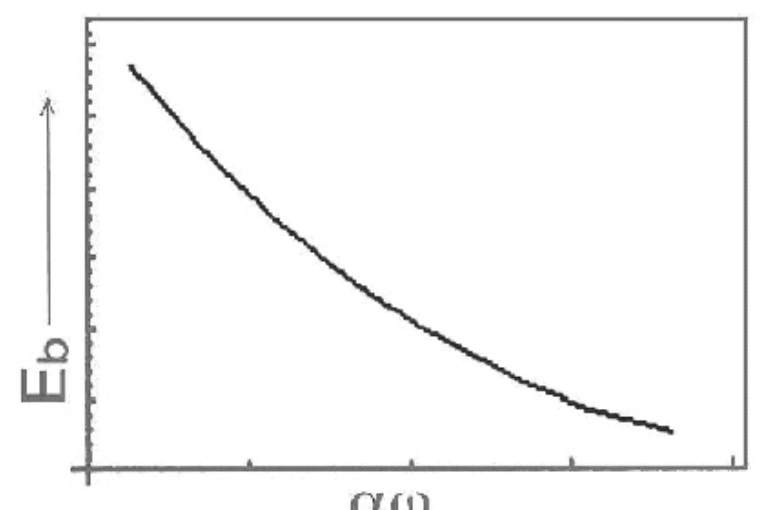

$\alpha(\omega)$

Figure 6.2

From Feit et al.[4] The wavelength dependence of laser-induced breakdown threshold predicted by the gray particle model, assuming a uniform size distribution of inclusions. 
The gray particle model requires inclusions of foreign material to be imbedded in the normally transparent host. It must be emphasized that the wavelength dependence shown in figure 6.2 is for a uniform particle size distribution only. The actual response from imbedded particles could be nearly anything, but, there is no reasonable size distribution of particles which could account for sharp steps in the damage threshold [5]. In this model, the particles are expected to absorb some fraction of whatever light is incident on them. As the inclusions heat they will eventually melt the surrounding lattice, turning it opaque. This opaque material can then rapidly absorb the large amounts of energy necessary to produce the effects associated with laser-induced damage.

Now that the potential damage initiation mechanisms have been outlined, key issues in interpretation of the experimental results must be considered. Throughout the literature many different values have been quoted for the bulk laser-induced damage threshold of KDP and its deuterated analog DKDP [6-8]. The spread in the data is not surprising considering that laser-induced damage thresholds in optical materials depend on the duration of the laser pulse, focal spot geometry, variations between samples, previous exposure of the sample to laser radiation, and experimental technique [9-14] (the control of these parameters is discussed in Chapter 4). These issues, when not addressed, can lead to a variation in experimental results of nearly two orders of magnitude. Consequently, this has made impossible a quantitative study of the wavelength dependent damage threshold by compiling work from many authors $[6,8]$. 
This issue is addressed in this work by making the comprehensive multiwavelength damage threshold measurement in DKDP discussed in Chapters 2 and 3. Because of the nature of laser-induced damage for nanosecond pulses, the damage threshold is most accurately described in probabilistic terms. The lowest fluence at which a sample will damage $100 \%$ of the time is in fact the intrinsic damage threshold of the material. In materials which are less than ultrapure, this value is difficult to determine, as well as fairly useless for describing the global behavior of a sample because the damage threshold can vary from site to site by an order of magnitude for a small enough beam. The fluence at which a site will damage $50 \%$ of the time, however, can be easily defined and gives information relevant to realistic performance about an individual crystal. For this reason, the samples susceptibility to damage as a function of wavelength is determined by producing a series of damage probability vs. fluence 's-curves', at each wavelength (as discussed in Chapter 2). When the 50\% damage threshold is plotted vs. photon energy (figure 6.3), a behavior is observed which does not fit well to any of the above models. It should be noted that this is in contrast to the treatment in Chapter 3 where the same data was plotted as a function of wavelength. 


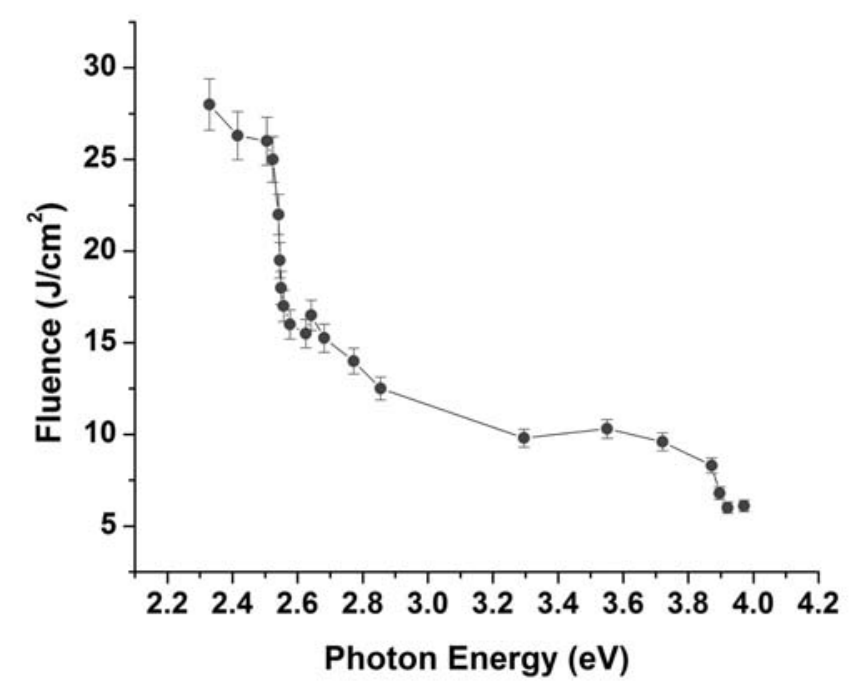

Figure 6.3

The $50 \%$ damage threshold as a function of photon energy.

The wavelength dependence of the damage threshold appears to be qualitatively closest to the multi-photon mechanism. However, the damage morphology and the observed values of the damage threshold are completely inconsistent with a pure multi-photon model. The gray particle model does predict the correct damage morphology, and accounts for the fluences at which the sample is observed to damage, but it does not account for the sharp steps in the damage threshold. The impact ionization model accounts for none of the observed behavior. Although it is possible that impact ionization is a participant once another mechanism has initiated damage, it must be discounted entirely as the primary damage initiation mechanism $[11,15]$.

Using these observations the hybrid model proposed in Chapter 3, Defect Assisted Multi-Photon (DAMP) ionization, is now discussed in greater detail. In the proposed model, impurities or lattice defects incorporated into the host material produce states within the gap. These states are analogous to stepping 
stones across a stream for electrons transitioning from the valence band to the conduction band. Because the defects are localized, potential even concentrated in clusters, the expected damage morphology from this mechanism matches that which is observed. This mechanism will also account for the sharp steps observed in the damage threshold. The states within the gap only facilitate transfer of electrons from valence to conduction band, but do not alter the energy necessary for the transition. For this reason, when the order of the multi-photon process (the number of photons needed to provide sufficient energy for an electron to be freed from the valence to the conduction band) changes, a sharp change in the damage threshold is expected. Because the absorption process is made more likely (photons do not need to be absorbed simultaneously) the overall damage threshold is expected to be less that the extrinsic damage threshold, which is also the case.

Figure 6.4 illustrates how this mechanism can account for the steps in the damage threshold. On the right side of the figure the vertical arrows represent electron gaining energy by absorbing photons in order to transition from the valence band to the condition band (represented by the horizontal lines at the bottom and top of the figure, respectively). The length of the arrows corresponds to the energy of the photon absorbed. The figure illustrates how $42.4 \mathrm{eV}$ photons are needed to cross the band gap, but at $2.5 \mathrm{eV}$ only 3 photons are needed. This is again shown for the second change in order ( 3 photons to 2 photons) between $3 \mathrm{eV}$ and $3.8 \mathrm{eV}$. 


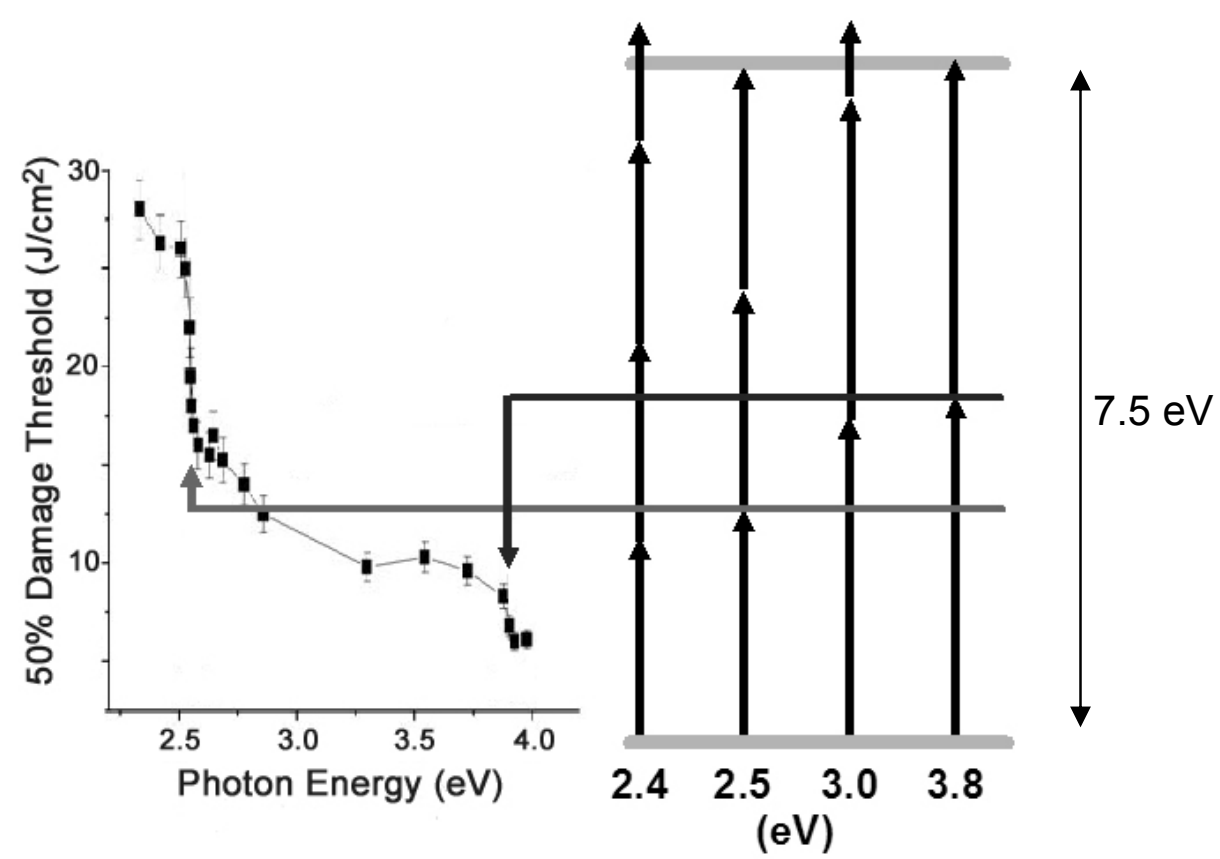

Figure 6.4

A schematic representation of how multi-photon absorption can lead to the steps observed in the damage threshold in figure 3.3. A band gap value of $7.5 \mathrm{eV}$ is used for simplicity.

The phenomenological model proposed here assumes the existence of intra-band defects, and there is a reasonable expectation that these types of defects exist in DKDP. An example of a class of defects which have the properties needed are hydrogen vacancies, interstitial hydrogen atoms or Frenkel pairs (an interstitial hydrogen near a vacancy). Such defects have recently been considered theoretically by Liu et al., and can easily be included during growth or can result from impurity ion incorporation (see figure 6.5) [1]. 

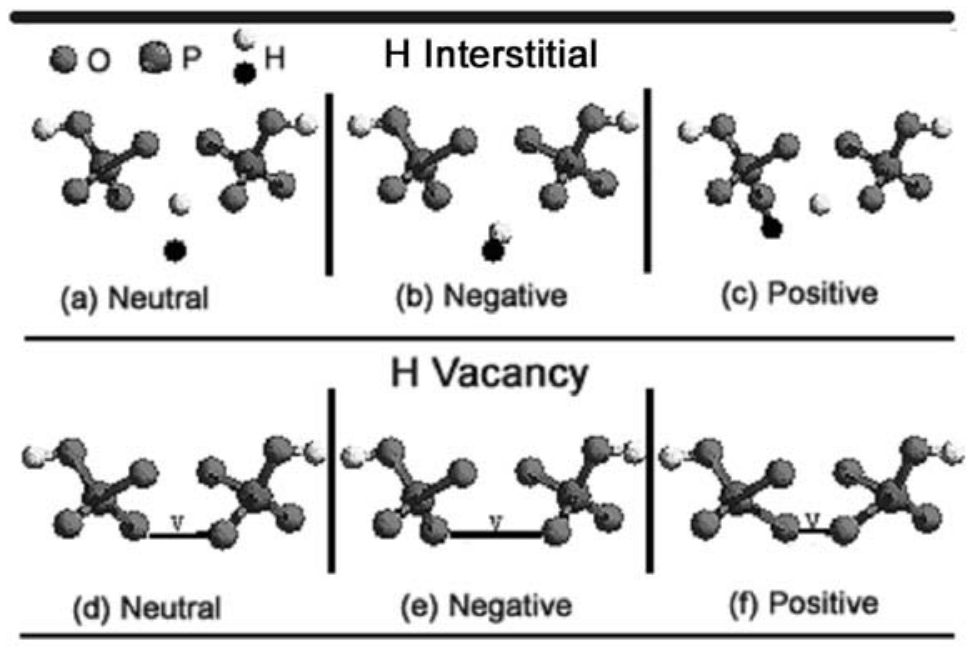

Figure 6.5

From [1]. Six types of defects calculated to be present in KDP. In DKDP, the Hydrogen would be replaced with deuterium. "Relaxed atomic configurations and reactions for interstitial $\mathrm{H}$ for the neutral (a), negative (b) and positive (c) charged states. Also we show the relaxed atomic configurations for the $\mathrm{H}$ vacancy in the neutral (d), negative (e) and positive (f) charged states." -Liu et al.

The band structure of these hydrogen defects has been calculated, and as can be seen in figure 6.6, produce the states in the gap required by the proposed defect assisted multi-photon model [1]. 


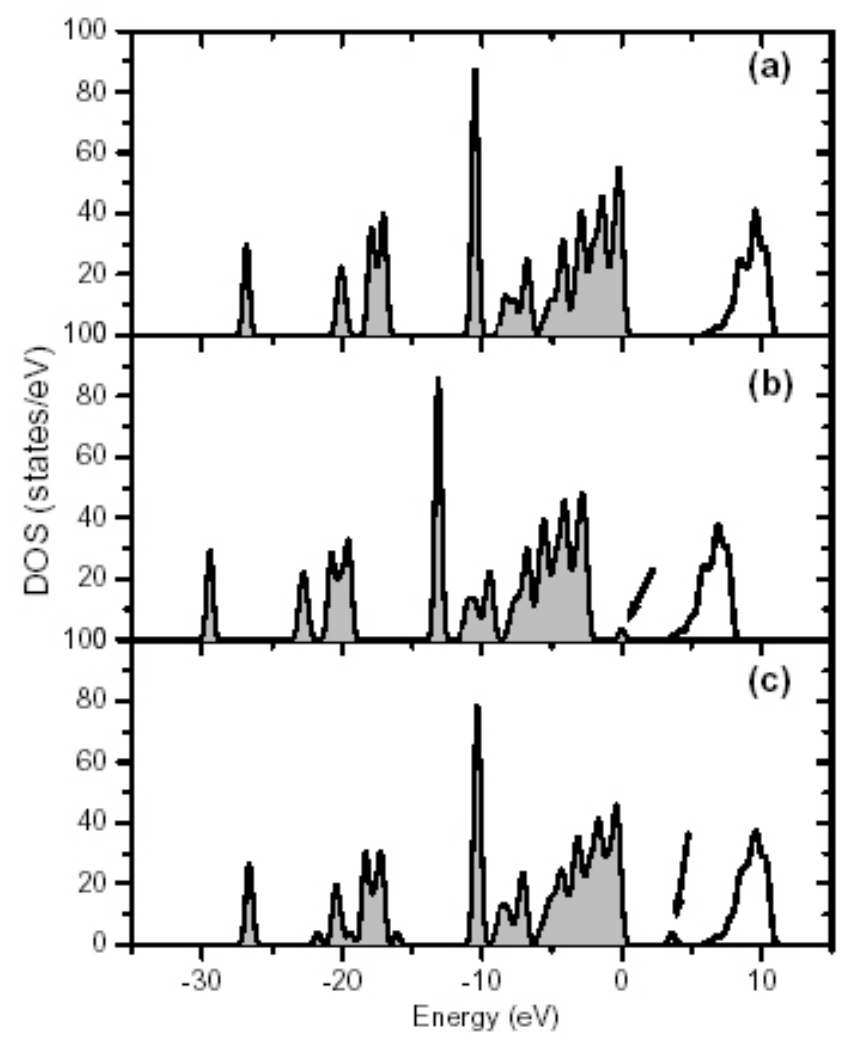

Figure 6.6

From [1]. The density of states calculated by Liu et al. "...pure KDP (a), KDP with an interstitial $\mathrm{H}$ in the neutral state (b), and KDP with a $\mathrm{H}$ vacancy in the positive charged state (c)".

Finally, the density of defects implied by this approach is considered. The overall damage thresholds are reduced because the effective cross section for a multi-step process is larger than that of a single step. For example, the cross section of a three photon process is on the order of $10^{-81} \mathrm{~cm}^{6} \mathrm{~s}^{2}$ while the cross section for a combination of two photon absorption followed by excited state absorption by a single photon is estimated to have an upper bound of $10^{-61}$ $\mathrm{cm}^{6} \mathrm{~s}^{2}$. Using this modified cross section in equation 3.2 with the observed damage threshold, a lower bound to the necessary defect density is estimated to be on the order of $10^{7} \mathrm{~cm}^{-3}$. 
Demos et al. has shown using in-situ florescence microscopy that localized defect concentrations of this magnitude are reasonable in DKDP crystals, as demonstrated by the observation of fluorescing centers in the closely related crystal KDP which had a concentration on the order of $10^{7} \mathrm{~cm}^{-3}$ [16]. An example of data obtained with the in-situ microscopic technique can be seen in figures $6.7 \mathrm{a} \& \mathrm{~b}$.
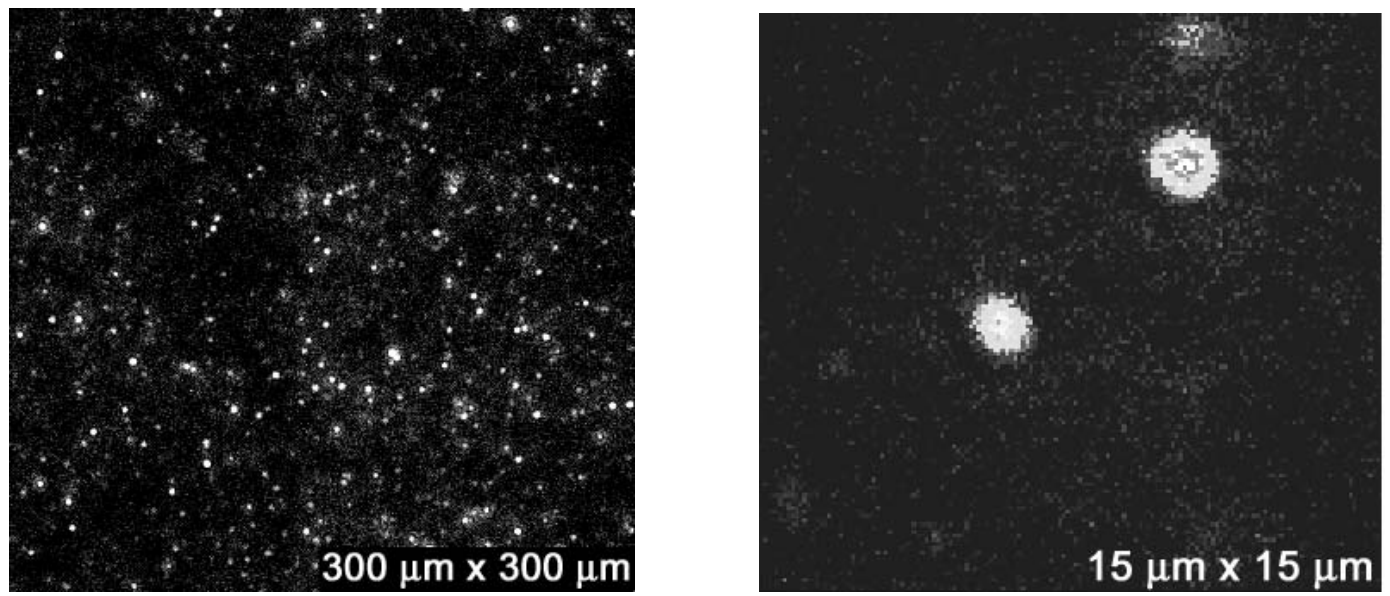

\section{Figure $6.7 \mathrm{a} \& \mathrm{~b}$}

a) Fluorescing centers observed in KDP. b) High resolution image of the fluorescing centers from [16].

If the fluorescing centers are assumed to be clusters of individual defects, the fluorescing center density is a lower bound to the defect density $[16,17]$. The fluorescing centers have been shown to become inactive (no longer fluorescing) under sub damage threshold pulsed irradiation by taking images before and after exposure to 355-nm 3-ns pulses [16]. Although the net number of fluorescing centers is always seen to decrease, the difference image shows new individual centers appearing (see figure 6.8). This behavior is consistent with the hydrogen defect calculations of Liu et al. in that the hydrogen defects can be in one of 
several metastable configurations, some that supplies states within the band gap (allowing florescence) and some that does not.
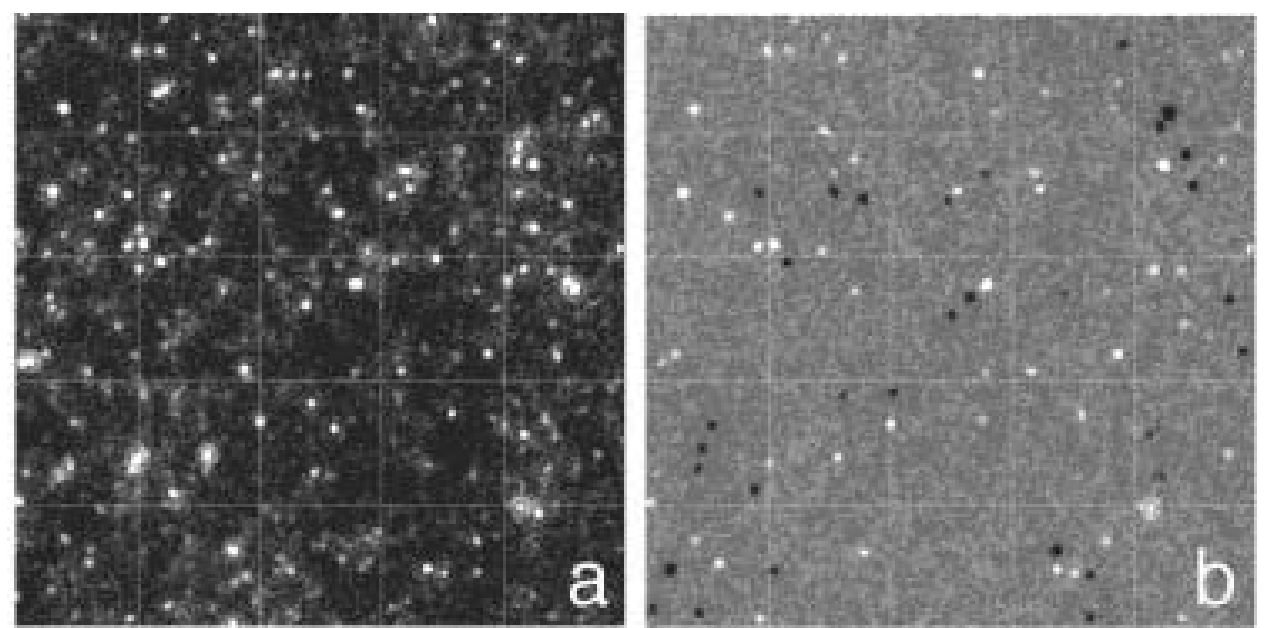

Figure 6.8

a) The original image of fluorescing centers before exposure to 355-nm 3-ns pulsed radiation. b) A difference image showing (white dots) clusters which have disappeared and (black dots) new clusters which have appeared after illumination with pulsed radiation.

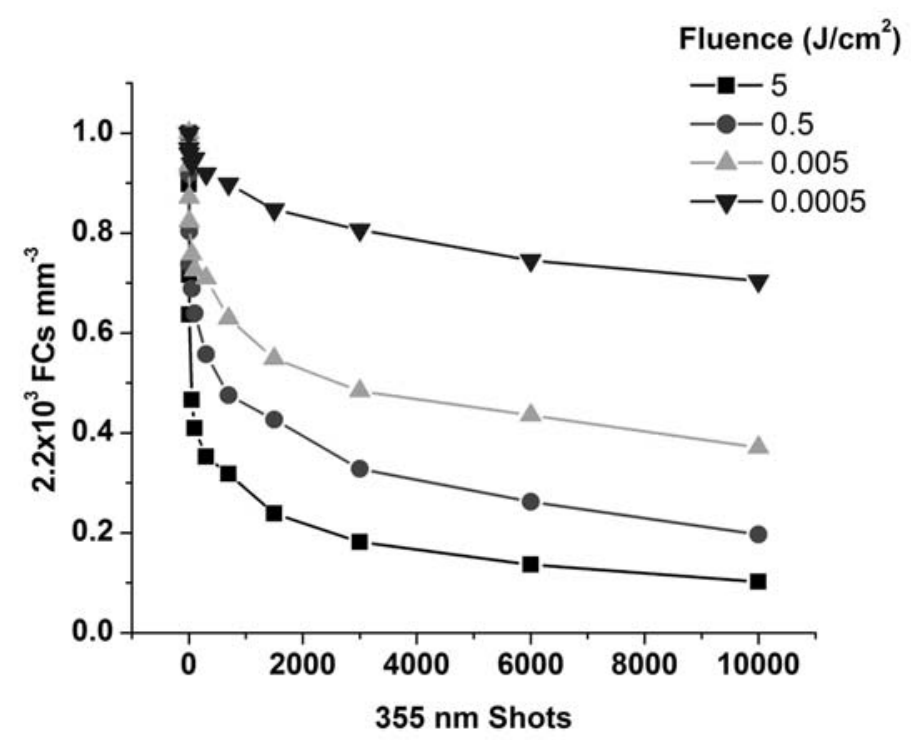

Figure 6.9

The density of fluorescing centers after illumination with between 0 and $10^{4} 355$ $\mathrm{nm}$ 3-ns pulses for four orders of magnitude in fluence.

By studying the defect reactions in more detail, additional information about the dynamics of the clusters' appearance and disappearance can be 
gained. Figure 6.9 shows the density of clusters as a function of the number of illumination pulses for 4 orders of magnitude of fluence. To gain insight into the nature of the fluorescing centers and establish their relevance to laser damage, a conceptual model describing this behavior is needed. There are several observations which can be used to formulate such a model.

1) Both the fluorescing centers (FCs) and the background are rendered inactive by exposure to pulsed radiation.

2) Although new FCs may appear, there is always a net reduction in their number from exposure to pulsed radiation. Likewise, the intensity of the background is always reduced.

3) Some individual FCs remain active irregardless of exposure dose.

From these observations several assumptions are made.

1) The fluorescing centers are clusters formed of individual defects (after Demos et al.)

2) The background florescence is produced by individual defects

3) Clusters may be formed from and dispersed into the background at rates dependent on the populations of clusters and individual defects.

4) Some clusters are "tougher" (more resistant to being rendered inactive by pulsed radiation) than others.

Using these observations and assumptions, we consider a conceptual (and quite speculative) model which has active defects contained within one of two populations. The defects can either be incorporated into a defect cluster (which manifests itself as a fluorescing center), or they may be solitary, and add to the 
background florescence of the crystal. The observed data can then be explained by pulsed radiation prompting the transition of defects from one population to the other (but preferentially to breaking up the clusters and adding to the background). In addition, individual defects can be rendered inactive. A schematic representation of this process is shown in figure 6.10.

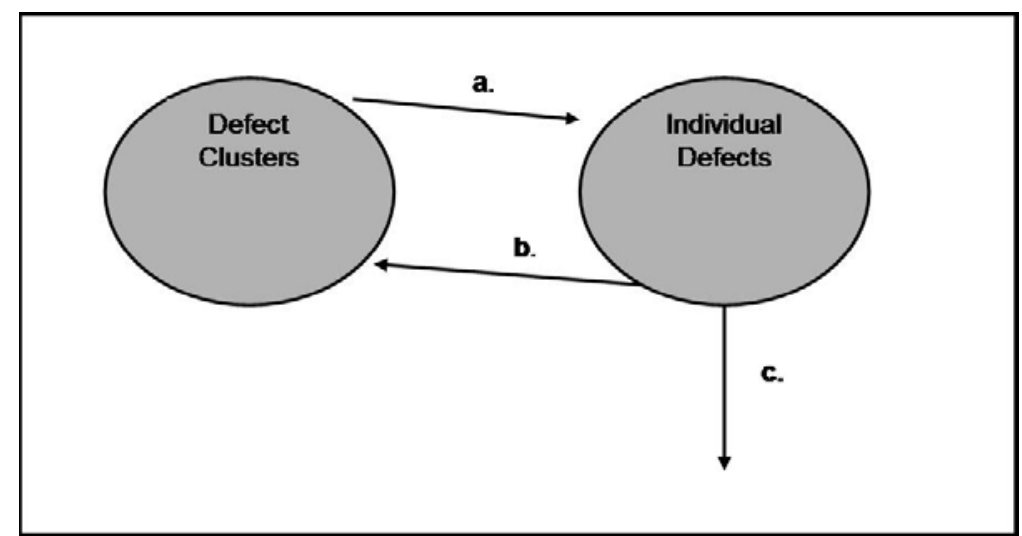

Figure 6.10

A schematic representation of the processes involved in the proposed model. Vectors a. b. and c. represent the rates at which defects are dispersed into the background by removed clusters, incorporated into clusters from the background, and rendered inactive, respectively.

This situation is simulated by first arbitrarily dividing the fluorescing centers into ten distinct populations. The effect of the high energy pulse is simulated by reducing the net number of FCs in each population by

$$
A_{n+1}=A_{n}\left(1-I \cdot \alpha_{A}-I^{2} \cdot \beta_{A}\right)
$$

Where $A_{n+1}$ and $A_{n}$ are the number of FCs in population $A$ after $n+1$ and $n$ "laser pulses". I, $\alpha_{\mathrm{A}}$, and $\beta_{\mathrm{A}}$ are the laser pulse fluence and two parameters reflecting the "toughness" of the centers in population A. For simplicity, the constants for each population were constrained by

$$
\begin{aligned}
& C_{1} \alpha_{X}=\beta_{X} \\
& \alpha_{B}=C_{2} \alpha_{A}, \alpha_{C}=C_{2} \alpha_{D} \text { etc. }
\end{aligned}
$$


where $C_{1}$ and $C_{2}$ are adjustable parameters. These constraints reduce the number of free parameters to $3 ; \alpha_{A}, C_{1}$, and $C_{2}$ Figure 6.11 is produced by performing $10^{4}$ iterations using equation 3.4 to calculate the new FC population after each for several fluences.

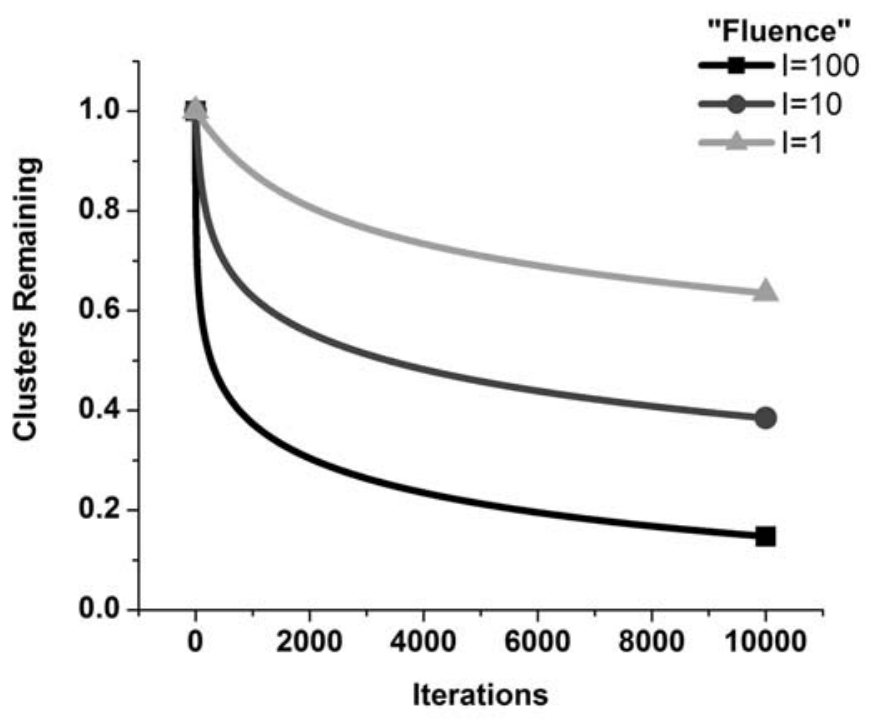

Figure 6.11

The populations of FCs predicted by the model outlined in the text under three different simulated fluences. The qualitative match to the experimental data is exact.

The fit to the model to the show that the rate at which the centers are removed depends on the square of fluence (when exposed to $355-\mathrm{nm}, \sim 3.5 \mathrm{eV}$ radiation). This is consistent with the energy that would be needed to stimulate defects in the range of interest. More specifically, the states produced by the defects must be located from between $\sim 3.5 \mathrm{eV}$ to $7.0 \mathrm{eV}$ from the bottom of the band gap (valance band).

Closer examination of the centers shows that under low power radiation centers are both created and destroyed. This behavior is similar to what would 
be expected by a class of defects which have been calculated to be present in KDP crystals. Hydrogen displacement type defects have been shown to be capable of transitioning between two configurations one of which produces states in the gap, while the other does not. The preferred transition is from the configuration which does produce states in the gap to the configuration which does not. This is consistent with the condition effect. Taken together, it is plausible that these defects are responsible for both the conditioning effect and the reduced damage threshold.

Next, the energy absorption and dissipation phases are considered. The time gated spectroscopic measurements discussed in Chapters 4 and 5 gave insight into the dynamics of the laser-induced damage process. The blackbody emission which accompanies the breakdown process is now considered in greater detail. First, it must be reemphasized that the emission is not, in general, required to be blackbody in nature. In fact, for femtosecond damage initiation the emission is observed not to be Planckian (see figure 6.12). 


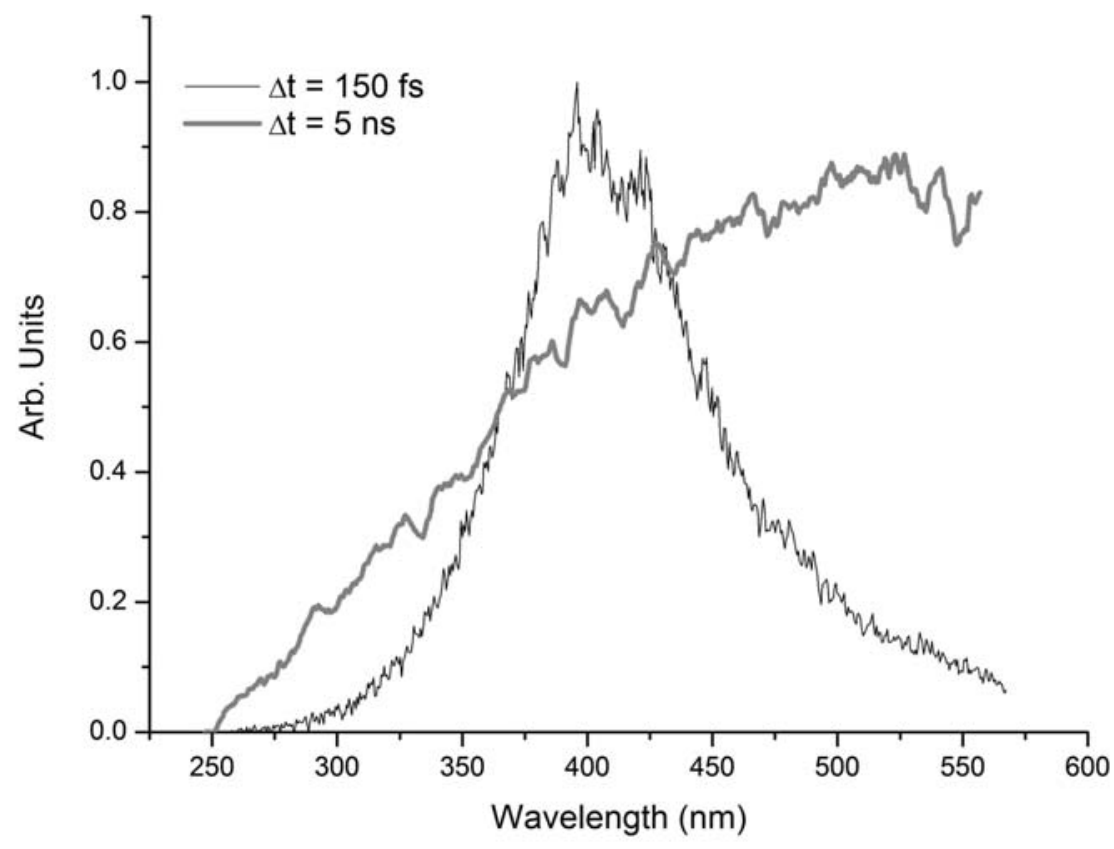

Figure 6.12

Spectra obtained from $\mathrm{SiO}_{2}$ during experiments with identical geometry, but pumped with a 1064-nm 5-ns pulse or 820-nm 150-fs pulse.

When the dynamics of the breakdown process are considered, blackbody radiation is not expected. The spectra in figure 6.12 are from two experiments performed identically, except for the radiation source. The difference in the spectra is likely due to the geometry of the ionized region. With a femtosecond pulse the ionized region is expected to be a thin disk, where as for ns pulses it is an ellipsoid. The difference in geometries results from ns pulses being long enough for a laser driven detonation wave to propagate backwards from the point of breakdown. This is not possible for the shorter duration pulses. Because the volume of plasma is so small for the fs experiments, emission from the bulk will be negligible compared to scattered laser light. 
The fact that the emission is blackbody for ns pulse excitation can be explained by taking into consideration the conditions following material breakdown and ionization $[11,18]$. Once the initial free electrons are created, they oscillate in the laser's electric field gaining thermal energy due to collisions and ionizing new atoms. The ionization rate by electron impact is given by

$$
\frac{d n_{e}}{d t}=\alpha I n_{e}
$$

where $n_{e}$ is the free electron (conduction band) density and $I$ is the laser intensity. The impact ionization coefficient for fused silica is $\alpha \sim 10 \mathrm{GW} \mathrm{cm} / \mathrm{ns}$ [11] neglecting any losses. For a dense cold plasma, $\alpha$ must have the same order of magnitude as in the surrounding fused silica. For a laser intensity of $I=120$ $\mathrm{GW} / \mathrm{cm}^{2}$ the ionization time is about 1 picosecond, three orders of magnitude less than the 5-ns duration of the 1064-nm pulses. As a result, the electron concentration during the laser pulse can easily increase up to the critical density $n_{c}$ via impact ionization, which for $1-\mu m$ laser light is $n_{e}=10^{21} \mathrm{~cm}^{-3}$. This means that only a fraction, on the order of $1 \%$ of the atoms are ionized and the plasma is strongly collisional (has a short Debye length). To evaluate the electron collision rate, both electron-ion and electron-neutral interactions must be considered. The electron-ion collision rate $\left(v_{\mathrm{ei}}\right)$ is about $10^{15} \mathrm{sec}^{-1}$. Electronneutral collision rates in fused silica could be as large as $10^{16} \mathrm{sec}^{-1}$, which is the value for cold fused silica [19]. Under these conditions, the absorption length for photons with frequencies up to the fused silica absorption edge is smaller than the size of the ionized region. Therefore, energy that would have been quickly dissipated is trapped by the plasma and the photon undergoes at least one 
collision (thus becoming thermalized) before leaving the ionized region, accounting for the observation of the Planckian spectral profile of the emission. As the inelastic collision time for electrons with the lattice ions is on the order of $10 \mathrm{ps}$, the temperature of the electrons and lattice must be the same [11]. Therefore the measured temperature evolution of the plasma also represents that of the lattice.

The electron density produced purely by thermal process is described by the Saha formula. If all the atoms are only singly ionized and the density of ions and electrons are equal then the electron density is given by equation 5.2 [20]

$$
n_{e}^{2}=6 \cdot 10^{21} N T^{3 / 2} e^{-\frac{I}{T}}
$$

where $\mathrm{N}, \mathrm{T}$, and I are the density of neutral atoms, temperature and ionization potential, respectively. To dissociate the molecules of silica from one another requires $3.6 \mathrm{eV}$ per molecule. An additional $8.9 \mathrm{eV}$ per molecule is needed to reduce the molecules to individual atoms. Finally, the energy needed to ionize the individual atoms is listed in table 6.1.

\begin{tabular}{|l|l|}
\hline Element & Ionization potential , eV \\
\hline $\mathrm{O}$ & 13.6 \\
\hline $\mathrm{Si}$ & 8.15 \\
\hline $\mathrm{K}$ & 4.34 \\
\hline $\mathrm{P}$ & 10.486 \\
\hline
\end{tabular}

Table 6.1

The energies necessary to singly ionize the relevant elements in $\mathrm{SiO}_{2}$ and DKDP.

Because blackbody radiation is observed at all delay times, the radiation must be thermalized either by high electron density or because the material has become opaque. One would expect a rapid recombination of electrons and a 
departure from thermal emission (associated with the initial high electron density) within a few picoseconds after the termination of the laser pulse due to electronion recombination. At the observed temperatures of $10^{4} \mathrm{~K}$, the electron density that would be supported thermally for $\mathrm{SiO}_{2}$ is only on the order of $10^{16}$ electrons $/ \mathrm{cm}^{-3}$, which is well below the electron density needed to support opaque plasma. Electrons in a plasma of this (relatively) low temperature and high density however, would experience substantial shielding from the ions, greatly slowing recombination, and trapping the initial radiation. This will allow radiation to appear thermal for longer delays and until the lattice has become opaque.

By performing the direct measurement of the peak temperature of the ionized region, the pressure and internal energy of the ionized region can be found from the equation of state. For the case of $\mathrm{SiO}_{2}$, the internal energy of the ionized region estimated from the equation of state is $1.6 \mathrm{mj}$ [21]. From Wien's displacement law the thermal radiation energy is peaked at photon energies of 2.8 times the temperature. For the observed temperatures (on the order of $1 \mathrm{eV}$ ) the thermal radiation is within the transparency range of the material studied. Hence, the radiation flux can be estimated as $A \sigma T^{4}$ (see figure 6.13). By integrating the radiation flux we estimate that only about $1 \%$ of the absorbed energy is dissipated by radiation. In addition, it can be estimated from the equation of state for $\mathrm{SiO}_{2}$ (figure 6.13) that a temperature of about $1 \mathrm{eV}$ corresponds to an internal energy density of $\varepsilon \sim 30 \mathrm{~kJ} / \mathrm{cm}^{3}$ and pressure of $\sim 250$ kbar. 


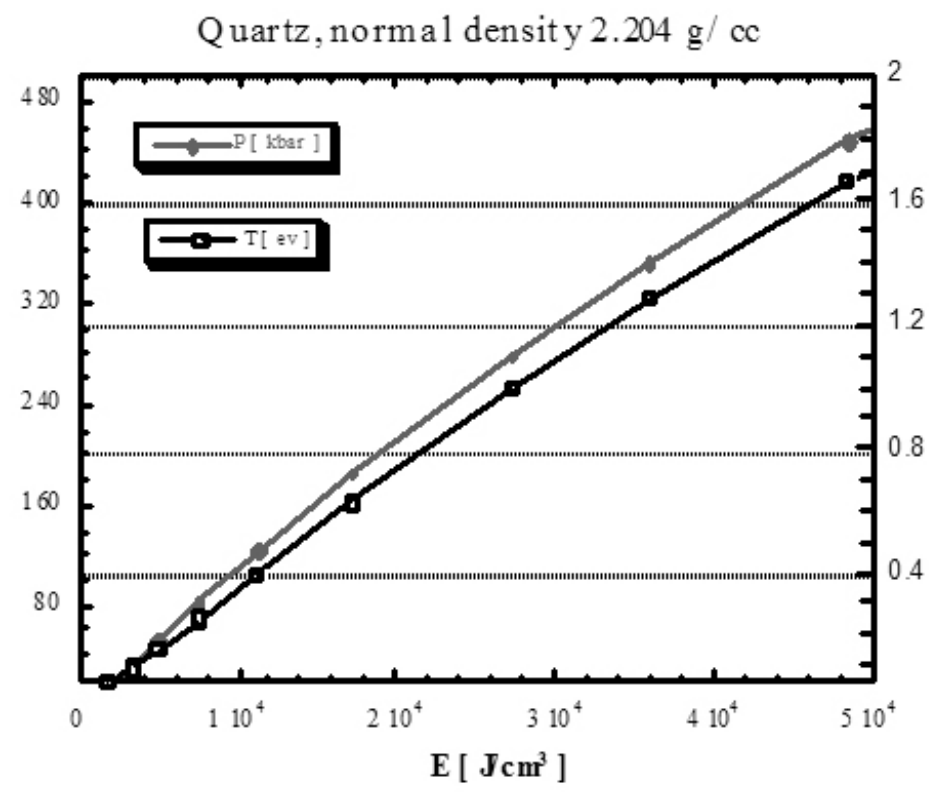

Figure 6.13

Plot by A. Rubenchik et al. from SESAME Report on the Los Alamos Equation of State Library. (1983) The equation of state enables pressure and energy density estimates from the temperature measurements.

The cooling of the observed emission must be the result of thermal conduction, radiation cooling, and release wave propagation. The energy dissipation due to thermal conduction can be estimated by subtracting the estimated losses from radiation from the change in internal energy of the plasma for delay times longer than at least $20 \mathrm{~ns}$. This is because $20 \mathrm{~ns}$ is the upper limit of the time needed for the shockwave to propagate beyond the $25 \mu \mathrm{m}$ radius of the original ionized region (typical shock waves propagate at speeds on the order of 3 to $5 \mu \mathrm{m} / \mathrm{ns}$ ). 


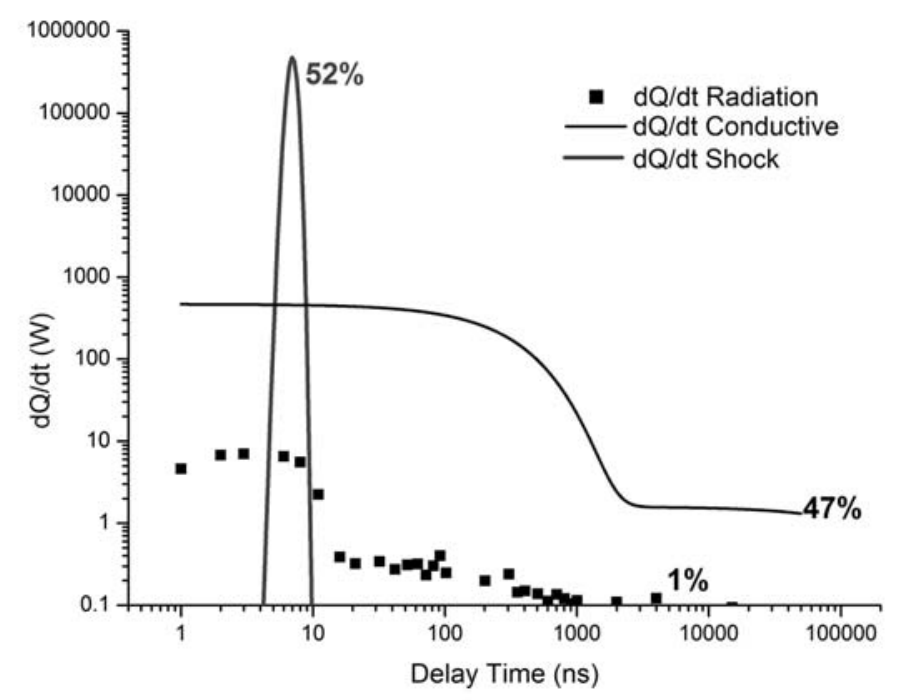

Figure 6.14

The partition of energy dissipation for the damage site.

The sharp drop in temperature observed at $\sim 10 \mathrm{~ns}$ can be assigned to energy lost to the release wave. Because the minimum integration time of the experiment is $5 \mathrm{~ns}$, the initial drop in temperature may be faster than it appears. The temporal evolution of the energy dissipation within the localized region of laser-induced damage is partitioned into each mechanism in figure 6.14.

After the initial fast temperature drop due to the release wave, the classical temperature decay rate $\left(T \sim e^{-t / \tau}\right)$ can be used as a self consistency check by comparing the projected cooling rate $(\tau)$ to that seen in figure 6.14 .

$$
\tau=\frac{\rho c a^{2}}{3 k}=\frac{a^{2}}{3 D} ; D=\frac{k}{\rho c}
$$

Where $\rho, c, a, k$, and $D$ are density, heat capacity, the radius of the ionized region, thermal conductivity, and thermal diffusivity respectively. The cooling time for a size (a) of 25 microns in radius is in the microsecond range, which is consistent with the cooling rates shown in figure 6.14. 
Previous reports that examined the plasma emission from bulk laser damage did not recognize the blackbody nature of this emission because longer temporal resolutions were used or self absorption by the material was present $[23,24]$. Temperatures in excess of $1 \mathrm{eV}$ can also be produced in the materials displayed in table 1 by mechanical shocks [22-26]. For example, Csl when shocked to a pressure of $92.2 \mathrm{GPa}$, radiates at a temperature of $10800 \mathrm{~K}$ [24]. Temperatures as high as $1.5 \mathrm{eV}$ have also been observed during single laserinduced bubble cavitation experiments in water [27]. Because of these observations, we consider the possibility that the blackbody radiation observed during laser damage is the result of a shock wave propagating outward from sites at which absorbed laser radiation has produced GPa range pressures. Except when a change of phase is present, the temperature produced by such a shock wave will be proportional to pressure. Unlike in classical shock measurement experiments that are quasi-one dimensional, the pressure and temperature at the front (which is expanding in three dimensions) must be inversely proportional to $t^{3}$ (where $t$ is the time since the laser pulse) [23]. Since the blackbody radiation persists for hundreds of ns, shock wave propagation cannot be the predominate source of radiation. This does not preclude significant amounts of mechanical energy being dissipated by the expanding shock front. 


\subsection{Conclusions}

Laser-induced damage in optical materials may be discussed in terms of three distinct phases: damage initiation, energy absorption, and energy dissipation. In DKDP the mechanism for damage initiation is defect assisted multi-photon absorption. Electrons are promoted from the valence to conduction bands until an opaque plasma is formed. Energy is then rapidly absorbed for the duration of the laser pulse.

In all the materials studied, energy is dissipated by three mechanisms: rarefaction wave propagation, conduction, and radiation. The rarefaction wave propagating out from the damage initiation site and classical thermal conduction each account for approximately half of the energy dissipated. The flash of light accompanying the laser-induced damage radiates no more than $1 \%$ of the absorbed energy and indicates peak temperatures on the order of $1 \mathrm{eV}$. 


\subsection{References}

[1] Liu, C.S., et al., Phys Rev Lett 9101, 5505 (2003).

[2] Bloembergen, N., IEEE J. Quantum Electron. (USA) QE-10, 375-86 (1974).

[3] Jones, S.C., et al., Optical Engineering 28, 1039-1068 (1989).

[4] Feit, M.D., SPIE, 1997. 3244: p. 487.

[5] Feit, M.D.,Personal Communication "Gray particle wavelength response" (2003)

[6] Yoshida, H., et al., Applied Physics B-Lasers and Optics 70, 195-201 (2000).

[7] Runkel, M., et al., 1999: p. 374-85.

[8] Rainer, F., et al., SPIE, 1992. 1848: p. 46.

[9] Oconnell, R.M., Appl. Opt. (USA) 31, 4143-4153 (1992).

[10] Du, D., et al., Appl Phys Lett 64, 3071-3073 (1994).

[11] Stuart, B.C., et al., Phys. Rev. B, Condens. Matter (USA) 53, 1749-61 (1996).

[12] Natoli, J., et al., SPIE, 2002. 4932: p. 224-36.

[13] Runkel, M., et al., SPIE, 2001. 4679: p. 408-19.

[14] Swain, J., et al., Appl Phys Lett 40, 350-2 (1982).

[15] Shen, X.A., et al., Phys Rev Lett 62, 2711-13 (1989).

[16] Demos, S.G., et al., Optics Letters 26, 1975-7 (2001).

[17] Demos, S.G., et al., 1999: p. 509-15.

[18] Carr, C.W., et al., Physical Review Letters (2003).

[19] Arnold, D., et al., Physical Review B Condensed Matter 45, 1477-80 (1992).

[20] Chen, F.F., Introduction to plasma physics and controlled fusion (:Plenum Press, New York, 1984).

[21] T4_Group, SESAME Report on the Los Alamos Equation of State Library. 1983: Los Alamos.

[22] Radousky, H.B., et al., Phys Rev Lett 57, 2419-22 (1986).

[23] Radousky, H.B., et al., Review of Scientific Instruments 60, 3707-10 (1989).

[24] Radousky, H.B., et al., Physical Review B Condensed Matter 31, 1457-62 (1985).

[25] Lyzenga, G.A., et al., Journal of Geophysical Research 88, 2431-44 (1983).

[26] Schmitt, D.R., et al., Journal of Geophysical Research 94, 5851-71 (1989).

[27] Baghdassarian, O., et al., Phys Rev Lett 86, 4934-4937 (2001). 